Prepared in cooperation with the Mojave Water Agency

\title{
Preliminary Hydrogeologic Assessment near the Boundary of the Antelope Valley and El Mirage Valley Groundwater Basins, California
}

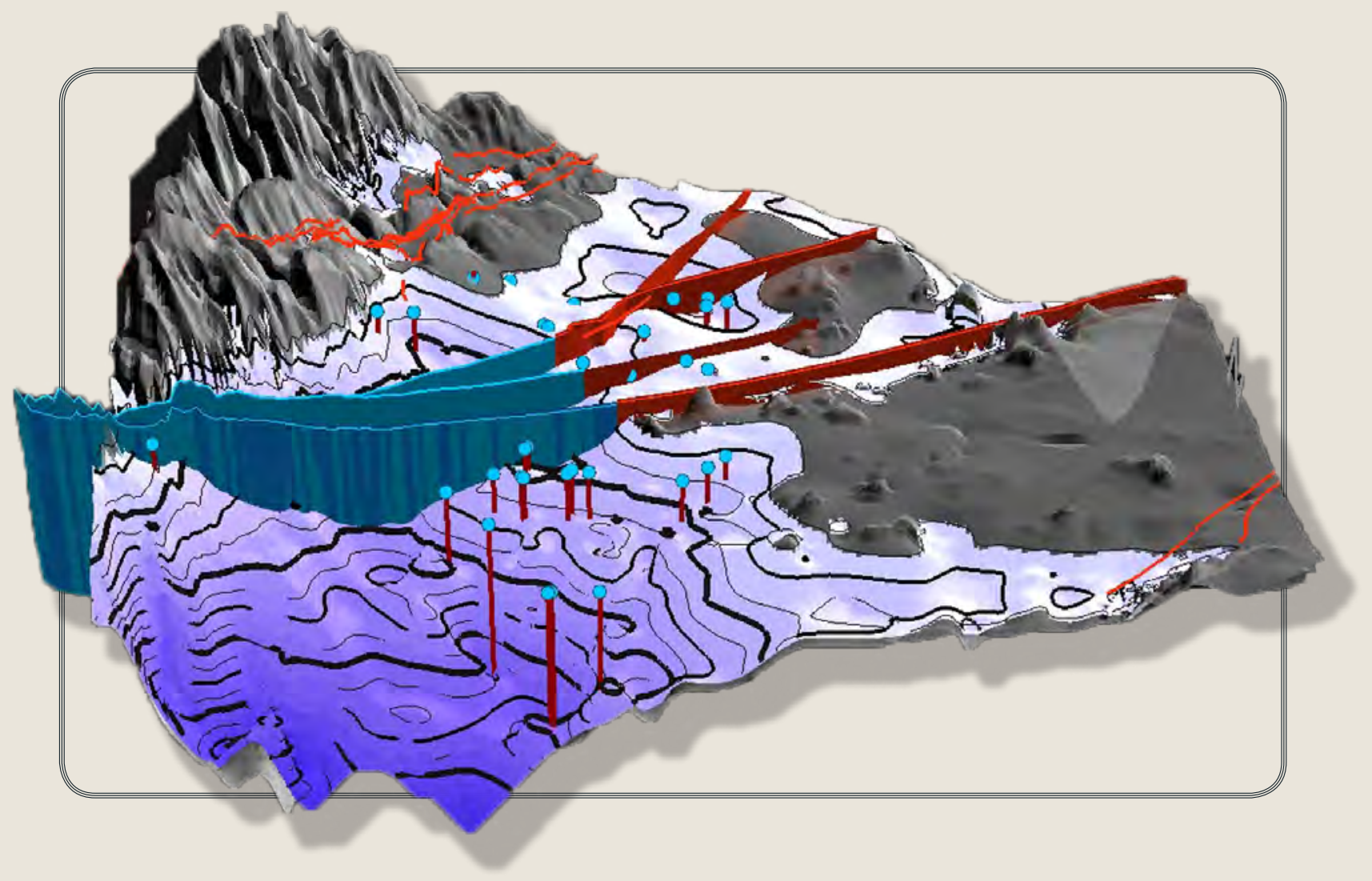

Scientific Investigations Report 2017-5065 
Cover. Visualization of the saturated alluvium, faults, and subsurface basement topography of the Antelope Valley and El Mirage Valley groundwater basins, California. Visualization by Donald J. Martin and Sally F. House. 


\section{Preliminary Hydrogeologic Assessment near the Boundary of the Antelope Valley and El Mirage Valley Groundwater Basins, California}

By Christina L. Stamos, Allen H. Christensen, and Victoria E. Langenheim

Prepared in cooperation with the Mojave Water Agency

Scientific Investigations Report 2017-5065 


\title{
U.S. Department of the Interior \\ RYAN K. ZINKE, Secretary
}

\section{U.S. Geological Survey William H. Werkheiser, Acting Director}

\author{
U.S. Geological Survey, Reston, Virginia: 2017
}

For more information on the USGS - the Federal source for science about the Earth, its natural and living resources, natural hazards, and the environment-visit https://www.usgs.gov or call 1-888-ASK-USGS.

For an overview of USGS information products, including maps, imagery, and publications, visit https://www.usgs.gov/pubprod/.

Any use of trade, firm, or product names is for descriptive purposes only and does not imply endorsement by the U.S. Government.

Although this information product, for the most part, is in the public domain, it also may contain copyrighted materials as noted in the text. Permission to reproduce copyrighted items must be secured from the copyright owner.

Suggested citation:

Stamos, C.L., Christensen, A.H., and Langenheim, V.E., 2017, Preliminary hydrogeologic assessment near the boundary of the Antelope Valley and El Mirage Valley groundwater basins, California: U.S. Geological Survey Scientific Investigations Report 2017-5065, 44 p., https://doi.org/10.3133/sir20175065.

ISSN 2328-0328 (online) 


\section{Acknowledgments}

This study was completed in cooperation with the Mojave Water Agency. Their support and provision of resources were invaluable for the completion of the study. Appreciation is also extended to the staff of the Phelan-Piñon Hills Community Services District for access to their data and assistance in the field.

Further appreciation is extended to our U.S. Geological Survey colleagues Joseph M. Nawikas and David B. Dillon for their efforts in collecting the groundwater-level data from the field, to Andrew Y. Morita for collecting the gravity data, and to Sally F. House and Donald J. Martin for creating the visualizations used in this report. 


\section{Contents}

Abstract
Introduction
Purpose and Scope
Description of the Study Area
Pydrogeologic Setting
Hydrologic System
Recharge




\section{Figures}

1. Map showing location of study area and boundaries of the groundwater basins near Piñon Hills, California...

2. Map showing generalized geologic setting of the study area, near Piñon Hills, California

3. Map showing regional basin gravity field near Piñon Hills, California ..............................8

4. Map showing location of gravity stations for the regional study and gravity stations and isostatic residual gravity field for this 2014 study, near Piñon Hills, California

5. Map showing estimated thickness of basin-fill deposits based on modeled gravity field, near Piñon Hills, California.

6. Map showing approximate altitude of basement complex from modeled gravity data and location of hydrogeologic sections and wells on section shown in figure 12, near Piñon Hills, California

7. Map showing location of wells with groundwater-level measurements available in 2014, for 1917-2014, near Piñon Hills, California ...

8. Graph showing number of wells with recorded groundwater-level measurements, 1917-2014, near Piñon Hills, California.

9. Map showing potential water-level measurement well sites visited during September 2014, near Piñon Hills, California

10. Map showing groundwater-level altitude, general direction of groundwater flow (March 2014-March 2015), and location of wells with groundwater-level hydrographs shown in figure 11, near Piñon Hills, California

11. Hydrographs showing groundwater level in wells. $A, 1980-2015$, and B, 1950-2015 and 1915-2015, near Piñon Hills, California

12. Generalized hydrogeologic cross sections showing groundwater-level altitude in 2014-15 and estimated altitude of the basement complex from the modeled gravity data: $A$, west to east $\left(A-A^{\prime}\right) ; B$, south to north $(B-B)$; and $C$, south to north $(C-C)$, near Piñon Hills, California.

13. Animation showing the altitude of the top of the basement rocks based on the gravity data and altitude of the water table in 2014-15, near Piñon Hills, California ......38

14. Animation showing the potential dewatering of the saturated alluvium starting with the 2014-15 water-table altitude and assuming an incremental 16.4 feet (5 meter) drop per frame of the water table, near Piñon Hills, California

\section{Tables}

1. Gravity measurements collected in 2014 near Piñon Hills, California

2. Data for wells with depth information used to constrain the gravity model near Piñon Hills, California .

3. Estimated density contrast varied with depth near Piñon Hills, California

4. Selected wells with groundwater-level data, 1990-2015, near Piñon Hills, California ...29 


\section{Conversion Factors}

U.S. customary units to International System of Units

\begin{tabular}{|c|c|c|}
\hline Multiply & By & To obtain \\
\hline \multicolumn{3}{|c|}{ Length } \\
\hline inch (in.) & 2.54 & centimeter $(\mathrm{cm})$ \\
\hline foot $(\mathrm{ft})$ & 0.3048 & meter $(\mathrm{m})$ \\
\hline mile (mi) & 1.609 & kilometer (km) \\
\hline \multicolumn{3}{|c|}{ Area } \\
\hline acre & 4,047 & square meter $\left(\mathrm{m}^{2}\right)$ \\
\hline acre & 0.4047 & hectare (ha) \\
\hline square mile $\left(\mathrm{mi}^{2}\right)$ & 2.590 & square kilometer $\left(\mathrm{km}^{2}\right)$ \\
\hline \multicolumn{3}{|c|}{ Volume } \\
\hline acre-foot (acre-ft) & 1,233 & cubic meter $\left(\mathrm{m}^{3}\right)$ \\
\hline \multicolumn{3}{|c|}{ Flow rate } \\
\hline acre-foot per year (acre-ft/yr) & 1,233 & cubic meter per year $\left(\mathrm{m}^{3} / \mathrm{yr}\right)$ \\
\hline foot per year ( $\mathrm{ft} / \mathrm{yr}$ ) & 0.3048 & meter per year $(\mathrm{m} / \mathrm{yr})$ \\
\hline inch per year (in/yr) & 25.4 & millimeter per year (mm/yr) \\
\hline \multicolumn{3}{|c|}{ Density } \\
\hline pound per cubic foot $\left(\mathrm{lb} / \mathrm{ft}^{3}\right)$ & 16.02 & kilogram per cubic meter $\left(\mathrm{kg} / \mathrm{m}^{3}\right)$ \\
\hline \multicolumn{3}{|c|}{ Gravity (acceleration) } \\
\hline feet per square second $\left(\mathrm{ft} / \mathrm{s}^{2}\right)$ & 0.00003 & milligal (mGal) \\
\hline
\end{tabular}

Temperature in degrees Celsius $\left({ }^{\circ} \mathrm{C}\right)$ may be converted to degrees Fahrenheit $\left({ }^{\circ} \mathrm{F}\right)$ as follows:

$$
{ }^{\circ} \mathrm{F}=\left(1.8 \times{ }^{\circ} \mathrm{C}\right)+32 .
$$

Temperature in degrees Fahrenheit $\left({ }^{\circ} \mathrm{F}\right)$ may be converted to degrees Celsius $\left({ }^{\circ} \mathrm{C}\right)$ as follows:

$$
{ }^{\circ} \mathrm{C}=\left({ }^{\circ} \mathrm{F}-32\right) / 1.8 \text {. }
$$

\section{Datum}

Vertical coordinate information is referenced to the North American Vertical Datum of 1988 (NAVD 88).

Horizontal coordinate information is referenced to the North American Datum of 1983 (NAD 83). Altitude, as used in this report, refers to distance above the vertical datum. 


\section{Abbreviations}

$\begin{array}{ll}\text { 3-D } & \text { three-dimensional } \\ \text { BCM } & \text { Basin Characterization Model } \\ \text { D-79 } & \text { LaCoste and Romberg D-meter } \\ \text { DWR } & \text { California Department of Water Resources } \\ \text { GPS } & \text { Global Positioning System } \\ \text { MWA } & \text { Mojave Water Agency } \\ \text { NWISWeb } & \text { National Water Information System web page } \\ \text { OPUS } & \text { National Geodetic Survey Online Positioning User Service } \\ \text { RTK } & \text { Trimble Real-Time Kinematic } \\ \text { SGMA } & \text { Sustainable Groundwater Management Act } \\ \text { SR-18 } & \text { California State Route 18 } \\ \text { SR-138 } & \text { California State Route 138 } \\ \text { TEM } & \text { time-domain electromagnetic } \\ \text { USGS } & \text { U.S. Geological Survey }\end{array}$

\section{Well-Numbering System}

Wells are identified and numbered according to their location in the rectangular system for the subdivision of public lands. Identification consists of the township number, north or south; the range number, east or west; and the section number. Each section is divided into 1640 -acre tracts lettered consecutively (except " $I$ " and " 0 "), beginning with " $A$ " in the northeast corner of the section and progressing in a sinusoidal manner to " $R$ " in the southeast corner. Within the 40 -acre tract, wells are sequentially numbered in the order they are inventoried. The final letter refers to the base line and meridian. In California, there are three base lines and meridians: Humboldt (H), Mount Diablo (M), and San Bernardino (S). All wells in the study area are referenced to the San Bernardino base line and meridian (S). Well numbers consist of 15 characters and follow the format 005N008W13R001S. In this report, well numbers are abbreviated and written 5N/8W-13R1. Wells in the same township and range are referred to only by their section designation, 13R1. The following diagram shows how the number for well $5 \mathrm{~N} / 8 \mathrm{~W}-13 \mathrm{R} 1$ is derived.

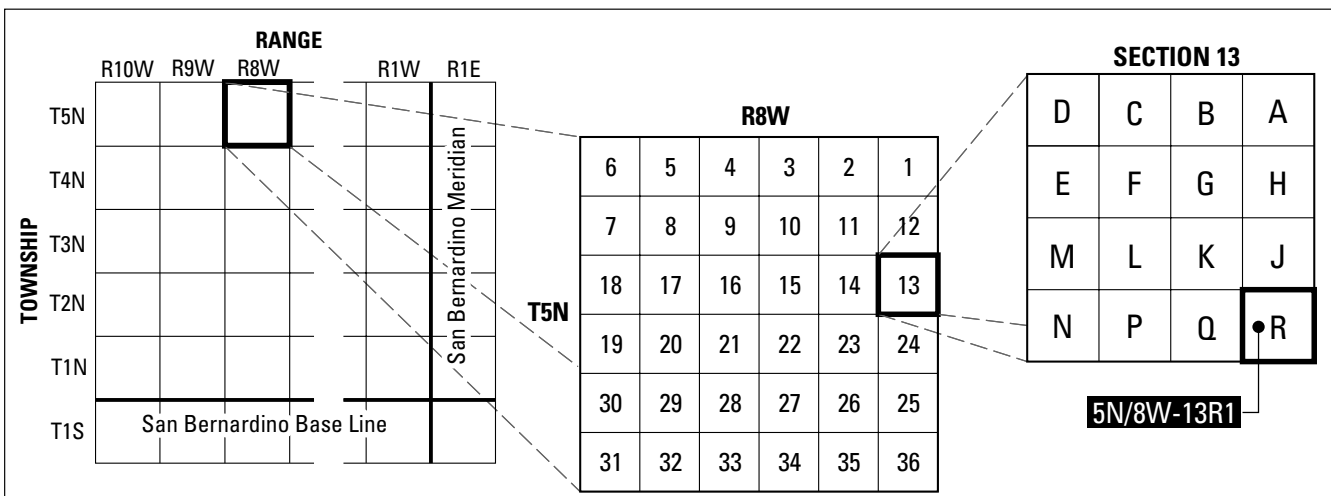

Well-numbering diagram (Note: maps in this report use abbreviated well numbers such as 13R1) 



\title{
Preliminary Hydrogeologic Assessment near the Boundary of the Antelope Valley and El Mirage Valley Groundwater Basins, California
}

\author{
By Christina L. Stamos, Allen H. Christensen, and Victoria E. Langenheim
}

\section{Abstract}

The increasing demands on groundwater for water supply in desert areas in California and the western United States have resulted in the need to better understand groundwater sources, availability, and sustainability. This is true for a 650-square-mile area that encompasses the Antelope Valley, El Mirage Valley, and Upper Mojave River Valley groundwater basins, about 50 miles northeast of Los Angeles, California, in the western part of the Mojave Desert. These basins have been adjudicated to ensure that groundwater rights are allocated according to legal judgments. In an effort to assess if the boundary between the Antelope Valley and El Mirage Valley groundwater basins could be better defined, the U.S. Geological Survey began a cooperative study in 2014 with the Mojave Water Agency to better understand the hydrogeology in the area and investigate potential controls on groundwater flow and availability, including basement topography.

Recharge is sporadic and primarily from small ephemeral washes and streams that originate in the San Gabriel Mountains to the south; estimates range from about 400 to 1,940 acre-feet per year. Lateral underflow from adjacent basins has been considered minor in previous studies; underflow from the Antelope Valley to the El Mirage Valley groundwater basin has been estimated to be between 100 and 1,900 acre-feet per year. Groundwater discharge is primarily from pumping, mostly by municipal supply wells. Between October 2013 and September 2014, the municipal pumpage in the Antelope Valley and El Mirage Valley groundwater basins was reported to be about 800 and 2,080 acre-feet, respectively.

This study was motivated by the results from a previously completed regional gravity study, which suggested a northeasttrending subsurface basement ridge and saddle approximately 3.5 miles west of the boundary between the Antelope Valley and El Mirage Valley groundwater basins that might influence groundwater flow. To better define potential basement structures that could affect groundwater flow between the groundwater basins in the study area, gravity data were collected using more closely spaced measurements in September 2014. Groundwater-level data was gathered and collected from March 2014 through March 2015 to determine depth to water and direction of groundwater flow. The gravity and groundwater-level data showed that the saturated thickness of the alluvium was about 2,000 feet thick to the east and about 130 feet thick above the northward-trending basement ridge near Llano, California. Although it was uncertain whether the basement ridge affects the groundwater system, a potential barrier to groundwater flow could be created if the water table fell below the altitude of the basement ridge, effectively causing the area to the west of the basement ridge to become hydraulically isolated from the area to the east. In addition, the direction of regional-groundwater flow likely will be influenced by future changes in the number and distribution of pumping wells and the thickness of the saturated alluvium from which water is withdrawn. Three-dimensional animations were created to help visualize the relation between the basins' basement topography and the groundwater system in the area. Further studies that could help to more accurately define the basins and evaluate the groundwater-flow system include exploratory drilling of multi-depth monitoring wells; collection of depth-dependent water-quality samples; and linking together existing, but separate, groundwater-flow models from the Antelope Valley and El Mirage Valley groundwater basins into a single, calibrated groundwater-flow model.

\section{Introduction}

The limited availability of surface-water resources in many parts of the Mojave Desert (fig. 1) has resulted in the reliance on groundwater for agricultural, public, and domestic water supply. The rapid expansion of agriculture and population since the mid-1950s, along with reliance on groundwater for water supply, have resulted in the need to better understand groundwater availability and explore methods for ensuring its sustainability. The demands for this limited resource by separate entities have led to complex water-rights decisions and the need to better document groundwater use. About two dozen groundwater basins in California, mostly in the southern part of the state, have been adjudicated to ensure that groundwater rights are allocated according to legal judgments (California Department of Water Resources, 2016). 


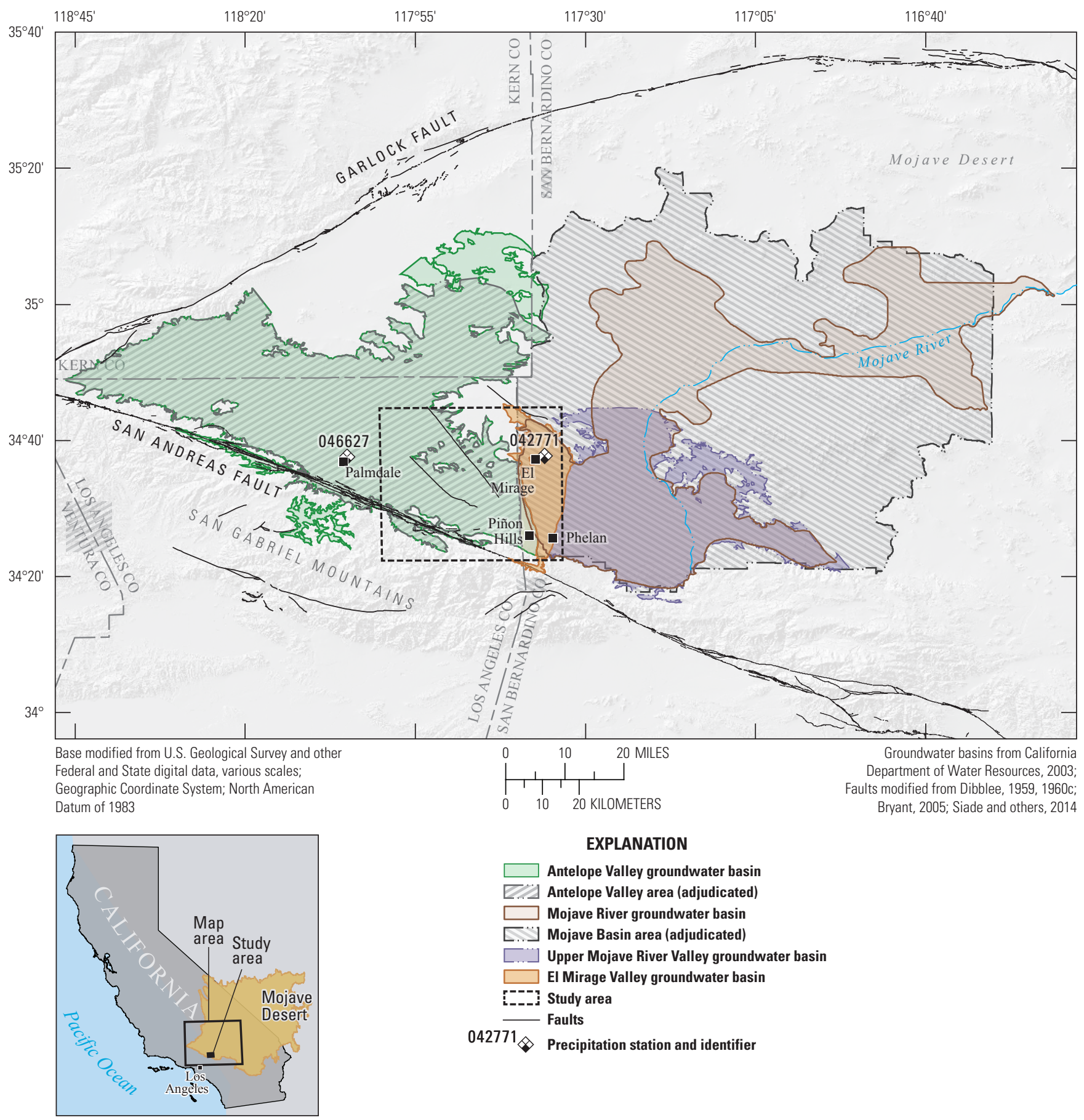

Figure 1. Location of study area and boundaries of the groundwater basins near Piñon Hills, California. 
The study area includes parts of three groundwater basins defined by the California Department of Water Resources (DWR; California Department of Water Resources, 2003): Antelope Valley, El Mirage Valley, and Upper Mojave River Valley groundwater basins (fig. 1). The Antelope Valley and El Mirage Valley groundwater basins share a common boundary for about 15 miles (mi) slightly east of the Los Angeles and San Bernardino County line. Previous hydrologic studies (Izbicki and others, 1995; Stamos and Predmore, 1995; Stamos and others, 2001) have modified the boundaries of El Mirage Valley, Upper Mojave River Valley, and five other groundwater basins defined by the DWR (California Department of Water Resources, 2003) and have collectively referred to them as the Mojave River groundwater basin (fig. 1).

The Mojave Basin Area was adjudicated in 1993, and the Mojave Water Agency (MWA) was appointed as Watermaster to ensure that water rights are allocated according to the Court Judgment (Riverside County Superior Court, 1996). As Watermaster, the MWA is responsible for monitoring and verifying water production, collecting required assessments, conducting studies, and preparing an annual report of its findings and activities for the Mojave Basin Area to the Superior Court of California, County of Riverside.

In November 2015, the Superior Court of California, County of Los Angeles, issued its tentative decision on the Antelope Valley Groundwater case, clearing the way for water resource management and a comprehensive adjudication of the Antelope Valley groundwater basin's groundwater rights for a judgment, or "physical solution" (Superior Court of the State of California, 2015). Similar to the MWA, the prospective Watermaster for the Antelope Valley adjudicated area will be responsible for the fair and equitable administration of the Court Judgment and to assess parties who exceed pumping limits to help pay for replacement water from the California State Water Project (California Department of Water Resources, 2015a). The Antelope Valley and Mojave Basin adjudicated areas do not coincide with the groundwater basins defined by the DWR (2003), nor do they encompass the groundwater basins in their entirety (fig. 1).

Interest in understanding the hydrogeology in the area has been heightened by passage of California's Sustainable Groundwater Management Act (SGMA) in 2014 (California Department of Water Resources, 2015b). The SGMA established a framework of priorities and requirements to help local agencies sustainably manage groundwater within a basin, or subbasin, and allows local agencies to customize groundwater sustainability plans to their regional economic and environmental needs. The SGMA empowers local agencies to manage groundwater basins by collecting and analyzing data to better understand and sustainably manage their water resources.

The two separate court judgments and passage of the SGMA have heightened interest in understanding groundwater movement near the shared boundary of the adjudicated Antelope Valley groundwater basin and the Mojave Basin Area (fig. 1). In an effort to assess the potential exchange of groundwater between the basins, the U.S. Geological Survey
(USGS) began a cooperative study in 2014 with the MWA with the purpose of determining if the characteristics of the hydrologic boundary between the Antelope Valley and El Mirage Valley groundwater basins could be better defined or determined. Geophysical, geological, and hydrological data near the boundary are sparse, and coordinated, in-depth hydrogeologic studies have not previously characterized the groundwater system in the vicinity of the boundary.

The objectives of this study were to determine (1) the direction of groundwater flow in the boundary region between the two groundwater basins, (2) the thickness of the unconsolidated deposits and depth to consolidated rocks of the basement complex in the area using surface-geophysical gravity techniques used in this and previous studies, and (3) controls or influences on groundwater flow exerted by the concealed basement topography.

\section{Purpose and Scope}

The purpose of this report is to document the results of a preliminary assessment of the hydrogeology using (1) the collection and interpretation of a surface-geophysical gravity survey, (2) the compilation of available well information, and (3) a field reconnaissance survey of existing wells and the collection of groundwater levels to determine depth to groundwater. The gravity and groundwater-level data gathered for this study were collected from March 2014 through March 2015. The area for which the groundwater-level data were collected and the extent of the available regional gravity data that were used for this study are shown in figure 2. Results from the data collected were used to derive and visualize an estimation of the saturated alluvial thickness and the configuration of the basement topography.

\section{Description of the Study Area}

The study area covers about 650 square miles $\left(\mathrm{mi}^{2}\right)$ in the western part of the Mojave Desert, on the northern side of the San Gabriel Mountains, about $50 \mathrm{mi}$ northeast of Los Angeles, California (figs. 1, 2). It is typical of desert alluvial-filled basins that are bounded by barren hills, ridges, and buttes. The study area straddles the Los Angeles-San Bernardino County line; the western part of the study area lies within the Antelope Valley groundwater basin, and the eastern part includes the El Mirage Valley and Upper Mojave River Valley groundwater basins. From south to north, the land-surface altitude slopes from the base of the San Gabriel Mountains and San Andreas Fault, about 6,000 feet (ft) above the North American Vertical Datum of 1988 (NAVD 88), to an altitude of less than 2,400 ft at the northwestern edge of the study area. There are no perennial stream channels, but several well-developed ephemeral stream channels exist in the study area: Big Rock Wash, Mescal Creek, and Le Montaine Creek in the Antelope Valley groundwater basin, and Sheep Creek Wash in the El Mirage Valley groundwater basin (fig. 2). These ephemeral channels 


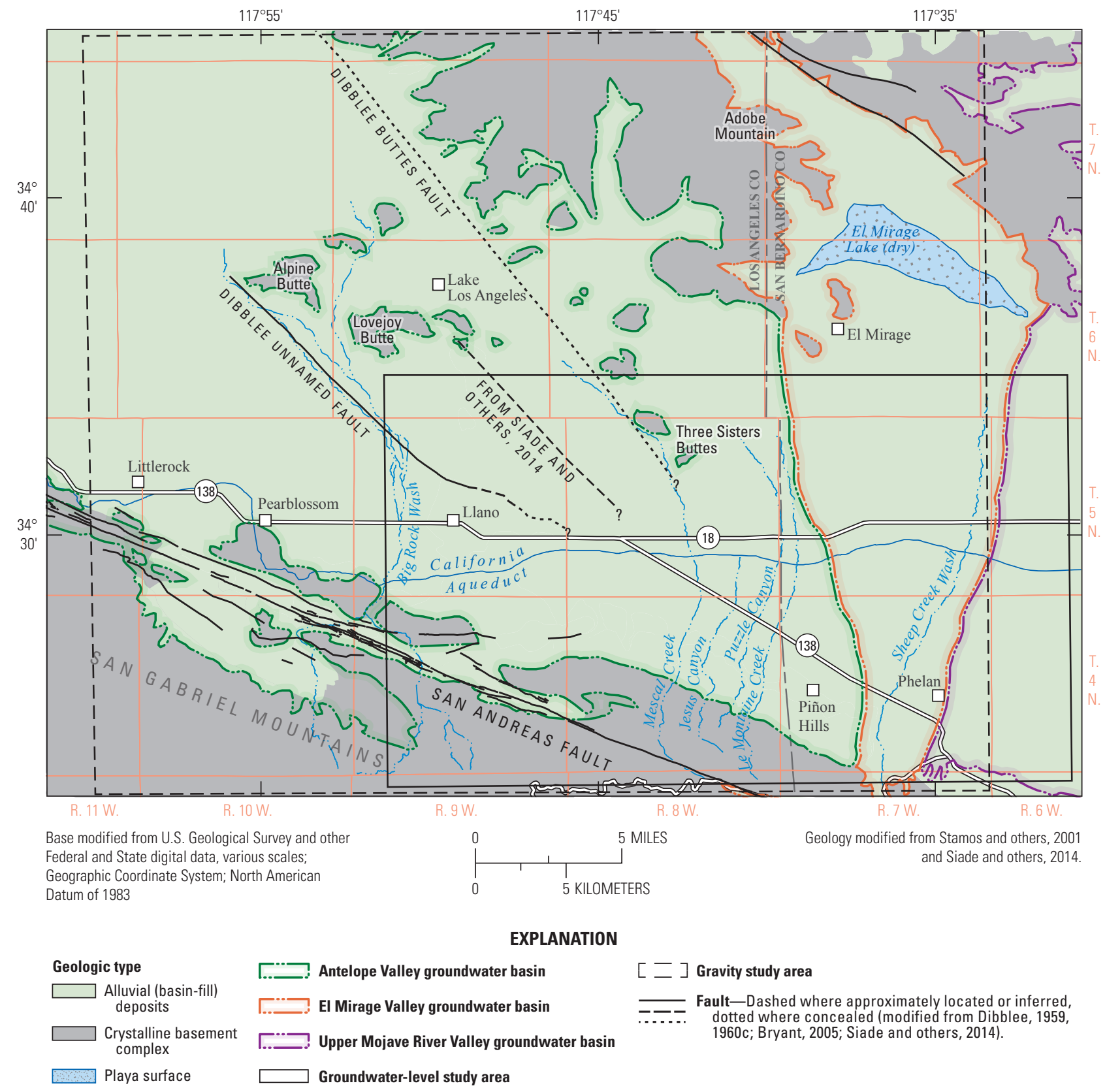

Figure 2. Generalized geologic setting of the study area, near Piñon Hills, California. 
convey runoff only in response to snow melt, flash floods, and heavy precipitation, usually from storms in the mountains (Marston and Marston, 2017). El Mirage Lake (dry) is a playa (fig. 2), that is, a topographic low where ponding of excess surface water from heavy precipitation or large ephemeral flows that do not infiltrate along Sheep Creek Wash typically persists only for a few weeks per year.

The climate of the area is typical of arid desert environments: high summer temperatures, high evaporation rates, and minimal precipitation. Humidity is low, and temperatures frequently exceed $100^{\circ} \mathrm{F}$ in the summer and can drop below freezing in the winter. The average annual precipitation measured at local weather stations was 5.05 inches (in.) at Palmdale, Calif. (1934-2015), and 5.81 in. at El Mirage, Calif. (1971-2010; Western Regional Climate Center, 2015a, 2015b; fig. 1). Most precipitation occurs between October and March, with occasional flash flooding from brief monsoonal thunderstorms during the summer months.

The study area contains widely dispersed rural populations and small towns; the nearest urban city is Palmdale, Calif., which is about $25 \mathrm{mi}$ to the west of the boundary between the groundwater basins and had an estimated population of about 158,000 in 2015 (U.S. Census Bureau, 2017). The largest community in the study area is Phelan, Calif., which had a population of about 14,000 in 2010 (U.S. Census Bureau, 2017).

Groundwater pumping for domestic supplies likely has been minimal, although it has never been quantified. Depth to water in the study area varies from less than $100 \mathrm{ft}$ in the northwestern part of the study area to greater than $600 \mathrm{ft}$ below land surface north of Piñon Hills, Calif. (Siade and others, 2014; fig. 2). Agricultural pumping occurs in a few places along the northern boundary of the study area, and pumping for municipal supply from deep production wells along the Los Angeles-San Bernardino County line is the main source of water for local communities. The California Aqueduct bisects the study area (fig. 2); no deliveries from the cement-lined aqueduct are made to the study area.

\section{Hydrogeologic Setting}

The area of the Mojave Desert comprising the Antelope Valley, El Mirage Valley, and Upper Mojave River Valley groundwater basins contains large, alluvial-filled structural depressions that are downfaulted between the Garlock and San Andreas Fault Zones (Dibblee, 1967; fig. 1). The basinfill deposits consist of locally derived Quaternary and Tertiary sediments, which overlay pre-Cenozoic metamorphic and igneous (crystalline) rocks that form the basement complex. The underlying basement complex, which is not considered water bearing, forms the base and margins of the groundwater basins and crops out in the highlands within the study area. This basement complex consists of pre-Cenozoic igneous and metamorphic rocks (Hewett, 1954; Dibblee, 1963) that crop out to the southeastern part of the study area in or near the San Gabriel Mountains (Stamos and others, 2001) and form inselbergs, such as Three Sisters Buttes (fig. 2).

The water-bearing, basin-fill deposits that compose the groundwater system overlying the basement complex are unconsolidated alluvial and fan deposits of Quaternary age and semi-consolidated to consolidated deposits of Tertiary age (Dutcher and Worts, 1963). The deeper sediments of interfingering older alluvium and older fan deposits typically are more compacted and indurated than the shallower sediments of the younger alluvium and younger fan deposits. Alluvial deposits in parts of the Antelope Valley groundwater basin west of Palmdale, Calif. (fig. 1), were estimated to be as much as $5,000 \mathrm{ft}$ thick by Dutcher and Worts (1963) and more than $10,000 \mathrm{ft}$ thick by Jachens and others (2014). Jachens and others (2014) estimated that the alluvial deposits were much thinner in the study area, about 2,000 ft thick, but the estimate was based on coarsely spaced data, resulting in larger uncertainties in the estimates of the thickness of the alluvial deposits. Runoff from the northern slopes of the San Gabriel Mountains recharges the groundwater system along the mountain front and by infiltration of runoff in local creeks.

Numerous faults traverse the Antelope Valley, El Mirage Valley, and Upper Mojave River Valley groundwater basins; the reader is referred to the following publications for detailed discussions on their locations and extents: Dibblee (1959, 1960a, 1960b, 1960c, 1963), Mabey (1960), Dutcher and Worts (1963), Jennings and others (1977), Dokka and Travis (1990), Ward and others (1993), and Bryant (2005). In the southern part of the study area, the tectonic activity associated with the San Andreas Fault Zone has had the most influence on the geologic structure, climate, and hydrologic setting because transpression, or contraction, across the fault has caused uplift of the San Gabriel Mountains (fig. 1) during the past several million years (Meisling and Weldon, 1989; Matti and Morton, 1993). A complex pattern of deeply incised, redirected, and truncated stream channels has formed on the north side of the San Gabriel Mountains as result of movement along the San Andreas Fault Zone. A few unnamed northwest-trending faults have been mapped in the alluvium within the study area (Dibblee, 1959, 1960a, 1960b, 1960c; Bryant, 2005; Siade and others, 2014; fig. 2), but their relation and connectivity to the San Andreas Fault are uncertain, and their depth and effects on groundwater flow are unknown. A regional gravity survey and geologic mapping completed by Mabey (1960) indicated the presence of faults beneath the alluvial deposits and many west-northwest striking faults that are parallel to the San Andreas Fault Zone. Bloyd (1967) noted large differences in groundwater altitude along the southern boundary of the Antelope Valley groundwater basin within a few miles of the San Andreas Fault Zone. Using groundwater-level data, Bloyd (1967) also mapped faults within the Antelope Valley that are subparallel to the main segment of the San Andreas Fault Zone (fig. 2). 


\section{Previous Hydrogeologic Studies}

Geophysical, geological, and hydrological data for the study area are sparse, and no widely documented hydrogeologic study has previously defined the location of a potential hydrologic boundary or the characteristics of the groundwater system in the area where the adjudicated groundwater basins of the Antelope Valley and the Mojave Basin Area meet. Previous studies in these two groundwater basins include geologic reconnaissance (Dibblee, 1960a, 1960b), field hydrogeologic surveys and reports (Johnson, 1911; Thompson, 1929; California Department of Water Resources, 1966, 1967; Carlson and others, 1998; Teague and others, 2014), and subsurface geophysical investigations (Subsurface Surveys, Inc., 1990). Several regional groundwater-flow model studies have been conducted separately in the groundwater basins, including models developed by Durbin (1978), Leighton and Phillips (2003), and Siade and others (2014) in the Antelope Valley groundwater basin; Hardt (1971) and Stamos and others (2001) have published models within the Mojave Basin Area. In these modeling studies, the model boundaries were approximately defined by the separate groundwater basins (fig. 1), did not share a common boundary, and had different model discretization and boundary types. For example, Leighton and Phillips (2003) simulated the boundary as a no-flow type; Hardt (1971) did not extend the model to include the El Mirage Valley groundwater basin. In the two most recent groundwater models, Siade and others (2014) simulated the southeastern boundary of the Antelope Valley groundwater basin as a general-head boundary located slightly east of the San Bernardino-Los Angeles County line to address any potential exchange of groundwater into the El Mirage Valley groundwater basin (fig. 2); Stamos and others (2001) simulated the boundary as a no-flow type.

\section{Hydrologic System}

The water-bearing deposits in the study area generally are described as unconsolidated alluvial deposits of Quaternary age, which consist of sediments that vary in size from fine silts and clays to coarse sands and gravels, depending on location and depositional history. These deposits were described by Siade and others (2014) as "alluvial deposits" and by Stamos and others (2001) as "undifferentiated alluvium." The shallower deposits are generally less consolidated, have higher saturated hydraulic conductivities, and yield water to wells more freely than the deeper deposits, which are more consolidated, sometimes highly indurated, and more cemented (Dutcher and Worts, 1963; Stamos and others, 2001). No evidence of confined aquifer conditions was found for the study area; therefore, the aquifer system is assumed to be unconfined. For more detailed discussions of the individual units of these unconfined aquifer systems, the reader is referred to Dutcher and Worts (1963), Leighton and Phillips (2003), and Siade and others (2014) for the Antelope Valley groundwater basin, and to Izbicki and others (1995) and Stamos and others (2001) for the Mojave Basin Area.
Faults partially control groundwater movement in parts of both groundwater basins (Stamos and others, 2001; Siade and others, 2014). Faults can be barriers or partial barriers to groundwater flow that could impede the flow of water across them, causing stairstep-like drops in the water table across the fault. Faults have been documented to function as a barriers to groundwater flow in water-bearing alluvial deposits in many desert basins (Londquist and Martin, 1991; Faunt, 1997) and are caused by the low permeability of the fault zone resulting from the compaction and deformation of the water-bearing deposits adjacent to the faults, and by lateral juxtaposition of high- and low-permeability units (Londquist and Martin, 1991). Cementation of the fault zone by the deposition of minerals from groundwater also can contribute to the reduction of fault-zone permeability. To the west of the study area, groundwater-level differences of more than $330 \mathrm{ft}$ over a distance of about $1.5 \mathrm{mi}$ on opposite sides of an unnamed fault were observed near Palmdale, Calif., in 2004 (Christensen, 2005).

In the study area, three faults - the Dibblee Unnamed Fault, another unnamed fault trending northwest from Three Sisters Buttes, referred to herein as the Dibblee Buttes Fault (Dibblee, 1959, 1960b; Bryant, 2005), and an unnamed fault between them from Siade and others (2014) _ extend across the alluvial deposits, but their southeastern extent and connection to the San Andreas Fault are unknown. Because of the unconsolidated character of the alluvium, the faults are not always clearly expressed on the land surface, and the extent of the three faults is only documented in the northwestern part of the study area. Owing to the paucity of groundwater-level data and lack of data on the geologic structure at depth, the effects of these faults on groundwater flow, if any, are unknown.

\section{Recharge}

The primary source of recharge to the study area is the infiltration of runoff from the San Gabriel Mountains to the south through many small ephemeral washes and stream channels. Runoff in the stream channel infiltrates the streambed and becomes groundwater recharge. The amount of runoff, or recharge, from the minor washes in the study area likely is very small, and there are no estimates of flow for most of these small stream channels. Siade and others (2014) estimated recharge to the groundwater system from Big Rock Wash, Mescal Creek and Jesus Canyon (combined), and Puzzle Canyon near the eastern boundary of the Antelope Valley groundwater basin (fig. 2) to be about 1,140, 440, and 400 acre-feet per year (acre-ft/yr), respectively. Stamos and others (2001) estimated recharge from Sheep Creek Wash to the El Mirage Valley groundwater basin to be about 1,940 acre-ft/yr.

Other possible sources of recharge to the study area are from direct infiltration of precipitation and lateral groundwater underflow from basement rocks and adjacent basins, which probably are small compared with the runoff from the San Gabriel Mountains (Leighton and Phillips, 2003). Because of the small amount of average annual precipitation in the area 
(about 5.05 inches per year [in/yr] in Palmdale, Calif., during 1934-2015; Western Regional Climate Center, 2015a) and high reference evapotranspiration rates (66.5 in/yr; California Irrigation Management Information System, 2015), recharge from direct infiltration of precipitation is considered negligible. Lateral groundwater flow from the basement complex and from other areas surrounding and adjacent to the study area also could be minor sources of recharge; however, Bloyd (1967), Leighton and Phillips (2003), and Siade and others (2014) estimated that the quantity of recharge from these sources, although uncertain, is also probably negligible.

\section{Discharge}

Evapotranspiration of water from the water table by phreatophytes is unlikely owing to the lack of springs or areas of shallow groundwater in the study area. Any evapotranspiration that occurs is by the transpiration of available pore water from the shallow root zone. El Mirage Lake (dry), in the northeastern part of the study area (fig. 2), is a topographic depression where water briefly ponds following intense precipitation or large flows from Sheep Creek Wash. Another possible natural source of discharge is lateral groundwater flow to adjacent areas. Previous studies in the Antelope Valley groundwater basin estimated that underflow to the El Mirage Valley groundwater basin was 100-500 acre-ft/yr (Bloyd, 1967), 1,000 acre-ft/yr (Durbin, 1978), and 400 acre-ft/yr (Leighton and Phillips, 2003); the most recent groundwaterflow model by Siade and others (2014) estimated that about $1,900 \mathrm{acre}-\mathrm{ft} / \mathrm{yr}$ of water flows eastward across the Antelope Valley groundwater basin boundary. Based on more recent groundwater-level data (discussed in the "Results of Field Reconnaissance Survey" section), the assumptions for the boundary conditions in the groundwater flow models - a general-head boundary in Siade and others (2014) and a no-flow boundary in Stamos and others (2001) - may not accurately represent the conditions of the groundwater system.

Historically, land use in the study area has been mostly undeveloped land with individual homesteads; therefore, withdrawal of groundwater by pumping for municipal water supply likely has been minimal. Irrigation districts were organized in the late 1890s in the western part of the study area to capture surface water near the mountain front, but these endeavors eventually failed because of the lack of a reliable water source (Thompson, 1929). Though hand-dug and flowing domestic wells were described by Thompson (1929) west of Big Rock Wash (fig. 2), domestic pumping in the study area has been minimal. There are no estimates of pumpage included in previous studies in the southeastern part of the Antelope Valley groundwater basin through 2005. Estimates of pumpage for the El Mirage Valley groundwater basin were less than 15 acre-ft/yr in the mid-1990s (Stamos and others, 2001).

Presently, some groundwater is extracted for domestic use within the study area and for irrigation in a few agricultural areas along the northern boundary of the study area, but most groundwater is extracted for municipal use from deep production wells along and near the Los Angeles-San Bernardino
County line. For the period of October 2013 to September 2014, the municipal pumpage within the study area was reported to be about 800 acre- $\mathrm{ft}$ in the Antelope Valley groundwater basin and about 2,080 acre-ft in the El Mirage Valley groundwater basin (Mojave Basin Area Watermaster, 2015).

\section{Gravity Surveys}

Gravity surveys are often completed in alluvial basins to define their thickness and to estimate the three-dimensional (3-D) geometry of the alluvial basin (Jachens and Moring, 1990; Saltus and Jachens, 1995). The thickness of the basinfill deposits (or depth to the basement complex) in the study area was estimated using the methods of Jachens and Moring (1990), modified to permit the inclusion of constraints at points where the thickness was known from direct observations in drill holes. Aeromagnetic data (Roberts and Jachens, 1999) were examined to discern the characteristics of the basement rocks at the base of the basin-fill deposits but were not directly used in this analysis presented in this report. For much of the western United States, including the Mojave Desert, Saltus and Jachens (1995) found a large density contrast, averaging -650 kilograms per cubic meter $\left(\mathrm{kg} / \mathrm{m}^{3}\right.$, -40.57 pounds per cubic foot $\left.\left[\mathrm{lb} / \mathrm{ft}^{3}\right]\right)$ in the upper $660 \mathrm{ft}$ of basin fill, between the basin-fill deposits and the crystalline basement complex, indicating that gravity methods would be appropriate for determining the thickness of the basin-fill deposits. A large density contrast $\left(-590 \mathrm{~kg} / \mathrm{m}^{3},-36.83 \mathrm{lb} / \mathrm{ft}^{3}\right)$ was corroborated for the Mojave Desert with deep drill holes in the western part of the Antelope Valley groundwater basin (Jachens and others, 2014). Geologic maps were used to define the contact of the basement complex and alluvial deposits at land surface, and data from drillers' logs that encountered or penetrated the basement complex were used to constrain the gravity-modeled thickness of the alluvial deposits.

\section{Previous Regional Basin Gravity Survey}

A regional basin gravity study was completed for the western Mojave Desert (Jachens and others, 2014) that included the Antelope Valley groundwater basin and covered a total area of about 7,500 $\mathrm{mi}^{2}$ with a grid of coarsely spaced data points. The basin gravity field reflects the threedimensional distribution of the basin fill for an area of about $2,100 \mathrm{mi}^{2}$, which is larger than the area in the present study shown in figure 3.

Preliminary analysis of the basin gravity field of the regional study area suggested a subsurface northeast-trending basement ridge and saddle (shaded oval in fig. 3) approximately $3.5 \mathrm{mi}$ west of the boundary between the Antelope Valley and El Mirage Valley groundwater basins along California State Route 18 (SR-18). Areas of potentially thinner alluvium are indicated by less negative gravity values. Because the data used in the regional gravity study by Jachens and others (2014) were widely spaced in most of the study 


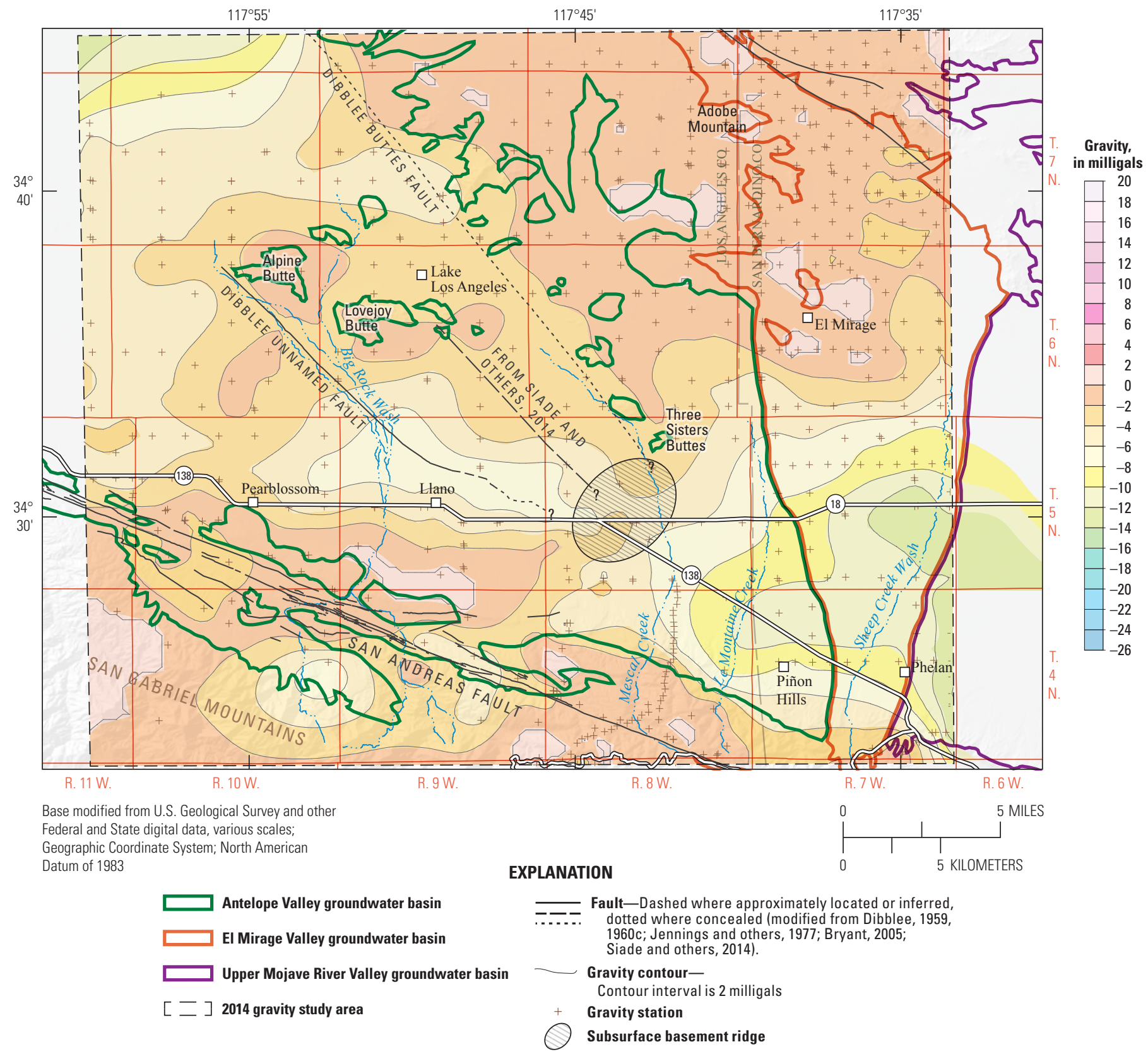

Figure 3. Regional basin gravity field near Piñon Hills, California (modified from figure 1-3 of Jachens and others, 2014). 
area (the spacing between measurements was greater than a mile), the estimated depth to basement using this gravity field data did not provide enough detail to support estimates of alluvial thickness in the area, locations of structures such as faults, or basement topography that can influence groundwater flow between the basins. However, the gravity field patterns in the regional survey suggested that structural features such as a subsurface ridge could be present, warranting the more detailed investigation by this study.

\section{Gravity Survey}

To augment the gravity data used in the regional gravity study by Jachens and others (2014) and provide better definition in the study area, the USGS completed a more detailed gravity survey by using more closely spaced measurements in 2014. Measurement spacing was as close as $220 \mathrm{ft}$ in some places to identify potential basement structures that could influence groundwater flow. The gravity measurements covered an area of about $200 \mathrm{mi}^{2}$, mostly between, and several miles north of the communities of Piñon Hills and Pearblossom, Calif. (fig. 4). For the hydrogeologic assessment, the 2014 gravity data were combined with the regional gravity data collected as part of other studies (Roberts and others, 1990; Jachens and others, 2014; figs. 3, 4).

\section{Gravity Survey Methods and Data}

Gravity measurements were collected at 413 new locations for this study (fig. 4; table 1) using a LaCoste and Romberg D-meter (D-79) relative gravity meter with Aliod 200x electronic feedback nulling system, with data resolution of 0.001 milligal ( $\mathrm{mGal}$ ) and repeatability of 0.01 to $0.02 \mathrm{mGal}$ (LaCoste and Romberg, 2003). The location and elevation of each gravity measurement were determined by using a Trimble Real-Time Kinematic (RTK) Model R7 and R8 GNSS Global Positioning System (GPS) base and rover receivers. This system is capable of obtaining vertical and horizontal coordinates with a precision of plus-or-minus $0.083 \mathrm{ft}$ between rover and base by using traditional RTK methods described by Rydlund and Densmore (2012). The RTK survey was referenced to the NAVD 88 by using surveyed locations and post-processing based on methods described by Rydlund and Densmore (2012) and processed using National Geodetic Survey Online Positioning User Service (OPUS) software (National Geodetic Survey, 2011).

The gravity data were tied to gravity benchmark CA475 (Roberts and Jachens, 1986), reduced using the Geodetic Reference System of 1967 (International Union of Geodesy and Geophysics, 1971), and referenced to the International Gravity Standardization Net 1971 gravity datum (Morelli, 1974; Hinze and others, 2005). The following description of the gravity analysis methods closely follows that of Martin (2011) and Flint and Martin (2012). Gravity data were adjusted using standard gravity corrections, including (1) the Earth tide correction, which corrects for tidal effects of the moon and sun; (2) instrument drift correction, which compensates for drift in the instrument's spring; (3) latitude correction, which corrects for the variation of the Earth's gravity with latitude; (4) free-air correction, which accounts for the variation in gravity with changes in elevation; (5) Bouguer correction, which corrects for the attraction of material between the station and the vertical datum; (6) curvature correction, which adjusts the Bouguer correction for the effect of the Earth's curvature; (7) terrain correction, which removes the effect of topography to a radial distance of about $104 \mathrm{mi}$; and (8) isostatic correction, which removes the long-wavelength effects of deep crustal and (or) upper mantle masses that isostatically support regional topography (Telford and others, 1990). Isostatic and terrain corrections beyond $104 \mathrm{mi}$ were interpolated from a grid generated from Karki and others (1961).

Terrain corrections were computed to a radial distance of $104 \mathrm{mi}$ and involved a three-part process: (1) Hayford-Bowie zones $\mathrm{A}$ and $\mathrm{B}$ with an outer radius of $223 \mathrm{ft}$ were estimated in the field with the aid of tables and charts from Robbins and Oliver (1970), (2) Hayford-Bowie zones C to M with an outer radius of $562 \mathrm{ft}$ were computed by using a digital elevation model, and (3) terrain corrections from a distance of $562 \mathrm{ft}$ to $104 \mathrm{mi}$ were calculated by using a digital elevation model and an established procedure by Plouff (1977). Total terrain corrections for the gravity stations used for this study, including the regional gravity measurements from Jachens and others (2014), ranged from 0.70 to $5.03 \mathrm{mGal}$, averaging $1.67 \mathrm{mGal}$; in comparison, isostatic residual gravity values (which account for low-density, deep-seated material below areas of high topography), ranged from about -41 to $-2 \mathrm{mGal}$.

\section{Geologic Maps}

Data from geologic maps were used primarily to delineate the contact between basin-fill deposits and outcrops of the basement complex. The 7.5- and 15-minute geologic quadrangle maps of the Mescal Creek (Dibblee, 2002a), Juniper Hills (Dibblee, 2002b), Shadow Mountains and Victorville (Dibblee, 2008), and Valyermo (Dibblee, 2002c) quadrangles were used for most of the study area, with additional information from the geologic maps of the Alpine Butte (Dibblee, 1959), Lancaster (Dibblee, 1960a), Shadow Mountains (Dibblee, 1960b), and Willow Springs and Rosamond quadrangles (Dibblee, 1963).

\section{Water-Well Logs}

Fifty-six drillers' logs and seismic geophysical data from the Los Angeles Regional Seismic Experiment (Fuis and others, 2001) containing information on the depth to the basement complex (fig. 4; table 2) were available to constrain the gravity model. These logs reported materials at the bottom of the drill hole consistent with the top of the basement complex, including consolidated and metamorphosed sandstone, shale, rock, or granite. 


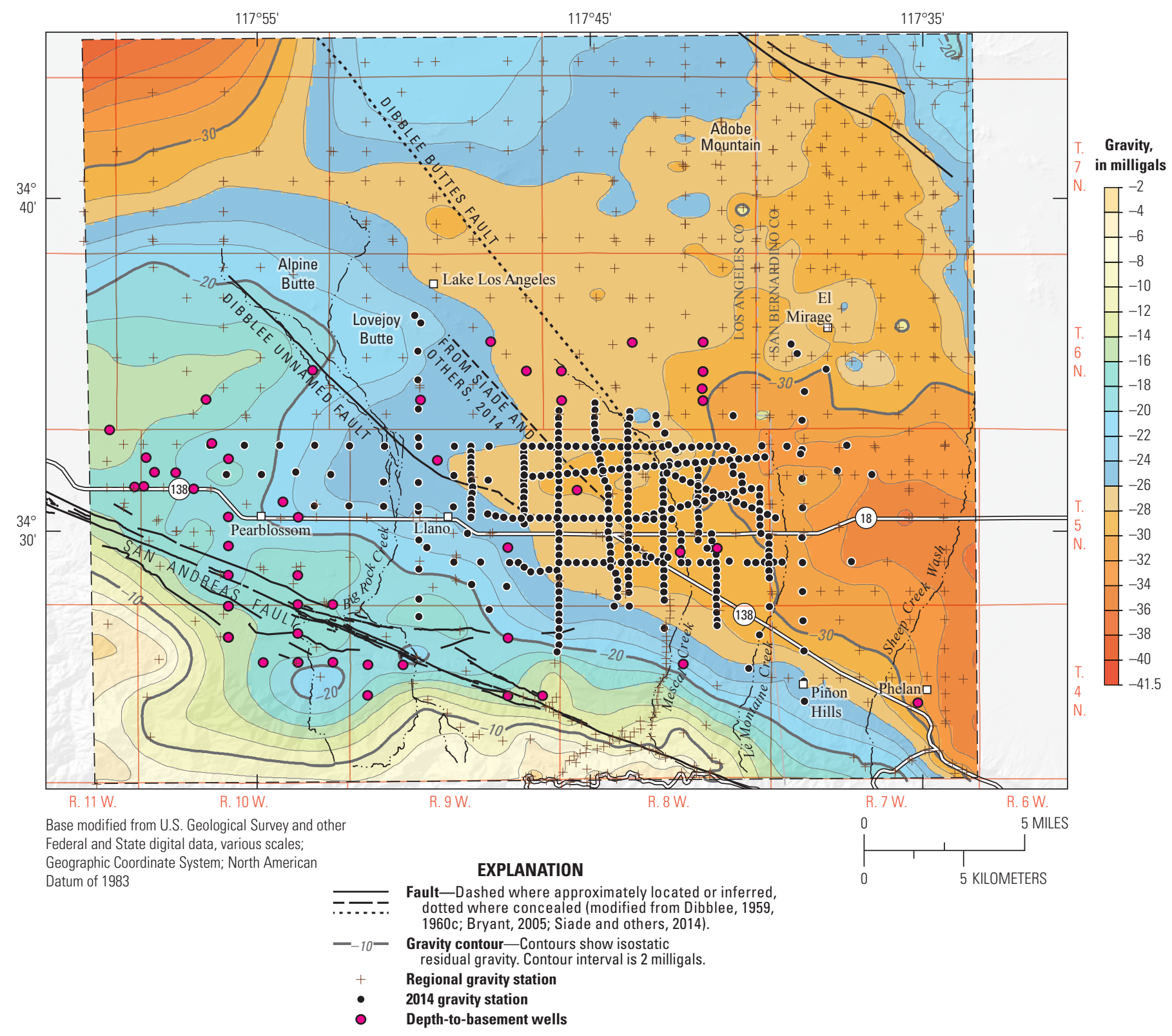

Figure 4. Location of gravity stations for the regional study (Jachens and others, 2014) and gravity stations and isostatic residual gravity field for this 2014 study, near Piñon Hills, California. 
Table 1. Gravity measurements collected in 2014 near Piñon Hills, California.

[See fig. 4 for well locations. Extent of inner-terrain correction 2,000 meters. Abbreviations: ft, foot; mGal, milligals; NAD 83, North American Datum of 1983; NAVD 88, North American Vertical Datum of 1988]

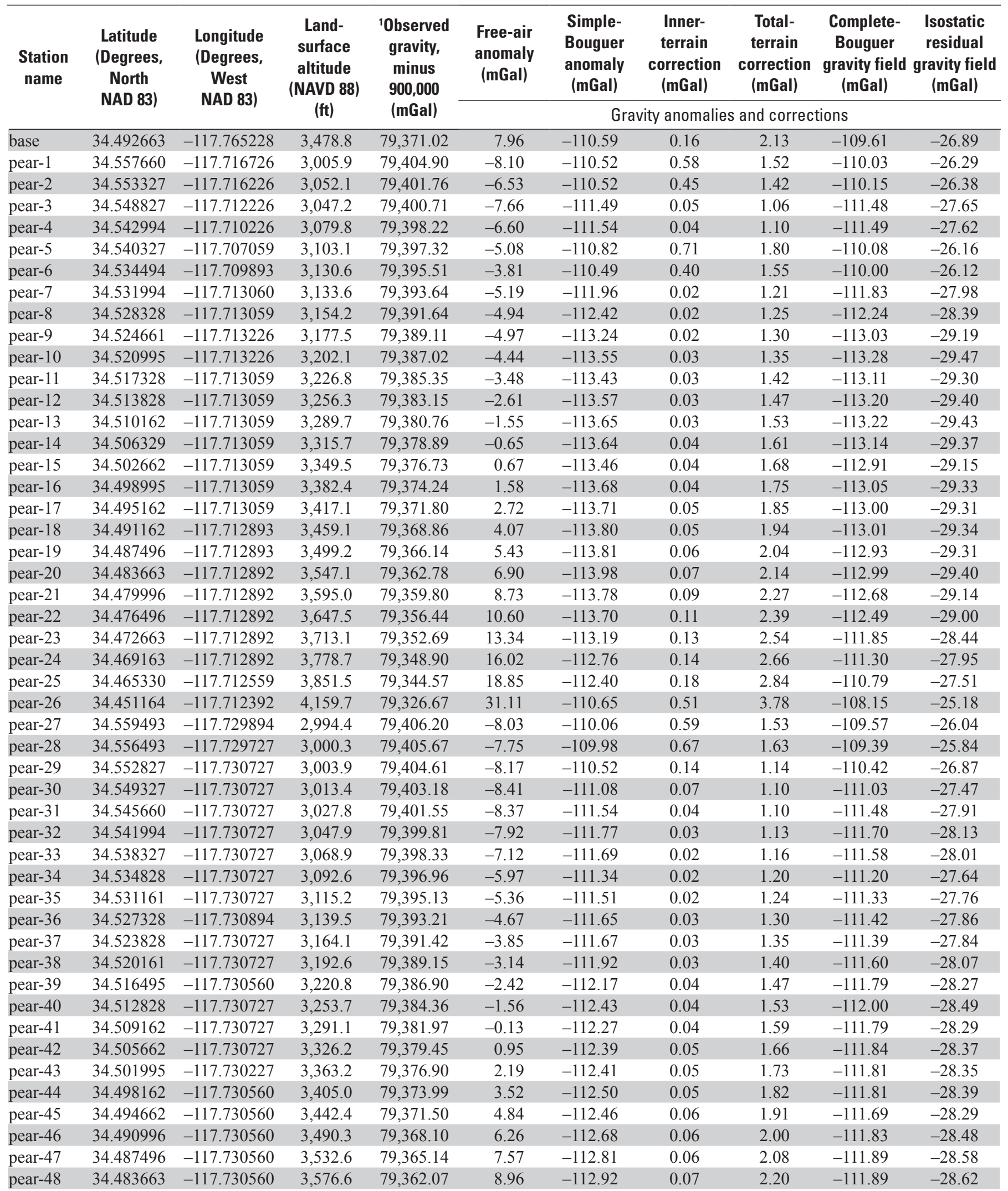


Table 1. Gravity measurements collected in 2014 near Piñon Hills, California.-Continued

[See fig. 4 for well locations. Extent of inner-terrain correction 2,000 meters. Abbreviations: ft, foot; mGal, milligals; NAD 83, North American Datum of 1983; NAVD 88, North American Vertical Datum of 1988]

\begin{tabular}{|c|c|c|c|c|c|c|c|c|c|c|}
\hline $\begin{array}{c}\text { Station } \\
\text { name }\end{array}$ & $\begin{array}{c}\text { Latitude } \\
\text { (Degrees, } \\
\text { North }\end{array}$ & $\begin{array}{l}\text { Longitude } \\
\text { (Degrees, } \\
\text { West }\end{array}$ & $\begin{array}{l}\text { Land- } \\
\text { surface } \\
\text { altitude } \\
\text { (NAVD 88) }\end{array}$ & $\begin{array}{c}\text { 'Observed } \\
\text { gravity, } \\
\text { minus } \\
900,000\end{array}$ & $\begin{array}{c}\text { Free-air } \\
\text { anomaly } \\
\text { (mGal) }\end{array}$ & $\begin{array}{c}\text { Simple- } \\
\text { Bouguer } \\
\text { anomaly } \\
\text { (mGal) }\end{array}$ & $\begin{array}{c}\text { Inner- } \\
\text { terrain } \\
\text { correction } \\
\text { (mGal) }\end{array}$ & $\begin{array}{c}\text { Total- } \\
\text { terrain } \\
\text { correction } \\
\text { (mGal) }\end{array}$ & $\begin{array}{l}\text { Complete- } \\
\text { Bouguer } \\
\text { gravity field } \\
\text { (mGal) }\end{array}$ & $\begin{array}{c}\text { Isostatic } \\
\text { residual } \\
\text { gravity field } \\
\text { (mGal) }\end{array}$ \\
\hline & & & (ft) & (mGal) & \multicolumn{6}{|c|}{ Gravity anomalies and corrections } \\
\hline pear-49 & 34.479996 & -117.730393 & $3,623.5$ & $79,358.75$ & 10.36 & -113.12 & 0.07 & 2.30 & -112.00 & -28.78 \\
\hline pear-50 & 34.476330 & -117.730393 & $3,672.1$ & $79,355.65$ & 12.14 & -113.00 & 0.09 & 2.44 & -111.75 & -28.58 \\
\hline pear-51 & 34.472830 & -117.730393 & $3,721.6$ & $79,352.80$ & 14.24 & -112.59 & 0.11 & 2.59 & -111.20 & -28.10 \\
\hline pear-52 & 34.468997 & -117.730226 & $3,775.7$ & $79,349.87$ & 16.72 & -111.95 & 0.14 & 2.75 & -110.42 & -27.39 \\
\hline pear-53 & 34.462164 & -117.730060 & $3,916.2$ & $79,341.99$ & 22.62 & -110.84 & 0.23 & 3.09 & -108.98 & -26.07 \\
\hline pear-54 & 34.484163 & -117.790062 & $3,490.3$ & $79,372.31$ & 11.04 & -107.89 & 0.13 & 2.33 & -106.72 & -24.57 \\
\hline pear-55 & 34.483996 & -117.785729 & $3,521.1$ & $79,370.00$ & 11.64 & -108.34 & 0.14 & 2.34 & -107.16 & -24.94 \\
\hline pear-56 & 34.481496 & -117.782562 & $3,580.2$ & $79,366.02$ & 13.43 & -108.57 & 0.15 & 2.42 & -107.32 & -25.08 \\
\hline pear-57 & 34.479496 & -117.779228 & $3,637.9$ & $79,362.09$ & 15.10 & -108.87 & 0.16 & 2.49 & -107.57 & -25.29 \\
\hline pear-58 & 34.479663 & -117.774562 & $3,663.2$ & $79,359.91$ & 15.28 & -109.55 & 0.14 & 2.46 & -108.28 & -25.93 \\
\hline pear-59 & 34.480330 & -117.770228 & $3,661.6$ & $79,359.57$ & 14.73 & -110.04 & 0.14 & 2.44 & -108.79 & \\
\hline pear-60 & 34.480330 & -117.766061 & $3,670.4$ & $79,358.54$ & 14.53 & -110.55 & 0.13 & 2.43 & -109.31 & -26.77 \\
\hline pear-61 & 34.484163 & -117.765061 & $3,605.5$ & $79,362.53$ & 12.10 & -110.77 & 0.12 & 2.31 & -109.64 & -27.03 \\
\hline pear-62 & 34.484163 & -117.760728 & $3,602.8$ & $79,362.31$ & 11.62 & -111.15 & 0.11 & 2.30 & -110.03 & -27.33 \\
\hline pear-63 & 34.483996 & -117.756394 & $3,597.6$ & 62.66 & 11.50 & -111.10 & 0.11 & 2.29 & -109.98 & 20 \\
\hline pear-64 & 34.483996 & -117.751894 & $3,589.1$ & $79,363.11$ & 11.15 & -11 & & 2.26 & -11 & -2 \\
\hline pear-65 & 34.483996 & -117.747394 & $3,578.6$ & $79,363.44$ & 10.49 & -111.46 & 0.09 & 2.25 & -110.38 & -27.42 \\
\hline pear-66 & 34.483996 & -117.742727 & $3,563.8$ & $79,363.80$ & 9.46 & -111.98 & 0.09 & 2.24 & -110.91 & -27.85 \\
\hline pear-67 & 34.483996 & -117.738560 & $3,566.7$ & 79 & 9.12 & -112.42 & 0.07 & 2.21 & -111.37 & -28.23 \\
\hline pear-68 & 34.483996 & -117.734060 & $3,574.0$ & 362.43 & 9.05 & -112.74 & 0.07 & 2.20 & -111.72 & \\
\hline pear-69 & 34.483996 & -117.729560 & $3,574.0$ & $79,362.06$ & 8.68 & -113.11 & 0.06 & 2.17 & -112.11 & -28.83 \\
\hline pear-70 & 34.483996 & -117.725226 & $3,564.5$ & $79,362.29$ & 8.01 & -113.45 & 0.07 & 2.17 & -112.45 & -29.08 \\
\hline pear-71 & 34.483996 & -117.721726 & $3,554.6$ & $79,362.76$ & 7.55 & -113.57 & 0.07 & 2.16 & -112.58 & -29.15 \\
\hline pear-72 & 34.487329 & -117.721726 & $3,516.2$ & 75 & 6.25 & -113.57 & 0.06 & 2.06 & -112.66 & -2 \\
\hline pear-73 & 34.486663 & -117.719059 & $3,518.8$ & $79,365.07$ & 6.2 & -113.63 & 0.06 & 2.07 & -112.72 & -29.21 \\
\hline pear-74 & 34.484663 & -117.715559 & $3,534.6$ & $79,363.81$ & 6.67 & -113.78 & 0.07 & 2.13 & -112.81 & -29.26 \\
\hline pear-75 & 34.483663 & -117.712892 & $3,547.4$ & $79,362.92$ & 7.07 & -113.82 & 0.07 & 2.14 & -112.84 & -29.25 \\
\hline pear-76 & 34.486663 & -117.710559 & $3,507.7$ & $79,365.52$ & 5.68 & -113.85 & 0.06 & 2.05 & -112.96 & -29.31 \\
\hline pear-77 & 34.483996 & -117.703892 & $3,535.3$ & 63.85 & 683 & -113.64 & 0.07 & 2.10 & -112.70 & -28.97 \\
\hline pear-78 & 34.483996 & -117.700392 & $3,540.8$ & 63.69 & 7. & -113.47 & 0 & 2.07 & -112.56 & -28.77 \\
\hline pear-79 & 34.483996 & -117.694225 & $3,547.4$ & $79,363.23$ & 7.35 & -113.54 & 0.06 & 2.05 & -112.65 & -28.75 \\
\hline pear- 80 & 34.483996 & -117.689725 & $3,546.4$ & $79,363.13$ & 7.15 & -113.70 & 0.06 & 2.02 & -112.84 & -28.87 \\
\hline pear-81 & 34.483996 & -117.686391 & $3,542.8$ & $79,363.23$ & 6.91 & -113.81 & 0.06 & 2.01 & -112.97 & -28.95 \\
\hline pear-82 & 34.484829 & -117.679891 & $3,530.7$ & $79,363.84$ & 6.32 & -114.00 & 0.05 & 1.95 & -113.21 & -29.10 \\
\hline pear-83 & 4.483996 & -117.670891 & $3,525.4$ & $79,363.94$ & 50 & -114.14 & 0.0 & 1.91 & -113.39 & -29.14 \\
\hline pear- 84 & 34.484162 & -117.666557 & $3,511.6$ & $79,364.38$ & 5.12 & -114.54 & 0.04 & 1.89 & -113.81 & -29.50 \\
\hline pear-85 & 34.484162 & -117.662223 & $3,500.5$ & $79,364.80$ & 4.49 & -114.79 & 0.04 & 1.87 & -114.08 & -29.70 \\
\hline pear-86 & 34.484162 & -117.657723 & $3,491.6$ & $79,364.96$ & 3.82 & -115.16 & 0.04 & 1.85 & -114.47 & -30.03 \\
\hline pear-87 & 34.484162 & -117.653556 & $3,486.7$ & $79,364.84$ & 3.24 & -115.58 & 0.04 & 1.83 & -114.90 & -30.40 \\
\hline pear- 88 & 4.484162 & -117.643389 & $3,493.3$ & $79,363.30$ & 2.32 & -116.72 & 0.03 & 1.76 & -116.11 & -31.46 \\
\hline pear-89 & 34.484662 & -117.618555 & $3,552.0$ & $79,356.60$ & 1.0 & -119.95 & 0.03 & 1.64 & -119.47 & -34.54 \\
\hline pear-90 & 34.542327 & -117.809230 & $2,926.8$ & $79,410.82$ & -8.32 & -108.04 & 0.02 & 1.16 & -107.90 & -25.74 \\
\hline pear-91 & 34.539494 & -117.809230 & $2,941.5$ & $79,409.34$ & -8.18 & -108.40 & 0.02 & 1.20 & -108.23 & -26.07 \\
\hline pear-92 & 34.536660 & -117.809230 & $2,958.3$ & $79,407.78$ & -7.92 & -108.72 & 0.02 & 1.23 & -108.51 & -26.35 \\
\hline pear-93 & 34.533661 & -117.809230 & $2,977.6$ & $79,406.03$ & -7.60 & -109.06 & 0.02 & 1.27 & -108.82 & -26.66 \\
\hline pear-94 & 34.530827 & -117.809230 & $2,997.7$ & $79,404.33$ & -7.17 & -109.31 & 0.03 & 1.31 & -109.04 & -26.89 \\
\hline pear-95 & 34.527994 & -117.809230 & $3,018.1$ & $79,402.75$ & -6.60 & -109.44 & 0.03 & 1.35 & -109.13 & -27.00 \\
\hline pear-96 & 34.525161 & -117.809063 & $3,037.4$ & $79,401.40$ & -5.90 & -109.39 & 0.04 & 1.40 & -109.04 & -26.92 \\
\hline pear-97 & 34.521828 & -117.808563 & $3,070.3$ & $79,399.55$ & -4.38 & -108.99 & 0.07 & 1.48 & -108.57 & -26.47 \\
\hline
\end{tabular}


Table 1. Gravity measurements collected in 2014 near Piñon Hills, California.-Continued

[See fig. 4 for well locations. Extent of inner-terrain correction 2,000 meters. Abbreviations: ft, foot; mGal, milligals; NAD 83, North American Datum of 1983; NAVD 88, North American Vertical Datum of 1988]

\begin{tabular}{|c|c|c|c|c|c|c|c|c|c|c|}
\hline $\begin{array}{c}\text { Station } \\
\text { name }\end{array}$ & $\begin{array}{l}\text { Latitude } \\
\text { (Degrees, } \\
\text { North }\end{array}$ & $\begin{array}{c}\text { Longitude } \\
\text { (Degrees, } \\
\text { West }\end{array}$ & $\begin{array}{l}\text { Land- } \\
\text { surface } \\
\text { altitude } \\
\text { (NAVD 88) }\end{array}$ & $\begin{array}{c}\text { 'Observed } \\
\text { gravity, } \\
\text { minus } \\
900,000\end{array}$ & $\begin{array}{c}\text { Free-air } \\
\text { anomaly } \\
\text { (mGal) }\end{array}$ & $\begin{array}{c}\text { Simple- } \\
\text { Bouguer } \\
\text { anomaly } \\
\text { (mGal) }\end{array}$ & $\begin{array}{l}\text { Inner- } \\
\text { terrain } \\
\text { correction } \\
\text { (mGal) }\end{array}$ & $\begin{array}{l}\text { Total- } \\
\text { terrain } \\
\text { correction } \\
\text { (mGal) }\end{array}$ & $\begin{array}{l}\text { Complete- } \\
\text { Bouguer } \\
\text { gravity field } \\
\text { (mGal) }\end{array}$ & $\begin{array}{c}\text { Isostatic } \\
\text { residual } \\
\text { gravity field } \\
\text { (mGal) }\end{array}$ \\
\hline & & & (ft) & (mGal) & \multicolumn{6}{|c|}{ Gravity anomalies and corrections } \\
\hline pear-98 & 34.518494 & -117.808730 & $3,110.0$ & $79,397.73$ & -2.18 & -108.15 & 0.07 & 1.53 & -107.69 & -25.61 \\
\hline pear-99 & 34.515661 & -117.809230 & $3,146.7$ & $79,395.66$ & -0.56 & -107.78 & 0.03 & 1.53 & -107.33 & -25.28 \\
\hline pear-102 & 34.506828 & -117.809230 & $3,198.9$ & $79,392.29$ & 1.72 & -107.28 & 0.04 & 1.69 & -106.67 & -24.67 \\
\hline pear-103 & 34.542160 & -117.782896 & $2,958.3$ & $79,408.00$ & -8.16 & -108.96 & 0.02 & 1.16 & -108.83 & -26.14 \\
\hline pear-104 & 34.539161 & -117.782896 & $2,975.3$ & $79,406.33$ & -7.98 & -109.36 & 0.02 & 1.20 & -109.19 & -26.51 \\
\hline pear-105 & 34.536327 & -117.782896 & $2,992.1$ & $79,404.74$ & -7.75 & -109.70 & 0.03 & 1.24 & -109.50 & -26.83 \\
\hline pear-106 & 34.533494 & -117.782896 & $3,011.5$ & $79,402.81$ & -7.63 & -110.24 & 0.03 & 1.28 & -110.00 & -27.33 \\
\hline pear-111 & 34.519328 & -117.782895 & $3,134.6$ & $79,393.96$ & -3.71 & -110.52 & 0.05 & 1.49 & -110.09 & -27.50 \\
\hline pear-112 & 34.516661 & -117.782895 & $3,161.1$ & $79,391.99$ & -2.96 & -110.67 & 0.05 & 1.54 & -110.21 & -27.64 \\
\hline pear-113 & 34.513162 & -117.782895 & $3,193.9$ & $79,389.91$ & -1.66 & -110.49 & 0.06 & 1.62 & -109.96 & -27.40 \\
\hline pear-114 & 34.510162 & -117.782729 & $3,230.0$ & $79,387.94$ & 0.02 & -110.05 & 0.07 & 1.67 & -109.46 & -26.90 \\
\hline pear-115 & 34.506995 & -117.782729 & $3,285.5$ & $79,384.85$ & 2.41 & -109.54 & 0.09 & 1.75 & -108.90 & -26.37 \\
\hline pear-116 & 34.503495 & -117.782729 & $3,317.3$ & $79,383.06$ & 3.91 & -109.13 & 0.10 & 1.83 & -108.42 & -25.91 \\
\hline pear-117 & 34.498329 & -117.688725 & $3,394.7$ & $79,373.31$ & 1.87 & -113.81 & 0.04 & 1.69 & -113.25 & -29.15 \\
\hline pear-118 & 34.494829 & -117.688725 & $3,429.0$ & $79,371.09$ & 3.16 & -113.68 & 0.04 & 1.76 & -113.06 & -28.98 \\
\hline pear-119 & 34.490996 & -117.688725 & $3,469.0$ & $79,368.32$ & 4.48 & -113.73 & 0.05 & 1.85 & -113.03 & -28.98 \\
\hline pear-120 & 34.487996 & -117.686391 & $3,499.5$ & $79,366.27$ & 5.55 & -113.70 & 0.05 & 1.90 & -112.95 & -28.89 \\
\hline pear-121 & 34.483996 & -117.686558 & $3,543.1$ & $79,363.14$ & 6.85 & -113.88 & 0.06 & 2.01 & -113.04 & -29.02 \\
\hline pear-129 & 34.455164 & -117.686058 & $3,902.4$ & $79,339.93$ & 19.86 & -113.13 & 0.17 & 3.05 & -111.32 & -27.74 \\
\hline pear-130 & 34.452330 & -117.686058 & $3,968.0$ & $79,335.76$ & 22.09 & -113.13 & 0.20 & 3.21 & -111.18 & -27.67 \\
\hline pear-131 & 34.498329 & -117.704059 & $3,387.9$ & $79,373.78$ & 1.69 & -113.75 & 0.04 & 1.75 & -113.13 & -29.27 \\
\hline pear-132 & 34.494829 & -117.704059 & $3,421.1$ & $79,371.56$ & 2.89 & -113.69 & 0.04 & 1.82 & -113.00 & -29.16 \\
\hline pear-133 & 34.491162 & -117.704059 & $3,454.5$ & $79,369.08$ & 3.86 & -113.86 & 0.05 & 1.91 & -113.09 & -29.28 \\
\hline pear-134 & 34.487496 & -117.704059 & $3,495.2$ & $79,366.40$ & 5.31 & -113.79 & 0.06 & 2.00 & -112.94 & -29.17 \\
\hline pear-135 & 34.483996 & -117.703892 & $3,535.3$ & $79,363.78$ & 6.76 & -113.71 & 0.07 & 2.10 & -112.77 & -29.04 \\
\hline pear-136 & 34.560160 & -117.765228 & $2,912.3$ & $79,412.01$ & -10.00 & -109.23 & 0.01 & 0.96 & -109.29 & -26.33 \\
\hline pear-137 & 34.556493 & -117.765395 & $2,924.8$ & $79,410.49$ & -10.03 & -109.69 & 0.01 & 0.99 & -109.72 & -26.75 \\
\hline pear-138 & 34.552827 & -117.765395 & $2,942.2$ & $79,408.74$ & -9.84 & -110.09 & 0.02 & 1.03 & -110.08 & -27.09 \\
\hline pear-139 & 34.549160 & -117.765395 & $2,960.2$ & $79,407.18$ & -9.40 & -110.26 & 0.02 & 1.10 & -110.18 & -27.20 \\
\hline pear-140 & 34.545827 & -117.765395 & $2,977.3$ & $79,405.74$ & -8.95 & -110.39 & 0.02 & 1.10 & -110.32 & -27.34 \\
\hline pear-141 & 34.542494 & -117.765395 & $2,996.0$ & $79,404.31$ & -8.34 & -110.42 & 0.02 & 1.14 & -110.32 & -27.34 \\
\hline pear-142 & 34.538827 & -117.765395 & $3,019.7$ & $79,402.41$ & -7.71 & -110.60 & 0.02 & 1.18 & -110.46 & -27.48 \\
\hline pear-143 & 34.535328 & -117.765228 & $3,038.4$ & $79,400.73$ & -7.33 & -110.86 & 0.02 & 1.22 & -110.68 & -27.70 \\
\hline pear-144 & 34.531661 & -117.765228 & $3,062.7$ & $79,398.54$ & -6.93 & -111.28 & 0.03 & 1.29 & -111.05 & -28.07 \\
\hline pear-145 & 34.528328 & -117.765228 & $3,088.0$ & $79,396.54$ & -6.27 & -111.49 & 0.03 & 1.34 & -111.21 & -28.22 \\
\hline pear-146 & 34.524661 & -117.765228 & $3,115.9$ & $79,394.53$ & -5.35 & -111.52 & 0.04 & 1.40 & -111.18 & -28.21 \\
\hline
\end{tabular}


Table 1. Gravity measurements collected in 2014 near Piñon Hills, California.-Continued

[See fig. 4 for well locations. Extent of inner-terrain correction 2,000 meters. Abbreviations: ft, foot; mGal, milligals; NAD 83, North American Datum of 1983; NAVD 88, North American Vertical Datum of 1988]

\begin{tabular}{|c|c|c|c|c|c|c|c|c|c|c|}
\hline $\begin{array}{c}\text { Station } \\
\text { name }\end{array}$ & $\begin{array}{c}\text { Latitude } \\
\text { (Degrees, } \\
\text { North }\end{array}$ & $\begin{array}{l}\text { Longitude } \\
\text { (Degrees, } \\
\text { West }\end{array}$ & $\begin{array}{l}\text { Land- } \\
\text { surface } \\
\text { altitude } \\
\text { (NAVD 88) }\end{array}$ & $\begin{array}{c}\text { 'Observed } \\
\text { gravity, } \\
\text { minus } \\
900,000\end{array}$ & $\begin{array}{c}\text { Free-air } \\
\text { anomaly } \\
\text { (mGal) }\end{array}$ & $\begin{array}{c}\text { Simple- } \\
\text { Bouguer } \\
\text { anomaly } \\
\text { (mGal) }\end{array}$ & $\begin{array}{c}\text { Inner- } \\
\text { terrain } \\
\text { correction } \\
\text { (mGal) }\end{array}$ & $\begin{array}{c}\text { Total- } \\
\text { terrain } \\
\text { correction } \\
\text { (mGal) }\end{array}$ & $\begin{array}{l}\text { Complete- } \\
\text { Bouguer } \\
\text { gravity field } \\
\text { (mGal) }\end{array}$ & $\begin{array}{c}\text { Isostatic } \\
\text { residual } \\
\text { gravity field } \\
\text { (mGal) }\end{array}$ \\
\hline & & & (ft) & (mGal) & \multicolumn{6}{|c|}{ Gravity anomalies and corrections } \\
\hline pear-147 & 34.520828 & -117.765228 & $3,148.0$ & $79,392.32$ & -4.21 & -111.48 & 0.04 & 1.45 & -111.10 & -28.16 \\
\hline pear-148 & 34.517162 & -117.765395 & $3,184.8$ & $79,390.00$ & -2.77 & -111.28 & 0.04 & 1.51 & -110.86 & -27.95 \\
\hline pear-149 & 34.513328 & -117.765228 & $3,221.2$ & $79,387.76$ & -1.26 & -111.02 & 0.05 & 1.58 & -110.53 & -27.62 \\
\hline pear-150 & 34.509662 & -117.765228 & $3,256.0$ & $79,385.71$ & 0.27 & -110.68 & 0.06 & 1.66 & -110.11 & -27.22 \\
\hline pear-151 & 34.506162 & -117.765228 & $3,297.6$ & $79,383.07$ & 1.84 & -110.53 & 0.07 & 1.74 & -109.90 & -27.05 \\
\hline pear- 152 & 34.502496 & -117.765228 & $3,344.5$ & $79,380.24$ & 3.73 & -110.24 & 0.08 & 1.82 & -109.54 & -26.70 \\
\hline pear-153 & 34.498829 & -117.765228 & $3,399.0$ & $79,376.65$ & 5.57 & -110.25 & 0.08 & 1.90 & -109.49 & -26.70 \\
\hline pear-154 & 34.495329 & -117.765228 & $3,442.1$ & $79,373.64$ & 6.90 & -110.39 & 0.08 & 1.99 & -109.55 & -26.80 \\
\hline pear-155 & 34.491329 & -117.765228 & $3,492.6$ & $79,370.11$ & 8.46 & -110.56 & 0.10 & 2.11 & -109.60 & -26.89 \\
\hline pear-156 & 34.487663 & -117.765228 & $3,547.4$ & $79,366.37$ & 10.18 & -110.70 & 0.11 & 2.20 & -109.66 & -27.01 \\
\hline pear-157 & 34.484163 & -117.765228 & $3,605.8$ & $79,362.56$ & 12.15 & -110.72 & 0.12 & 2.31 & -109.59 & -2 \\
\hline pear-158 & 34.480330 & -117.766061 & $3,670.1$ & $79,358.54$ & 14.50 & -110.56 & 0.13 & 2.43 & -109.32 & -26.78 \\
\hline pear-159 & 34.476496 & -117.765228 & $3,738.7$ & $79,354.39$ & 17.13 & -110.28 & 0.14 & 2.56 & -108.93 & -26.45 \\
\hline pear- 160 & 34.472830 & -117.765061 & $3,802.3$ & 350.67 & 19.70 & -109.88 & 0.15 & 2.68 & -108.42 & -26.01 \\
\hline pear-161 & 34.469163 & -117.765061 & $3,872.5$ & 46.43 & 22.36 & -109.61 & & & -108.01 & \\
\hline pear-162 & 34.465663 & -117.765061 & $3,939.5$ & $79,342.61$ & 25.14 & -10 & & 2.97 & -10 & \\
\hline pear-163 & 34.461997 & -117.765061 & $4,005.1$ & $79,338.66$ & 27.67 & -108.83 & 0.19 & 3.13 & -106.96 & -24.77 \\
\hline pear-164 & 34.458164 & -117.765061 & $4,079.0$ & $79,334.08$ & 30.35 & -108.66 & 0.21 & 3.31 & -106.62 & -24.53 \\
\hline pear-165 & 34.454831 & -117.765061 & $4,142.3$ & 0.34 & 32 & 33 & 0.22 & 3.47 & -106.14 & 12 \\
\hline pear-166 & 34.450997 & -117.765061 & $4,213.2$ & 26.71 & 36.20 & -107.39 & 0.23 & 3.64 & -105.04 & \\
\hline pear-167 & 34.446997 & -117.765061 & $4,290.3$ & $79,322.51$ & 39.58 & -106.63 & 0.31 & 3.91 & -104.03 & -22.22 \\
\hline pear-168 & 34.442998 & -117.765061 & $4,378.9$ & $79,318.31$ & 44.05 & -105.19 & 0.50 & 4.26 & -102.25 & -20.56 \\
\hline pear-169 & 34.439331 & -117.766227 & $4,445.5$ & $79,315.69$ & 48.00 & -103.51 & 0.87 & 4.79 & -100.05 & -18.49 \\
\hline pear-170 & 34.563993 & -117.747228 & $2,926.8$ & 79 & -11.07 & -11 & 0 & 0.93 & -11 & 65 \\
\hline pear-171 & 34.560327 & -117.747061 & $2,940.2$ & $79,408.67$ & -10.73 & -110.91 & 0 & 0.97 & -110.97 & -27.73 \\
\hline pear-172 & 34.556660 & -117.747228 & $2,956.6$ & $79,407.29$ & -10.26 & -111.00 & 0.02 & 1.00 & -111.03 & -27.78 \\
\hline pear-173 & 34.553160 & -117.747228 & $2,970.7$ & $79,406.01$ & -9.92 & -111.14 & 0.02 & 1.03 & -111.14 & -27.88 \\
\hline pear-174 & 34.549660 & -117.747061 & $2,989.8$ & $79,404.62$ & -9.21 & -111.09 & 0.01 & 1.05 & -111.07 & -27.78 \\
\hline pear-175 & 34.546161 & -117.746561 & $3,006.5$ & $79,403.08$ & -8.89 & -111.33 & 002 & 1.10 & -111.27 & -27.97 \\
\hline pear-176 & 34.542494 & -117.745894 & $3,027.9$ & 01.44 & -8.22 & -111.38 & 0 & 1. & -111.30 & -27.98 \\
\hline pear-177 & 34.538994 & -117.745561 & $3,047.0$ & $79,399.88$ & -7.69 & -111.50 & 0.02 & 1.17 & -111.39 & -28.05 \\
\hline pear-178 & 34.535494 & -117.744728 & $3,069.9$ & $79,398.00$ & -7.12 & -111.72 & 0.02 & 1.21 & -111.57 & -28.22 \\
\hline pear-179 & 34.531994 & -117.744228 & $3,094.2$ & $79,396.14$ & -6.40 & -111.83 & 0.02 & 1.25 & -111.64 & -28.29 \\
\hline pear-180 & 34.528495 & -117.743394 & $3,119.5$ & $79,394.48$ & -5.38 & -111.67 & 0.03 & 1.31 & -111.43 & -28.08 \\
\hline pear-181 & 34.524995 & -117.742394 & $3,147.7$ & $79,392.56$ & -4.35 & -111.61 & 0 & 1. & -111.33 & -27.99 \\
\hline pear-182 & 34.521495 & -117.740561 & $3,175.6$ & $79,390.33$ & -3.67 & -111.87 & 0.03 & 1.40 & -111.55 & -28.20 \\
\hline pear-183 & 34.517828 & -117.740227 & $3,208.4$ & $79,387.94$ & -2.66 & -111.99 & 0.03 & 1.45 & -111.62 & -28.27 \\
\hline pear-184 & 34.513995 & -117.740227 & $3,239.9$ & $79,385.85$ & -1.47 & -111.87 & 0.04 & 1.53 & -111.44 & -28.10 \\
\hline pear-185 & 34.510329 & -117.740561 & $3,275.3$ & $79,383.58$ & -0.10 & -111.70 & 0.04 & 1.59 & -111.22 & -27.90 \\
\hline pear-186 & 34.506662 & -117.739894 & $3,308.1$ & $79,381.42$ & 1.13 & -111.59 & 0.05 & 1.66 & -111.04 & -27.72 \\
\hline pear-187 & 34.503162 & -117.740061 & $3,344.2$ & $79,378.92$ & 2. & -111.63 & 0.0 & 1.74 & -111.01 & -27.71 \\
\hline pear-188 & 34.499496 & -117.740561 & $3,385.3$ & $79,376.31$ & 3.88 & -111.48 & 0.05 & 1.82 & -110.79 & -27.53 \\
\hline pear-189 & 34.495829 & -117.740227 & $3,420.8$ & $79,373.60$ & 4.82 & -111.75 & 0.06 & 1.90 & -110.98 & -27.75 \\
\hline pear-190 & 34.492496 & -117.739394 & $3,460.4$ & $79,370.72$ & 5.94 & -111.98 & 0.07 & 1.99 & -111.13 & -27.91 \\
\hline pear-191 & 34.488829 & -117.739394 & $3,504.1$ & $79,367.76$ & 7.40 & -112.01 & 0.07 & 2.08 & -111.08 & -27.90 \\
\hline pear-192 & 34.485330 & -117.739394 & & $79,364.67$ & 873 & -112.17 & 0.07 & 2.18 & -111.16 & -28.02 \\
\hline pear-193 & 34.481663 & -117.739227 & $3,588.7$ & $79,361.88$ & 10.08 & -112.21 & 0.09 & 2.30 & -111.09 & -28.00 \\
\hline pear-194 & 34.478663 & -117.737060 & $3,635.3$ & $79,358.64$ & 11.47 & -112.41 & 0.09 & 2.39 & -111.21 & -28.13 \\
\hline pear-195 & 34.474830 & -117.735893 & $3,689.5$ & $79,355.25$ & 13.50 & -112.23 & 0.11 & 2.53 & -110.90 & -27.86 \\
\hline
\end{tabular}


Table 1. Gravity measurements collected in 2014 near Piñon Hills, California.-Continued

[See fig. 4 for well locations. Extent of inner-terrain correction 2,000 meters. Abbreviations: ft, foot; mGal, milligals; NAD 83, North American Datum of 1983; NAVD 88, North American Vertical Datum of 1988]

\begin{tabular}{|c|c|c|c|c|c|c|c|c|c|c|}
\hline $\begin{array}{l}\text { Station } \\
\text { name }\end{array}$ & $\begin{array}{l}\text { Latitude } \\
\text { (Degrees, } \\
\text { North }\end{array}$ & $\begin{array}{l}\text { Longitude } \\
\text { (Degrees, } \\
\text { West }\end{array}$ & $\begin{array}{l}\text { Land- } \\
\text { surface } \\
\text { altitude } \\
\text { (NAVD 88) }\end{array}$ & $\begin{array}{c}\text { 'Observed } \\
\text { gravity, } \\
\text { minus } \\
900,000\end{array}$ & $\begin{array}{l}\text { Free-air } \\
\text { anomaly } \\
\text { (mGal) }\end{array}$ & $\begin{array}{c}\text { Simple- } \\
\text { Bouguer } \\
\text { anomaly } \\
\text { (mGal) }\end{array}$ & $\begin{array}{l}\text { Inner- } \\
\text { terrain } \\
\text { correction } \\
\text { (mGal) }\end{array}$ & $\begin{array}{l}\text { Total- } \\
\text { terrain } \\
\text { correction } \\
\text { (mGal) }\end{array}$ & $\begin{array}{l}\text { Complete- } \\
\text { Bouguer } \\
\text { gravity field } \\
\text { (mGal) }\end{array}$ & $\begin{array}{l}\text { Isostatic } \\
\text { residual } \\
\text { gravity field } \\
\text { (mGal) }\end{array}$ \\
\hline & & & (ft) & (mGal) & \multicolumn{6}{|c|}{ Gravity anomalies and corrections } \\
\hline pear-196 & 34.471163 & -117.735893 & $3,758.0$ & $79,351.20$ & 16.20 & -111.87 & 0.13 & 2.67 & -110.40 & -27.44 \\
\hline pear-197 & 34.466830 & -117.735893 & $3,841.4$ & $79,346.62$ & 19.83 & -111.08 & 0.17 & 2.87 & -109.44 & -26.56 \\
\hline pear-200 & 34.588658 & -117.646057 & $2,969.1$ & $79,408.43$ & -10.64 & -111.81 & 0.54 & 1.20 & -111.64 & -27.11 \\
\hline pear-201 & 34.580658 & -117.631390 & $2,983.5$ & $79,404.18$ & -12.86 & -114.52 & 0.01 & 0.70 & -114.85 & -30.09 \\
\hline pear-202 & 34.569659 & -117.642223 & $3,023.2$ & $79,399.98$ & -12.40 & -115.41 & 0.01 & 0.79 & -115.67 & -31.02 \\
\hline pear-203 & 34.555160 & -117.643556 & $3,087.2$ & $79,393.35$ & -11.79 & -116.98 & 0.01 & 0.88 & -117.16 & -32.45 \\
\hline pear-204 & 34.540660 & -117.643390 & $3,153.8$ & $79,387.20$ & -10.45 & -117.92 & 0.01 & 1.00 & -117.99 & -33.20 \\
\hline pear-209 & 34.469496 & -117.643223 & $3,623.5$ & $79,354.33$ & 6.83 & -116.65 & 0.04 & 2.09 & -115.74 & -31.29 \\
\hline pear-210 & 34.454997 & -117.642889 & $3,791.2$ & $79,343.29$ & 12.78 & -116.42 & 0.09 & 2.55 & -115.09 & -30.85 \\
\hline pear-211 & 34.439664 & -117.642556 & $4,043.2$ & $79,329.10$ & 23.56 & -114.23 & 0.17 & 3.21 & -112.28 & -28.35 \\
\hline pear-212 & 34.424498 & -117.642556 & $4,379.3$ & $79,309.43$ & 36.76 & -112.49 & 0.35 & 4.11 & -109.70 & -26.25 \\
\hline pear-213 & 34.414665 & -117.642389 & $4,668.7$ & $79,292.90$ & 48.26 & -110.86 & 0.68 & 5.03 & -107.18 & -24.08 \\
\hline pear-214 & 34.542826 & -117.940067 & $2,798.8$ & $79,428.19$ & -3.03 & -98.39 & 0.02 & 1.16 & -98.21 & -18.85 \\
\hline pear-215 & 34.542659 & -117.922733 & $2,808.7$ & $79,427.39$ & -2.88 & -98.58 & 0.01 & 1.15 & -98.42 & -18.65 \\
\hline pear-216 & 34.542826 & -117.905066 & $2,835.9$ & $79,423.93$ & -3.80 & -100.42 & 0.01 & 1.14 & -100.28 & -20.14 \\
\hline pear-217 & 34.542660 & -117.887565 & $2,859.2$ & $79,419.84$ & & -103.10 & 0.01 & 1.14 & -102.96 & -22.42 \\
\hline pear-218 & 34.542660 & -117.870231 & $2,887.4$ & $79,415.80$ & -7.07 & -105.45 & 0.02 & 1.14 & -105.32 & -24.40 \\
\hline pear-219 & 34.542493 & -117.852731 & $2,904.8$ & $79,413.37$ & & -106.82 & 0.02 & 1.14 & -106.70 & -25.41 \\
\hline pear-227 & 34.542494 & -117.765228 & $2,996.3$ & $79,404.27$ & -8.35 & -110.44 & 0.02 & 1.14 & -110.34 & -27.36 \\
\hline pear-228 & 34.542327 & -117.760895 & $3,004.2$ & $79,403.33$ & -8.53 & -110.89 & 0.02 & 1.14 & -110.79 & -27.74 \\
\hline pear-229 & 34.542161 & -117.756561 & $3,012.4$ & $79,402.68$ & -8.40 & -111.04 & 0.02 & 1.14 & -110.94 & -27.81 \\
\hline pear-230 & 34.542161 & -117.752061 & $3,021.4$ & $79,401.90$ & -8.34 & -111.29 & 0.02 & 1.14 & -111.19 & -27.97 \\
\hline pear-231 & 34.541994 & -117.747394 & $3,026.6$ & $79,401.53$ & -8.21 & -111.33 & 0.02 & 1.14 & -111.24 & -27.95 \\
\hline pear-232 & 34.541994 & -117.743228 & $3,035.2$ & $79,400.74$ & -8.19 & -111.60 & 0.02 & 1.13 & -111.52 & -28.15 \\
\hline pear-233 & 34.541994 & -117.738727 & $3,038.8$ & $79,400.29$ & -8.30 & -111.84 & 0.02 & 1.13 & -111.76 & -28.31 \\
\hline pear-234 & 34.542161 & -117.734227 & $3,044.0$ & $79,399.96$ & -8.15 & -111.87 & 0.02 & 1.12 & -111.80 & -28.28 \\
\hline pear-235 & 34.541994 & -117.730727 & $3,047.9$ & $79,399.79$ & -7.94 & -111.79 & 0.03 & 1.13 & -111.72 & -28.15 \\
\hline pear-236 & 34.542327 & -117.726394 & $3,057.5$ & $79,399.12$ & -7.74 & -111.92 & 0.03 & 1.12 & -111.85 & -28.22 \\
\hline pear-237 & 34.542161 & -117.722060 & $3,058.4$ & $79,399.13$ & -7.63 & -111.84 & 0.04 & 1.12 & -111.77 & -28.07 \\
\hline pear-238 & 34.542160 & -117.717893 & $3,074.5$ & $79,397.89$ & -7.36 & -112.11 & 0.03 & 1.11 & -112.06 & -28.29 \\
\hline pear-239 & 34.542160 & -117.713393 & $3,080.4$ & $79,397.64$ & -7.05 & -112.01 & 0.03 & 1.11 & -111.96 & -28.13 \\
\hline pear-240 & 34.542994 & -117.710226 & $3,079.4$ & $79,398.16$ & -6.70 & -111.62 & 0.04 & 1.10 & -111.58 & -27.71 \\
\hline pear-241 & 34.540494 & -117.707059 & $3,103.7$ & $79,397.28$ & -5.08 & -110.83 & 0.68 & 1.76 & -110.13 & -26.21 \\
\hline pear-242 & 34.542160 & -117.703392 & $3,106.3$ & $79,397.46$ & -4.80 & -110.64 & 0.59 & 1.66 & -110.05 & -26.08 \\
\hline pear-243 & 34.541827 & -117.698726 & $3,090.6$ & $79,397.27$ & -6.43 & -111.74 & 0.05 & 1.11 & -111.69 & -27.64 \\
\hline pear-244 & 34.542327 & -117.694559 & $3,087.6$ & $79,396.27$ & -7.76 & -112.96 & 0.02 & 1.07 & -112.95 & -28.83 \\
\hline
\end{tabular}


Table 1. Gravity measurements collected in 2014 near Piñon Hills, California.-Continued

[See fig. 4 for well locations. Extent of inner-terrain correction 2,000 meters. Abbreviations: ft, foot; mGal, milligals; NAD 83, North American Datum of 1983; NAVD 88, North American Vertical Datum of 1988]

\begin{tabular}{|c|c|c|c|c|c|c|c|c|c|c|}
\hline $\begin{array}{c}\text { Station } \\
\text { name }\end{array}$ & $\begin{array}{c}\text { Latitude } \\
\text { (Degrees, } \\
\text { North }\end{array}$ & $\begin{array}{l}\text { Longitude } \\
\text { (Degrees, } \\
\text { West }\end{array}$ & $\begin{array}{l}\text { Land- } \\
\text { surface } \\
\text { altitude } \\
\text { (NAVD 88) }\end{array}$ & $\begin{array}{c}\text { 'Observed } \\
\text { gravity, } \\
\text { minus } \\
900,000\end{array}$ & $\begin{array}{c}\text { Free-air } \\
\text { anomaly } \\
\text { (mGal) }\end{array}$ & $\begin{array}{c}\text { Simple- } \\
\text { Bouguer } \\
\text { anomaly } \\
\text { (mGal) }\end{array}$ & $\begin{array}{c}\text { Inner- } \\
\text { terrain } \\
\text { correction } \\
\text { (mGal) }\end{array}$ & $\begin{array}{c}\text { Total- } \\
\text { terrain } \\
\text { correction } \\
\text { (mGal) }\end{array}$ & $\begin{array}{l}\text { Complete- } \\
\text { Bouguer } \\
\text { gravity field } \\
\text { (mGal) }\end{array}$ & $\begin{array}{c}\text { Isostatic } \\
\text { residual } \\
\text { gravity field } \\
\text { (mGal) }\end{array}$ \\
\hline & & & & (mGal) & \multicolumn{6}{|c|}{ Gravity anomalies and corrections } \\
\hline pear-245 & 34.542327 & -117.690059 & $3,089.5$ & $79,395.35$ & -8.49 & -113.76 & 0.02 & 1.06 & -113.76 & -29.58 \\
\hline pear-246 & 34.542327 & -117.685725 & $3,089.2$ & $79,394.81$ & -9.06 & -114.32 & 0.02 & 1.05 & -114.33 & -30.08 \\
\hline pear-247 & 34.542327 & -117.681225 & $3,101.3$ & $79,393.27$ & -9.46 & -115.14 & 0.01 & 1.04 & -115.16 & -30.85 \\
\hline pear-248 & 34.542493 & -117.663557 & $3,128.9$ & $79,390.16$ & -9.99 & -116.61 & 0.01 & 1.01 & -116.66 & -32.13 \\
\hline pear-249 & 34.542160 & -117.651223 & $3,136.1$ & $79,388.78$ & -10.67 & -117.53 & 0.01 & 1.00 & -117.60 & -32.92 \\
\hline pear-250 & 34.540660 & -117.643390 & $3,154.1$ & $79,387.11$ & -10.52 & -117.99 & 0.01 & 1.00 & -118.07 & -33.28 \\
\hline pear- 251 & 34.542827 & -117.621055 & $3,151.2$ & $79,387.84$ & -10.24 & -117.62 & 0.01 & 0.96 & -117.74 & -32.70 \\
\hline pear-252 & 34.533994 & -117.695892 & $3,131.3$ & $79,392.88$ & -6.34 & -113.03 & 0.03 & 1.16 & -112.94 & -28.83 \\
\hline pear-253 & 34.530661 & -117.695392 & $3,159.2$ & $79,390.37$ & -5.94 & -113.59 & 0.02 & 1.19 & -113.47 & -29.35 \\
\hline pear-254 & 34.526994 & -117.695392 & $3,179.2$ & $79,388.49$ & -5.63 & -113.96 & 0.02 & 1.23 & -113.81 & -29.71 \\
\hline pear-255 & 34.523494 & -117.695392 & $3,201.5$ & $79,386.67$ & -5.06 & -114.15 & 0.02 & 1.28 & -113.96 & -29.89 \\
\hline pear-256 & 34.519828 & -117.695392 & $3,224.1$ & $79,384.82$ & -4.47 & -114.33 & 0.02 & 1.33 & -114.10 & -30.03 \\
\hline pear-257 & 34.516328 & -117.695392 & $3,248.1$ & $79,382.92$ & -3.82 & -114.50 & 0.02 & 1.38 & -114.22 & -30.14 \\
\hline pear- 258 & 34.512828 & -117.695392 & $3,273.3$ & 31.18 & -2.90 & -114.44 & 0.03 & 1.45 & -114.09 & -30.03 \\
\hline pear-259 & 34.509328 & -117.695392 & $3,299.3$ & .39 & -1.95 & -114.37 & & 1. & -113.98 & \\
\hline pear-260 & 34.506328 & -117.695392 & $3,323.9$ & $79,377.80$ & -0.97 & -11 & & 1.55 & -11 & -2 \\
\hline pear-261 & 34.502662 & -117.695058 & $3,355.7$ & $79,375.94$ & 0.47 & -113.88 & 0.04 & 1.62 & -113.38 & -29.35 \\
\hline pear-262 & 34.498829 & -117.695225 & $3,389.5$ & $79,373.74$ & 1.77 & -113.73 & 0.04 & 1.70 & -113.16 & -29.15 \\
\hline pear-263 & 34.495162 & -117.695558 & $3,423.4$ & .52 & 3.04 & -11 & 0.04 & 1.78 & -112.98 & .01 \\
\hline pear-264 & 34.490996 & -117.690892 & $3,468.7$ & 688.50 & 4.6 & -11 & 0 & 1.86 & -112.86 & .84 \\
\hline pear-265 & 34.484329 & -117.804396 & $3,436.2$ & $79,378.02$ & 11.65 & -105.44 & 0.12 & 2.31 & -104.26 & -22.41 \\
\hline pear-266 & 34.484329 & -117.817730 & $3,415.5$ & $79,380.50$ & 12.19 & -104.20 & 0.11 & 2.27 & -103.06 & -21.50 \\
\hline pear-267 & 34.491662 & -117.831397 & $3,321.9$ & $79,386.46$ & 8.74 & -104.46 & 0.07 & 2.03 & -103.54 & -22.17 \\
\hline pear-268 & 34.498662 & -117.811063 & $3,273.7$ & 61 & 4 & -10 & 0 & 1.87 & -106.02 & \\
\hline pear-269 & 34.528326 & -117.931900 & $2,904.5$ & 1.77 & 1. & -97.25 & & 1.35 & -96.91 & -17.48 \\
\hline pear-270 & 34.528327 & -117.914399 & $2,876.3$ & $79,421.49$ & -1.22 & -99.22 & 0.02 & 1.35 & -98.87 & -19.01 \\
\hline pear-271 & 34.529493 & -117.896899 & $2,913.0$ & $79,416.06$ & -3.29 & -102.55 & 0.02 & 1.31 & -102.25 & -22.00 \\
\hline pear-272 & 34.528160 & -117.879398 & $2,959.3$ & $79,410.52$ & -4.37 & -105.20 & 0.02 & 1.32 & -104.91 & -24.26 \\
\hline pear-273 & 34.524161 & -117.853231 & $3,020.3$ & 05.56 & 25 & -106.17 & 002 & 1.37 & -105.84 & -24.66 \\
\hline pear-274 & 34.524161 & -117.835564 & $3,019.4$ & 5.66 & -3.24 & -10 & 0 & 1.38 & -105.78 & -24.22 \\
\hline pear-275 & 34.527827 & -117.817897 & $2,997.4$ & $79,404.94$ & -6.35 & -108.47 & 0.03 & 1.35 & -108.16 & -26.18 \\
\hline pear-276 & 34.525328 & -117.800729 & $3,037.4$ & $79,401.04$ & -6.27 & -109.77 & 0.04 & 1.41 & -109.41 & -27.12 \\
\hline pear-277 & 34.527994 & -117.782729 & $3,054.8$ & $79,399.35$ & -6.55 & -110.64 & 0.04 & 1.36 & -110.33 & -27.67 \\
\hline pear-278 & 34.527161 & -117.778395 & $3,072.9$ & $79,397.93$ & -6.20 & -110.90 & 0.03 & 1.36 & -110.60 & -27.87 \\
\hline pear-279 & 34.527494 & -117.774062 & $3,074.9$ & $79,397.63$ & -6.34 & -111.11 & 00 & 1.36 & -110.81 & -27.99 \\
\hline pear-280 & 34.527828 & -117.769728 & $3,082.1$ & $79,396.98$ & -6.34 & -111.36 & 0.03 & 1.35 & -111.07 & -28.17 \\
\hline pear-281 & 34.528328 & -117.765395 & $3,087.3$ & $79,396.54$ & -6.34 & -111.53 & 0.03 & 1.34 & -111.25 & -28.27 \\
\hline pear-282 & 34.528661 & -117.761062 & $3,091.3$ & $79,396.20$ & -6.33 & -111.66 & 0.03 & 1.33 & -111.39 & -28.34 \\
\hline pear-283 & 34.528995 & -117.756561 & $3,095.8$ & $79,395.88$ & -6.25 & -111.74 & 0.03 & 1.32 & -111.48 & -28.36 \\
\hline pear-284 & 34.529328 & -117.752228 & $3,100.8$ & $79,395.67$ & -6.02 & -111.67 & 0.03 & 1.31 & -111.43 & -28.23 \\
\hline pear-285 & 34.529828 & -117.747728 & $3,101.8$ & 95.67 & -5.97 & -111.66 & 0.03 & 1.30 & -111.42 & -28.14 \\
\hline pear-286 & 34.530161 & -117.743228 & $3,106.7$ & $79,395.39$ & -5.82 & -111.67 & 0.03 & 1.28 & -111.45 & -28.09 \\
\hline pear-287 & 34.530494 & -117.738894 & $3,108.6$ & $79,395.05$ & -6.01 & -111.93 & 0.02 & 1.26 & -111.73 & -28.30 \\
\hline pear-288 & 34.530828 & -117.734560 & $3,110.6$ & $79,394.99$ & -5.91 & -111.89 & 0.03 & 1.26 & -111.70 & -28.19 \\
\hline pear-289 & 34.531161 & -117.730394 & $3,114.2$ & $79,395.24$ & -5.35 & -111.46 & 0.02 & 1.24 & -111.28 & -27.70 \\
\hline pear-290 & 34.531661 & -117.726227 & $3,118.2$ & $79,395.67$ & -4.58 & -110.83 & 0.02 & 1.23 & -110.67 & -27.02 \\
\hline pear-291 & 34.531994 & -117.721727 & $3,121.8$ & $79,394.58$ & -5.36 & -111.73 & 0.02 & 1.22 & -111.58 & -27.86 \\
\hline pear-292 & 34.532328 & -117.717393 & $3,119.8$ & $79,394.48$ & -5.68 & -111.98 & 0.03 & 1.22 & -111.83 & -28.04 \\
\hline pear-293 & 34.531994 & -117.713060 & $3,133.2$ & $79,393.77$ & -5.10 & -111.86 & 0.02 & 1.21 & -111.72 & -27.87 \\
\hline
\end{tabular}


Table 1. Gravity measurements collected in 2014 near Piñon Hills, California.-Continued

[See fig. 4 for well locations. Extent of inner-terrain correction 2,000 meters. Abbreviations: ft, foot; mGal, milligals; NAD 83, North American Datum of 1983; NAVD 88, North American Vertical Datum of 1988]

\begin{tabular}{|c|c|c|c|c|c|c|c|c|c|c|}
\hline $\begin{array}{c}\text { Station } \\
\text { name }\end{array}$ & $\begin{array}{c}\text { Latitude } \\
\text { (Degrees, } \\
\text { North }\end{array}$ & $\begin{array}{c}\text { Longitude } \\
\text { (Degrees, } \\
\text { West }\end{array}$ & $\begin{array}{l}\text { Land- } \\
\text { surface } \\
\text { altitude } \\
\text { (NAVD 88) }\end{array}$ & $\begin{array}{c}\text { 'Observed } \\
\text { gravity, } \\
\text { minus } \\
900,000\end{array}$ & $\begin{array}{c}\text { Free-air } \\
\text { anomaly } \\
\text { (mGal) }\end{array}$ & $\begin{array}{c}\text { Simple- } \\
\text { Bouguer } \\
\text { anomaly } \\
\text { (mGal) }\end{array}$ & $\begin{array}{l}\text { Inner- } \\
\text { terrain } \\
\text { correction } \\
\text { (mGal) }\end{array}$ & $\begin{array}{l}\text { Total- } \\
\text { terrain } \\
\text { correction } \\
\text { (mGal) }\end{array}$ & $\begin{array}{l}\text { Complete- } \\
\text { Bouguer } \\
\text { gravity field g } \\
\text { (mGal) }\end{array}$ & $\begin{array}{c}\text { Isostatic } \\
\text { residual } \\
\text { gravity field } \\
\text { (mGal) }\end{array}$ \\
\hline & & & (ffl) & (mGal) & \multicolumn{6}{|c|}{ Gravity anomalies and corrections } \\
\hline pear-294 & 34.532661 & -117.710226 & $3,137.5$ & $79,395.04$ & -3.48 & -110.39 & 0.06 & 1.23 & -110.22 & -26.34 \\
\hline pear-295 & 34.533327 & -117.705726 & $3,133.9$ & $79,394.11$ & -4.81 & -111.59 & 0.03 & 1.19 & -111.47 & -27.52 \\
\hline pear-296 & 34.533661 & -117.701226 & $3,133.6$ & $79,393.59$ & -5.38 & -112.15 & 0.03 & 1.16 & -112.07 & -28.05 \\
\hline pear-297 & 34.533994 & -117.696892 & $3,131.6$ & $79,393.07$ & -6.12 & -112.82 & 0.03 & 1.17 & -112.73 & -28.64 \\
\hline pear-298 & 34.534494 & -117.692392 & $3,133.6$ & $79,392.19$ & -6.85 & -113.63 & 0.02 & 1.15 & -113.55 & -29.38 \\
\hline pear-299 & 34.534827 & -117.688225 & $3,133.6$ & $79,391.51$ & -7.56 & -114.33 & 0.03 & 1.15 & -114.26 & -30.03 \\
\hline pear-300 & 34.535161 & -117.683725 & $3,135.2$ & $79,390.64$ & -8.31 & -115.14 & 0.03 & 1.14 & -115.07 & -30.79 \\
\hline pear-301 & 34.535494 & -117.679391 & $3,137.2$ & $79,390.16$ & -8.63 & -115.52 & 0.03 & 1.13 & -115.47 & -31.13 \\
\hline pear-302 & 34.535994 & -117.675058 & $3,139.2$ & $79,390.17$ & -8.47 & -115.44 & 0.02 & 1.10 & -115.41 & -31.02 \\
\hline pear-303 & 34.536327 & -117.670724 & $3,144.4$ & $79,389.26$ & -8.92 & -116.06 & 0.02 & 1.09 & -116.05 & -31.60 \\
\hline pear-304 & 34.536660 & -117.666557 & $3,150.0$ & $79,388.64$ & -9.04 & -116.38 & & 1.07 & -11 & \\
\hline pear-305 & 34.536994 & -117.662057 & $3,154.6$ & $79,388.01$ & -9.27 & -116.76 & 0.01 & 1.06 & -116.78 & -32.23 \\
\hline pear-306 & 34.538493 & -117.644223 & $3,160.1$ & $79,386.61$ & -10.27 & -117.95 & 0.01 & 1.02 & -118.01 & -33.22 \\
\hline pear-307 & 34.530160 & -117.625722 & $3,220.8$ & $79,381.77$ & -8.71 & -118.45 & 0.02 & 1.08 & -118.46 & -33.44 \\
\hline pear-308 & 34.528160 & -117.608388 & $3,254.0$ & 8.63 & -8.56 & -119.44 & & 1.0 & -119.46 & \\
\hline pear-309 & 34.531994 & -117.713060 & $3,133.2$ & $79,393.79$ & -5.08 & -111.84 & 0.0 & 1.21 & -11 & -2 \\
\hline pear-310 & 34.528828 & -117.711059 & $3,152.9$ & $79,391.88$ & -4.87 & -112.30 & 0.02 & 1.24 & -112.13 & -28.25 \\
\hline pear-311 & 34.527494 & -117.706893 & $3,166.1$ & $79,390.47$ & -4.93 & -112.81 & 0.02 & 1.25 & -112.64 & -28.70 \\
\hline pear-312 & 34.525661 & -117.703226 & $3,180.5$ & $79,388.85$ & -5.04 & -113.41 & 0.02 & 1.26 & -113.23 & -29.26 \\
\hline pear-313 & 34.523994 & -117.699225 & $3,195.6$ & $79,387.33$ & -5.00 & -113.88 & & 1.28 & -113.69 & -29.66 \\
\hline pear-314 & 34.522494 & -117.695559 & $3,207.7$ & $79,386.08$ & -4.98 & -114.28 & 0.02 & 1.29 & -114.08 & -30.02 \\
\hline pear-315 & 34.520828 & -117.691558 & $3,219.5$ & $79,384.86$ & -4.95 & -114.65 & 0.02 & 1.31 & -114.44 & -30.31 \\
\hline pear-316 & 34.519328 & -117.687892 & $3,233.0$ & $79,383.93$ & -4.49 & -114.65 & 0.02 & 1.32 & -114.42 & -30.24 \\
\hline pear-317 & 34.517828 & -117.684058 & .4 & 79 & -3 & -114.64 & 0 & 1.33 & -114.40 & 16 \\
\hline pear-318 & 34.516161 & -117.680225 & $3,259.2$ & $79,382.09$ & -3.60 & -114.65 & 0. & 1.35 & -114.40 & 11 \\
\hline pear-319 & 34.514661 & -117.676391 & $3,274.7$ & $79,380.63$ & -3.47 & -115.06 & 0.02 & 1.36 & -114.80 & -30.45 \\
\hline pear-320 & 34.512995 & -117.672557 & $3,283.8$ & $79,379.65$ & -3.46 & -115.35 & 0.02 & 1.37 & -115.08 & -30.68 \\
\hline pear-321 & 34.511495 & -117.668724 & $3,294.3$ & $79,378.65$ & -3.34 & -115.59 & 0.02 & 1.38 & -115.32 & -30.87 \\
\hline pear-322 & 34.509828 & -117.664724 & $3,305.8$ & 77.54 & -3 & -115.88 & 0.0 & 1.40 & -115.59 & -31.09 \\
\hline pear-323 & 34.508161 & -117.660724 & $3,315.7$ & $79,376.54$ & -3. & -116.14 & 0 & 1.41 & -115.85 & -31.31 \\
\hline pear-324 & 34.506495 & -117.656723 & $3,326.5$ & $79,375.43$ & -3.11 & -116.46 & 0.02 & 1.42 & -116.16 & -31.57 \\
\hline pear-325 & 34.496495 & -117.643556 & $3,399.0$ & $79,369.40$ & -1.48 & -117.31 & 0.03 & 1.55 & -116.88 & -32.16 \\
\hline pear-326 & 34.521161 & -117.664557 & $3,233.3$ & $79,382.50$ & -6.04 & -116.22 & 0.02 & 1.25 & -116.06 & -31.55 \\
\hline pear-327 & 34.521161 & -117.669057 & $3,232.7$ & $79,382.74$ & -5.86 & -116.01 & 0.02 & 1.26 & -115.85 & -31.39 \\
\hline pear-328 & 34.520161 & -117.673224 & $3,238.2$ & $79,382.63$ & -5.3 & 71 & 0.0 & 1.28 & -115.52 & -31.12 \\
\hline pear-329 & 34.518994 & -117.677558 & $3,245.8$ & $79,382.53$ & -4.65 & -115.25 & 0.02 & 1.30 & -115.05 & -30.71 \\
\hline pear-330 & 34.517828 & -117.681725 & $3,247.4$ & $79,383.25$ & -3.69 & -114.34 & 0.02 & 1.33 & -114.11 & -29.84 \\
\hline pear-331 & 34.517828 & -117.684058 & $3,247.4$ & $79,382.96$ & -3.98 & -114.63 & 0.02 & 1.33 & -114.39 & -30.15 \\
\hline pear-332 & 34.516328 & -117.687892 & $3,253.0$ & $79,382.64$ & -3.64 & -114.49 & 0.02 & 1.36 & -114.22 & -30.04 \\
\hline pear-333 & 34.515161 & -117.692392 & $3,258.2$ & $79,382.08$ & -3.61 & -114.64 & 0.03 & 1.40 & -114.34 & -30.23 \\
\hline pear-334 & 34.513995 & -117.696559 & $3,262.5$ & $79,381.95$ & -3.24 & -114.41 & 0.03 & 1.43 & -114.08 & -30.02 \\
\hline pear-335 & 34.512995 & -117.700559 & $3,268.4$ & $79,381.74$ & -2.81 & -114.18 & 0.03 & 1.45 & -113.83 & -29.84 \\
\hline pear-336 & 34.511995 & -117.704559 & $3,273.0$ & $79,381.66$ & -2.38 & -113.90 & 0.03 & 1.48 & -113.53 & -29.60 \\
\hline pear-337 & 34.510828 & -117.708893 & $3,278.6$ & $79,381.52$ & -1.89 & -113.61 & 0.03 & 1.51 & -113.20 & -29.35 \\
\hline pear-338 & 34.510162 & -117.713726 & $3,282.9$ & $79,381.40$ & -1.55 & -113.42 & 0.04 & 1.54 & -112.98 & -29.20 \\
\hline pear-339 & 34.508495 & -117.717560 & $3,300.6$ & $79,380.31$ & -0.84 & -113.30 & 0.04 & 1.58 & -112.84 & -29.13 \\
\hline pear-340 & 34.507329 & -117.721893 & $3,307.8$ & $79,380.08$ & -0.29 & -113.00 & 0.04 & 1.61 & -112.51 & -28.87 \\
\hline pear-341 & 34.506329 & -117.726227 & $3,318.9$ & $79,379.53$ & 0.29 & -112.80 & 0.05 & 1.64 & -112.28 & -28.73 \\
\hline pear-342 & 34.607991 & -117.837564 & $2,757.4$ & $79,430.19$ & -10.42 & -104.36 & 0.44 & 1.08 & -104.26 & -22.76 \\
\hline
\end{tabular}


Table 1. Gravity measurements collected in 2014 near Piñon Hills, California.-Continued

[See fig. 4 for well locations. Extent of inner-terrain correction 2,000 meters. Abbreviations: ft, foot; mGal, milligals; NAD 83, North American Datum of 1983; NAVD 88, North American Vertical Datum of 1988]

\begin{tabular}{|c|c|c|c|c|c|c|c|c|c|c|}
\hline $\begin{array}{l}\text { Station } \\
\text { name }\end{array}$ & $\begin{array}{l}\text { Latitude } \\
\text { (Degrees, } \\
\text { North }\end{array}$ & $\begin{array}{l}\text { Longitude } \\
\text { (Degrees, } \\
\text { West }\end{array}$ & $\begin{array}{l}\text { Land- } \\
\text { surface } \\
\text { altitude } \\
\text { (NAVD 88) }\end{array}$ & $\begin{array}{c}\text { 'Observed } \\
\text { gravity, } \\
\text { minus } \\
900,000\end{array}$ & $\begin{array}{c}\text { Free-air } \\
\text { anomaly } \\
\text { (mGal) }\end{array}$ & $\begin{array}{c}\text { Simple- } \\
\text { Bouguer } \\
\text { anomaly } \\
\text { (mGal) }\end{array}$ & $\begin{array}{l}\text { Inner- } \\
\text { terrain } \\
\text { correction } \\
\text { (mGal) }\end{array}$ & $\begin{array}{c}\text { Total- } \\
\text { terrain } \\
\text { correction } \\
\text { (mGal) }\end{array}$ & $\begin{array}{c}\text { Complete- } \\
\text { Bouguer } \\
\text { gravity field } \\
\text { (mGal) }\end{array}$ & $\begin{array}{l}\text { Isostatic } \\
\text { residual } \\
\text { gravity field } \\
\text { (mGal) }\end{array}$ \\
\hline & & & & (mGal) & \multicolumn{6}{|c|}{ Gravity anomalies and corrections } \\
\hline pear-343 & 34.603991 & -117.834397 & $2,751.2$ & $79,429.80$ & -11.05 & -104.79 & 0.52 & 1.18 & -104.59 & -23.01 \\
\hline pear-344 & 34.589825 & -117.835731 & $2,754.5$ & $79,428.15$ & -11.20 & -105.05 & 0.04 & 0.78 & -105.25 & -23.63 \\
\hline pear-345 & 34.575325 & -117.835731 & $2,797.5$ & $79,423.41$ & -10.67 & -105.99 & 0.01 & 0.84 & -106.13 & -24.51 \\
\hline pear-346 & 34.560993 & -117.835564 & $2,844.4$ & $79,418.81$ & -9.65 & -106.57 & 0.01 & 0.95 & -106.61 & -24.96 \\
\hline pear-347 & 34.546493 & -117.835564 & $2,896.9$ & $79,413.56$ & -8.74 & -107.45 & 0.01 & 1.10 & -107.36 & -25.71 \\
\hline pear-348 & 34.542327 & -117.835230 & $2,915.0$ & $79,412.19$ & -8.06 & -107.38 & 0.01 & 1.14 & -107.26 & -25.60 \\
\hline pear-349 & 34.524161 & -117.835564 & $3,019.6$ & $79,405.78$ & -3.10 & -105.99 & 0.02 & 1.38 & -105.65 & -24.09 \\
\hline pear-350 & 34.509661 & -117.835564 & $3,131.0$ & $79,398.13$ & 0.94 & -105.75 & 0.04 & 1.63 & -105.19 & -23.72 \\
\hline pear-351 & 34.495162 & -117.835397 & $3,273.0$ & $79,389.96$ & 7.34 & -104.18 & 0.06 & 1.94 & -103.35 & -22.02 \\
\hline pear-352 & 34.480662 & -117.835397 & $3,450.6$ & $79,379.56$ & 14.86 & -102.72 & 0.10 & 2.32 & -101.55 & -20.45 \\
\hline pear-353 & 34.465830 & -117.835397 & $3,731.1$ & $79,363.99$ & 26.91 & -100.24 & 0.36 & 2.94 & -98.50 & -17.76 \\
\hline pear-354 & 34.456997 & -117.835396 & $4,049.0$ & $79,344.44$ & 37.99 & -100.00 & 0.50 & 3.47 & -97.79 & -17.30 \\
\hline pear-355 & 34.512327 & -117.887732 & $3,042.6$ & $79,405.72$ & 0.00 & -103.67 & 0.03 & 1.60 & -103.12 & -22.82 \\
\hline pear-356 & 34.512328 & -117.870398 & $3,097.5$ & $79,401.34$ & 0.77 & -104.77 & 0.03 & 1.57 & -104.26 & -23.56 \\
\hline pear-357 & 34.511994 & -117.853231 & $3,110.0$ & $79,399.65$ & 0.29 & -105.68 & 0.03 & 1.58 & -10 & -2 \\
\hline pear-358 & 34.511995 & -117.835564 & $3,110.3$ & $79,399.27$ & -0.06 & -106.04 & 0.03 & 1.58 & -105.53 & -24.04 \\
\hline pear-359 & 34.511828 & -117.817897 & $3,148.7$ & $79,396.36$ & 0.65 & -106.64 & 0.03 & 1.59 & -106.12 & -24.26 \\
\hline pear-360 & 34.508328 & -117.801063 & $3,211.3$ & $79,390.94$ & 1.41 & -108.01 & 0.04 & 1.67 & -107.43 & 26 \\
\hline pear-361 & 34.507495 & -117.796896 & $3,237.9$ & $79,389.26$ & 2.30 & -108.03 & 0.05 & 1.70 & -107.42 & -25.18 \\
\hline pear-362 & 34.506662 & -117.792896 & $3,270.4$ & $79,386.84$ & 3.01 & -108.43 & 0.05 & 1.71 & -107.82 & -25.51 \\
\hline pear-363 & 34.506162 & -117.788396 & $3,295.7$ & $79,384.95$ & 3.54 & -108.76 & 0.05 & 1.73 & -108.14 & -25.73 \\
\hline pear-364 & 34.506329 & -117.784062 & $3,304.2$ & $79,384.03$ & 3.41 & -109.19 & 0.07 & 1.74 & -108.55 & -26.06 \\
\hline pear-365 & 34.506329 & -117.779729 & $3,296.3$ & 79 & 2.51 & -109.81 & 0.08 & 1.75 & -109.16 & -26.58 \\
\hline pear-366 & 34.506329 & -117.774895 & $3,306.1$ & $79,382.86$ & 2.4 & -110.24 & 0 & 1.75 & -109.61 & -26.94 \\
\hline pear-367 & 34.506329 & -117.770395 & $3,311.7$ & $79,382.50$ & 2.58 & -110.27 & 0.07 & 1.74 & -109.64 & -26.89 \\
\hline pear-368 & 34.506495 & -117.766062 & $3,299.6$ & $79,382.95$ & 1.88 & -110.56 & 0.06 & 1.72 & -109.94 & -27.10 \\
\hline pear-369 & 34.506329 & -117.761728 & 3,297.6 & $79,383.05$ & 1.81 & -110.56 & 0.06 & 1.72 & -109.95 & -27.03 \\
\hline pear-370 & 34.506329 & -117.757228 & 9 & 79 & 1. & -11 & 0. & 1.71 & -109.99 & -2 \\
\hline pear-371 & 34.506329 & -117.752895 & $3,306.1$ & $79,382.57$ & 2.1 & -110.53 & 0.06 & 1.70 & -109.94 & -26.85 \\
\hline pear-372 & 34.506162 & -117.748561 & $3,309.4$ & $79,382.05$ & 1.93 & -110.84 & 0.06 & 1.70 & -110.26 & -27.11 \\
\hline pear-373 & 34.506162 & -117.744228 & $3,313.7$ & $79,381.49$ & 1.77 & -111.14 & 0.05 & 1.68 & -110.58 & -27.36 \\
\hline pear-374 & 34.506162 & -117.739894 & $3,314.0$ & $79,381.04$ & 1.35 & -111.57 & 0.05 & 1.67 & -111.02 & -27.70 \\
\hline pear-375 & 34.506162 & -117.735560 & $3,320.6$ & $79,380.21$ & 114 & -112.01 & 0.05 & 1.67 & -111.46 & -28.07 \\
\hline pear-376 & 34.505662 & -117.730560 & $3,326.2$ & 79.45 & 09 & -112.39 & 0 & 1.66 & -111.84 & -28.36 \\
\hline pear-377 & 34.506329 & -117.726393 & $3,319.6$ & $79,379.50$ & 0.32 & -112.79 & 0.04 & 1.63 & -112.27 & -28.72 \\
\hline pear-378 & 34.505829 & -117.722060 & $3,323.5$ & $79,378.98$ & 0.21 & -113.04 & 0.04 & 1.63 & -112.52 & -28.90 \\
\hline pear-379 & 34.505829 & -117.717560 & $3,324.2$ & $79,378.74$ & 0.04 & -113.24 & 0.04 & 1.62 & -112.73 & -29.03 \\
\hline pear-380 & 34.502662 & -117.713059 & $3,348.8$ & $79,376.82$ & 0.70 & -113.41 & 0.04 & 1.68 & -112.86 & -29.10 \\
\hline pear-381 & 34.505662 & -117.708559 & $3,318.6$ & $79,378.89$ & -0.32 & -113.41 & 0.04 & 1.61 & -112.91 & -29.06 \\
\hline pear-382 & 34.557660 & -117.678058 & $3,045.9$ & $79,397.48$ & -11.76 & -115.54 & 0.01 & 0.91 & -115.68 & -31.39 \\
\hline pear-383 & 34.542327 & -117.681225 & $3,101.0$ & $79,393.29$ & -9.47 & -115.13 & 0.01 & 1.04 & -115.16 & -30.85 \\
\hline pear-384 & 34.539994 & -117.678058 & $3,113.6$ & $79,392.10$ & -9.29 & -115.38 & 0.02 & 1.06 & -115.38 & -31.03 \\
\hline pear-385 & 34.535494 & -117.679558 & $3,136.9$ & $79,390.27$ & -8.55 & -115.43 & 0.03 & 1.13 & -115.38 & -31.05 \\
\hline pear-386 & 34.532161 & -117.677891 & $3,161.5$ & $79,388.32$ & -7.90 & -115.63 & 0.02 & 1.15 & -115.56 & -31.19 \\
\hline pear-387 & 34.528661 & -117.677891 & $3,182.8$ & $79,386.83$ & -7.09 & -115.54 & 0.02 & 1.19 & -115.44 & -31.07 \\
\hline pear-388 & 34.527494 & -117.673724 & $3,192.3$ & $79,385.94$ & -6.99 & -115.77 & 0.02 & 1.19 & -115.66 & -31.24 \\
\hline pear-389 & 34.523828 & -117.673391 & $3,214.0$ & $79,384.41$ & -6.17 & -115.69 & 0.02 & 1.24 & -115.54 & -31.12 \\
\hline pear-390 & 34.520161 & -117.673224 & $3,238.2$ & $79,382.66$ & -5.34 & -115.68 & 0.02 & 1.28 & -115.49 & -31.09 \\
\hline pear-391 & 34.521161 & -117.669057 & $3,232.7$ & $79,382.75$ & -5.85 & -116.00 & 0.02 & 1.26 & -115.84 & -31.38 \\
\hline pear-392 & 34.521161 & -117.664557 & $3,233.0$ & $79,382.49$ & -6.08 & -116.24 & 0.02 & 1.25 & -116.09 & -31.58 \\
\hline
\end{tabular}


Table 1. Gravity measurements collected in 2014 near Piñon Hills, California._-Continued

[See fig. 4 for well locations. Extent of inner-terrain correction 2,000 meters. Abbreviations: ft, foot; mGal, milligals; NAD 83, North American Datum of 1983; NAVD 88, North American Vertical Datum of 1988]

\begin{tabular}{|c|c|c|c|c|c|c|c|c|c|c|}
\hline $\begin{array}{c}\text { Station } \\
\text { name }\end{array}$ & $\begin{array}{l}\text { Latitude } \\
\text { (Degrees, } \\
\text { North }\end{array}$ & $\begin{array}{l}\text { Longitude } \\
\text { (Degrees, } \\
\text { West }\end{array}$ & $\begin{array}{l}\text { Land- } \\
\text { surface } \\
\text { altitude } \\
\text { (NAVD 88) }\end{array}$ & $\begin{array}{l}\text { 1Observed } \\
\text { gravity, } \\
\text { minus } \\
900,000\end{array}$ & $\begin{array}{l}\text { Free-air } \\
\text { anomaly } \\
\text { (mGal) }\end{array}$ & $\begin{array}{c}\text { Simple- } \\
\text { Bouguer } \\
\text { anomaly } \\
\text { (mGal) }\end{array}$ & $\begin{array}{l}\text { Inner- } \\
\text { terrain } \\
\text { correction } \\
\text { (mGal) }\end{array}$ & $\begin{array}{l}\text { Total- } \\
\text { terrain } \\
\text { correction } \\
\text { (mGal) }\end{array}$ & $\begin{array}{l}\text { Complete- } \\
\text { Bouguer } \\
\text { gravity field } \\
\text { (mGal) }\end{array}$ & $\begin{array}{l}\text { Isostatic } \\
\text { residual } \\
\text { gravity field } \\
\text { (mGal) }\end{array}$ \\
\hline & & & $(4)$ & (mGal) & \multicolumn{6}{|c|}{ Gravity anomalies and corrections } \\
\hline pear-393 & 34.517494 & -117.664724 & $3,255.3$ & $79,380.91$ & -5.25 & -116.18 & 0.02 & 1.30 & -115.98 & -31.47 \\
\hline pear-394 & 34.513995 & -117.664557 & $3,279.9$ & $79,379.26$ & -4.30 & -116.06 & 0.02 & 1.34 & -115.82 & -31.31 \\
\hline pear-397 & 34.506328 & -117.664557 & $3,329.8$ & $79,376.00$ & -2.22 & -115.68 & 0.02 & 1.45 & -115.35 & -30.86 \\
\hline pear-398 & 34.502662 & -117.664557 & $3,356.0$ & $79,374.29$ & -1.15 & -115.51 & 0.03 & 1.52 & -115.11 & -30.63 \\
\hline pear-399 & 34.498662 & -117.664057 & $3,388.2$ & $79,372.17$ & 0.09 & -115.37 & 0.03 & 1.58 & -114.91 & -30.46 \\
\hline pear-400 & 34.496995 & -117.660057 & $3,393.1$ & $79,371.56$ & 0.08 & -115.54 & 0.03 & 1.60 & -115.07 & -30.57 \\
\hline pear-401 & 34.493329 & -117.660057 & $3,419.3$ & $79,369.93$ & 1.23 & -115.29 & 0.03 & 1.66 & -114.77 & -30.30 \\
\hline pear-406 & 34.476329 & -117.659890 & $3,569.7$ & $79,359.88$ & 6.74 & -114.90 & 0.05 & 2.05 & -114.03 & -29.71 \\
\hline pear-407 & 34.461997 & -117.659890 & $3,717.0$ & $79,349.47$ & 11.39 & -115.28 & 0.10 & 2.50 & -113.98 & -29.86 \\
\hline pear-408 & 34.447831 & -117.664723 & $3,979.5$ & $79,333.45$ & 21.24 & -114.38 & 0.21 & 3.19 & -112.44 & -28.68 \\
\hline pear-409 & 34.430831 & -117.670223 & $4,437.7$ & $79,307.27$ & 39.55 & -111.69 & 0.38 & 4.38 & -108.63 & -25.45 \\
\hline pear-410 & 34.473163 & -117.809063 & $3,637.3$ & $79,367.24$ & 20.72 & -103.23 & 0.21 & 2.69 & -101.72 & -20.19 \\
\hline pear-411 & 34.460330 & -117.800229 & $4,017.6$ & $79,342.53$ & 32.84 & -104.07 & 0.35 & 3.27 & -102.07 & -20.68 \\
\hline pear-412 & 34.472163 & -117.791395 & $3,716.4$ & $79,358.88$ & 19.88 & -106.76 & 0.24 & 2.79 & -105.17 & -23.31 \\
\hline
\end{tabular}

${ }^{1}$ International Gravity Standardization Net 1971 (Morelli, 1974; Hinze and others, 2005).

Table 2. Data for wells with depth information used to constrain the gravity model near Piñon Hills, California.

[See fig. 4 for well locations. Well location estimated to center of Public Land Survey System quarter-quarter section unless otherwise noted. Abbreviations: API, American Petroleum Institute; ft, foot; LARSE, Los Angeles Regional Seismic Experiment; NAD 83, North American Datum of 1983, depths are in feet below land surface; ${ }^{\circ}$, degree]

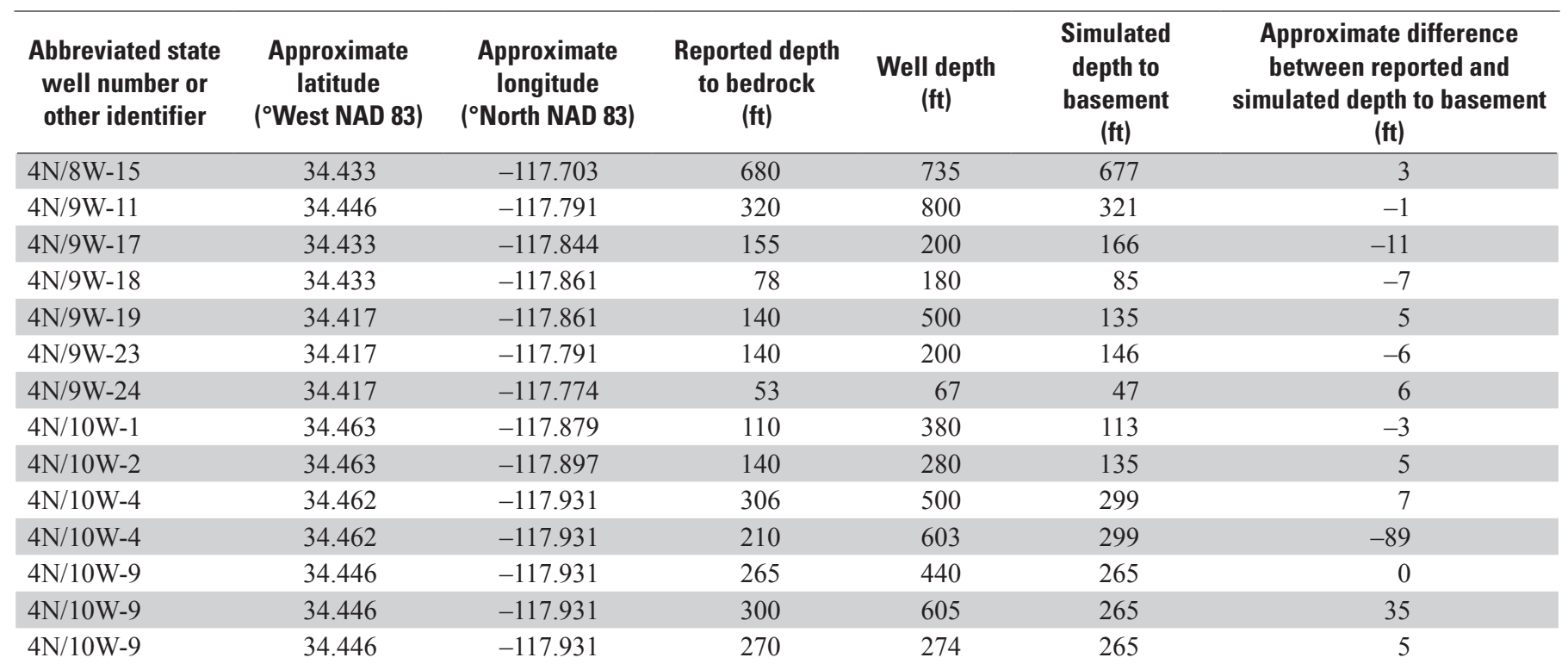


Table 2. Data for wells with depth information used to constrain the gravity model near Piñon Hills, California.-Continued

[See fig. 4 for well locations. Well location estimated to center of Public Land Survey System quarter-quarter section unless otherwise noted. Abbreviations: API, American Petroleum Institute; ft, foot; LARSE, Los Angeles Regional Seismic Experiment; NAD 83, North American Datum of 1983, depths are in feet below land surface; ${ }^{\circ}$, degree]

\begin{tabular}{|c|c|c|c|c|c|c|}
\hline $\begin{array}{l}\text { Abbreviated state } \\
\text { well number or } \\
\text { other identifier }\end{array}$ & $\begin{array}{c}\text { Approximate } \\
\text { latitude } \\
\text { ('West NAD 83) }\end{array}$ & $\begin{array}{c}\text { Approximate } \\
\text { longitude } \\
\text { ('North NAD 83) }\end{array}$ & $\begin{array}{l}\text { Reported depth } \\
\text { to bedrock } \\
\text { (ft) }\end{array}$ & $\begin{array}{l}\text { Well depth } \\
\text { (ft) }\end{array}$ & $\begin{array}{l}\text { Simulated } \\
\text { depth to } \\
\text { basement } \\
\text { (ft) }\end{array}$ & $\begin{array}{c}\text { Approximate difference } \\
\text { between reported and } \\
\text { simulated depth to basement } \\
\text { (ft) }\end{array}$ \\
\hline $4 \mathrm{~N} / 10 \mathrm{~W}-9$ & 34.446 & -117.931 & 70 & 145 & 265 & -195 \\
\hline $4 \mathrm{~N} / 10 \mathrm{~W}-11$ & 34.448 & -117.897 & 80 & 250 & 56 & 24 \\
\hline $4 \mathrm{~N} / 10 \mathrm{~W}-13$ & 34.434 & -117.879 & 457 & 460 & 431 & 26 \\
\hline $4 \mathrm{~N} / 10 \mathrm{~W}-14$ & 34.434 & -117.896 & 175 & 220 & 185 & -10 \\
\hline $4 \mathrm{~N} / 10 \mathrm{~W}-14$ & 34.434 & -117.896 & 100 & 300 & 185 & -85 \\
\hline $4 \mathrm{~N} / 10 \mathrm{~W}-15$ & 34.434 & -117.914 & 240 & 400 & 250 & -10 \\
\hline $5 \mathrm{~N} / 8 \mathrm{~W}-18$ & 34.520 & -117.757 & 520 & 523 & 524 & -4 \\
\hline $5 \mathrm{~N} / 8 \mathrm{~W}-26$ & 34.491 & -117.686 & 985 & 1,013 & 993 & -8 \\
\hline $5 \mathrm{~N} / 8 \mathrm{~W}-26$ & 34.491 & -117.686 & 985 & 1,000 & 993 & -8 \\
\hline $5 \mathrm{~N} / 9 \mathrm{~W}-9$ & 34.535 & -117.827 & 340 & 465 & 346 & -6 \\
\hline $5 \mathrm{~N} / 9 \mathrm{~W}-26$ & 34.491 & -117.791 & 522 & 612 & 524 & -2 \\
\hline $5 \mathrm{~N} / 10 \mathrm{~W}-4 \mathrm{Z}^{1}$ & 34.514 & -117.904 & 610 & 700 & 602 & 8 \\
\hline $5 \mathrm{~N} / 10 \mathrm{~W}-7 \mathrm{~L}^{1}$ & 34.520 & -117.949 & 330 & 370 & 338 & -8 \\
\hline $5 \mathrm{~N} / 10 \mathrm{~W}-7 \mathrm{E} 1^{1}$ & 34.536 & -117.973 & 520 & 550 & 525 & -5 \\
\hline $5 \mathrm{~N} / 10 \mathrm{~W}-7 \mathrm{P} 1^{1}$ & 34.529 & -117.969 & 610 & 625 & 605 & 5 \\
\hline $5 \mathrm{~N} / 10 \mathrm{~W}-7 \mathrm{R} 1^{1}$ & 34.529 & -117.958 & 530 & 550 & 529 & 1 \\
\hline $5 \mathrm{~N} / 10 \mathrm{~W}-9$ & 34.536 & -117.932 & 557 & 571 & 550 & 7 \\
\hline $5 \mathrm{~N} / 10 \mathrm{~W}-10 \mathrm{R}^{1}$ & 34.543 & -117.940 & 412 & 435 & 426 & -14 \\
\hline $5 \mathrm{~N} / 10 \mathrm{~W}-21$ & 34.506 & -117.932 & 120 & 275 & 127 & -7 \\
\hline $5 \mathrm{~N} / 10 \mathrm{~W}-23$ & 34.506 & -117.897 & 375 & 490 & 387 & -12 \\
\hline $5 \mathrm{~N} / 10 \mathrm{~W}-28$ & 34.492 & -117.932 & 140 & 295 & 124 & 16 \\
\hline $5 \mathrm{~N} / 10 \mathrm{~W}-33$ & 34.477 & -117.932 & 300 & 700 & 317 & -17 \\
\hline $5 \mathrm{~N} / 10 \mathrm{~W}-35$ & 34.477 & -117.897 & 85 & 600 & 26 & 59 \\
\hline $5 \mathrm{~N} / 11 \mathrm{~W}-1 \mathrm{M} 1^{1}$ & 34.550 & -117.991 & 380 & 414 & 371 & 9 \\
\hline $5 \mathrm{~N} / 11 \mathrm{~W}-13 \mathrm{G}^{1}$ & 34.521 & -117.979 & 360 & 380 & 338 & 22 \\
\hline $5 \mathrm{~N} / 11 \mathrm{~W}-13 \mathrm{~J}^{1}$ & 34.522 & -117.974 & 366 & 377 & 367 & -1 \\
\hline $6 \mathrm{~N} / 8 \mathrm{~W}-21$ & 34.594 & -117.729 & 150 & 200 & 152 & -2 \\
\hline $6 \mathrm{~N} / 8 \mathrm{~W}-23$ & 34.594 & -117.694 & 180 & 210 & 179 & 1 \\
\hline $6 \mathrm{~N} / 8 \mathrm{~W}-23$ & 34.594 & -117.694 & 160 & 300 & 179 & -19 \\
\hline $6 \mathrm{~N} / 8 \mathrm{~W}-26$ & 34.580 & -117.694 & 516 & 531 & 515 & 1 \\
\hline $6 \mathrm{~N} / 8 \mathrm{~W}-26$ & 34.580 & -117.694 & 460 & 464 & 515 & -55 \\
\hline $6 \mathrm{~N} / 8 \mathrm{~W}-26 \mathrm{P}^{1}$ & 34.571 & -117.694 & 548 & 550 & 548 & 0 \\
\hline $6 \mathrm{~N} / 8 \mathrm{~W}-30$ & 34.580 & -117.765 & 346 & 390 & 343 & 3 \\
\hline $6 \mathrm{~N} / 8 \mathrm{~W}-31$ & 34.565 & -117.765 & 320 & 430 & 316 & 4 \\
\hline $6 \mathrm{~N} / 8 \mathrm{~W}-35$ & 34.565 & -117.694 & 575 & 730 & 570 & 5 \\
\hline $6 \mathrm{~N} / 8 \mathrm{~W}-35$ & 34.565 & -117.694 & 663 & 705 & 570 & 93 \\
\hline $6 \mathrm{~N} / 9 \mathrm{~W}-23$ & 34.595 & -117.800 & 148 & 150 & 101 & 47 \\
\hline $6 \mathrm{~N} / 9 \mathrm{~W}-25$ & 34.580 & -117.782 & 246 & 285 & 248 & -2 \\
\hline $6 \mathrm{~N} / 9 \mathrm{~W}-33$ & 34.565 & -117.835 & 722 & 725 & 713 & 9 \\
\hline $6 \mathrm{~N} / 10 \mathrm{~W}-25$ & 34.580 & -117.890 & 241 & 242 & 243 & -2 \\
\hline $6 \mathrm{~N} / 10 \mathrm{~W}-33$ & 34.565 & -117.943 & 165 & 200 & 165 & 0 \\
\hline API $07100013^{1,2}$ & 34.414 & -117.586 & 3,700 & 6,365 & 3,710 & -10 \\
\hline LARSE $^{3}$ & 34.489 & -117.705 & 1,800 & 4,600 & 1,802 & -2 \\
\hline
\end{tabular}

${ }^{1}$ Location from drillers' log.

${ }^{2}$ Location from California Department of Conservation, DOGGR (2016).

${ }^{3}$ Location from Fuis and others (2001). 


\section{Gravity Field Results}

After correcting for factors not related to upper crustal structure, the isostatic residual gravity field of the study area mostly reflects the large density contrast between the basement complex and lower density basin-fill deposits (fig. 4). The most prominent features on the gravity map are (1) high gravity values (greater than $-15 \mathrm{mGal}$ ) that generally coincide with thin alluvium and basement-complex exposures in the San Gabriel Mountains to the southwest of the San Andreas Fault Zone and (2) low gravity values (less than $-30 \mathrm{mGal}$ ) in the southeastern and northwestern parts of the study area. A local gravity high (shown in light green hues in fig. 4), between Pearblossom, Calif., and the Alpine and Love Joy Buttes, is consistent with the location of exposed or thinly covered basement rocks.

\section{Groundwater Basin Thickness Model}

\section{Methods}

The thickness of the basin-fill deposits (or depth to the basement complex) in the study area was estimated using the method of Jachens and Moring (1990) and Saltus and Jachens (1995), modified slightly to permit inclusion of constraints at points where the thickness was known from drillers' logs. This method separates the isostatic residual gravity field into two components - the component caused by density variations within the basement rocks (the basement gravity field) and the component caused by variations in the thickness of lowdensity basin-fill deposits (basin-fill deposits gravity field). Once the basin-fill gravity field has been isolated, the basin-fill deposits gravity field can be modeled to yield a thickness of the deposits that fill the groundwater basin.

An initial estimate of the basin-fill deposits gravity field is made by fitting a smooth surface to the gravity values measured at stations where the basement-complex rocks are exposed at land surface (initial estimate of the basement gravity field) and subtracting the result from the total gravity field. This resulting basin-fill deposits gravity field is only an initial estimate because the gravity field measured on the exposed basement complex adjacent to the alluvial deposits is affected by the adjacent lower-density basin-fill deposits and is therefore lower than it would be if the basin-fill deposits were not present. To compensate for this effect, the initial estimate of the basin-fill deposits gravity field is used to calculate an initial estimate of the thickness of the basin-fill deposits, and the gravity effect of these basin-fill deposits is calculated at all of the basement gravity stations. A second estimate of the basement gravity field is then made by fitting a smooth surface through the adjusted basement gravity values; then a second estimate of the thickness of the alluvial deposits is made. This process was repeated to produce refined estimates of the thickness of the alluvial deposits until further changes to the calculated thickness of the alluvial deposits were minimal.

During the iterative modeling process, the basin-fill deposits gravity field was converted to thickness of the basinfill deposits by using an estimated density contrast between the basin-fill deposits and the basement rocks that varied with depth below land surface (table 3). The density-depth relation used was from Saltus and Jachens (1995). The density contrast of $-650 \mathrm{~kg} / \mathrm{m}^{3}\left(40.57 \mathrm{lb} / \mathrm{ft}^{3}\right)$ used for the basin-fill deposits in the upper $660 \mathrm{ft}$ is reasonable for continental deposits of Quaternary and Tertiary age overlying pre-Tertiary crystalline rocks (Langenheim and others, 2005; Jachens and others, 2014). This value is consistent with the results from other gravity analyses in southern California (Langenheim and others, 2005; Jachens and others, 2014). In those studies, the density contrasts of the basin-fill deposits in the upper $660 \mathrm{ft}$ were -550 and $-590 \mathrm{~kg} / \mathrm{m}^{3}$ ( 34.34 and $36.83 \mathrm{lb} / \mathrm{ft}^{3}$ ) relative to a basement density of $2,670 \mathrm{~kg} / \mathrm{m}^{3}\left(166.68 \mathrm{lb} / \mathrm{ft}^{3}\right)$, lower than the value used in this study. If a lower density contrast is used, the resulting estimated thickness of basin-fill deposits would be greater. The estimate of density contrast $\left(-650 \mathrm{~kg} / \mathrm{m}^{3}\right.$, $40.57 \mathrm{lb} / \mathrm{ft}^{3}$ ) was further evaluated by examining the modeled basement gravity field for any indications of unusual local gravity field at sites where drillers' logs report materials consistent with basement, and the thickness solution was forced to honor a best fit to the observations of depth to basement from the drillers' logs. Because of inconsistent reporting and interpretation of $\log$ data, in some cases, where fitting data from drillers' log caused an unreasonable local gravity field, the log data and model were reviewed and a determination was made as to the use of the log data.

Table 3. Estimated density contrast varied with depth near Piñon Hills, California.

[From density-depth relation used by Saltus and Jachens, 1995. Abbreviations: bls, below land surface; ft, feet; kg, kilogram; $\mathrm{m}^{3}$, cubic meter; >, greater than]

\begin{tabular}{cc}
\hline $\begin{array}{c}\text { Depth range } \\
\text { (ft bls) }\end{array}$ & $\begin{array}{c}\text { Density constrast } \\
\left(\mathbf{k g} / \mathbf{m}^{3}\right)\end{array}$ \\
\hline $0-660$ & -650 \\
$661-2,000$ & -550 \\
$2,001-4,000$ & -350 \\
$>4,000$ & -250 \\
\hline
\end{tabular}

\section{Results of Gravity Thickness Modeling}

The gravity and basin-thickness modeling allowed insights to both the basin-fill deposits and the underlying surface and structure of the basement complex. In the following subsections, the thickness and structure of the basin-fill deposits are first described, followed by the configuration of the top of the basement complex. 


\section{Thickness of the Basin-Fill Deposits}

The calculated thickness of the basin-fill deposits, or depth to basement complex, ranged from $0 \mathrm{ft}$ in the San Gabriel Mountains along the San Andreas Fault Zone and exposed buttes in the study area (shown as less than $250 \mathrm{ft}$ on fig. 5) to about 5,775 $\mathrm{ft}$ in the northwestern part of the study area. The porosity and permeability of the basin-fill deposits decrease with depth because of compaction and cementation. Therefore, although the depth to basement complex is greatest in the northwestern and southeastern parts of the study area, the thickness of permeable water-bearing deposits likely is much less than the total calculated thickness of basin-fill deposits. Compared to the alluvial thickness estimated from Jachens and others (2014), the results of this study showed increased detail in the basement geometry and estimated alluvial thickness. The results from this study provide better resolution of the basement geometry in the vicinity of the subsurface basement ridge suggested by Jachens and others (2014; fig. 3). In addition, the greater detail of the subsurface structure southeast of Three Sisters Buttes revealed an apparent buried butte-like structure beneath well 5N/8W-26B2 (figs. 5, 6), which could be evidence of the southeast extension of the Dibblee Buttes Fault.

The estimated thickness of the basin-fill deposits, or simulated depth to basement, where drill holes have penetrated the entire thickness of the basin-fill deposits agreed with the observed thickness (table 2) within an average of $18 \mathrm{ft}$, which was expected because the solution was constrained by these values. The basin-fill thickness was estimated for grid cells that were approximately $1,000 \mathrm{ft}$ by $1,000 \mathrm{ft}$ (about 23 acres in area); the results represented the average thickness of basin-fill deposits for each cell. Variations in thickness of the basin-fill deposits over distances of less than approximately $1,000 \mathrm{ft}$ were not resolved. In addition, basic uncertainties in the gravity data imply that the best resolution of vertical thickness that can be expected, even in areas of good gravity coverage, is about 50-100 ft; the resolution is likely to be less in areas of poor gravity coverage and in areas far from either the basement outcrop or wells that encountered the basement rocks. Gravity data reflect the average shape of the basin-fill deposits, and the averaging has a greater effect where the basin-fill deposits are thicker (deeper basement surface) and therefore further from the source of the gravity station at land surface. As a result, places where the basin-fill deposits are thickest are subject to averaging over a greater volume of material; thus, thickness contours can appear smoother in these areas than in areas where the basin-fill deposits are thinner. Finally, the model assumes no lateral variations in density within the basin-fill deposits, which may not be representative if the deposits coarsen toward the mountain front.

\section{Structure of the Groundwater Basin}

The basin-fill thickness model was used to calculate the approximate altitude of the basement complex (fig. 6). The altitude of the basement was calculated by subtracting the nodal value of gravity-derived thickness from the landsurface altitude, which was determined from a 10-meter digital elevation model (U.S. Geological Survey, 2009) at the location of the node. These data were used to generate the altitude of basement grid; zero values are at the vertical datum (and roughly represent mean sea level), and negative values represent areas that are below sea level. The model indicated two basement depressions in the northwestern and southeastern parts of the study area (fig. 6). The two basement depressions are separated by a concealed basement high, or ridge of highest basement altitude (shown as yellow line in fig. 6), that trends generally northward from crop outs to the southeast of Llano, Calif., and is present through Three Sisters Buttes and Adobe Mountain. The crest of the subsurface basement ridge is about $1.8 \mathrm{mi}$ north of State Route 138 (SR-138), between wells 5N/8W-18N1 and 5N/8W-20P1.

Dibblee (1959) mapped an unnamed fault that trends southeast and northwest from the Three Sisters Buttes past Saddleback Butte and northwestward. Dibblee (1959) also mapped the general motion of the fault with the east side up and the west side down. As suggested previously, the trend of the mapped surface expression of the Dibblee Buttes Fault generally is in alignment with a subsurface basement ridge located to the southwest of the Three Sister Buttes (figs. 3, 6). Based on the isostatic residual gravity field and thickness model, it could be reasonable to extend the Dibblee Buttes Fault to the southeast along the strike of the fault; the change in course of Mescal Creek just north of SR-18 could be a result from movement on the fault. From its source at the mountain front, Mescal Creek trends generally south to north until about $1 \mathrm{mi}$ beyond SR-18, where it veers to the northwest and follows the strike of the Dibblee Buttes Fault (fig. 2). Although gravity data and geomorphic evidence suggest that the Dibblee Buttes Fault could extend to the southeast toward the San Andreas Fault Zone, it is not certain if this fault is a barrier to groundwater flow. More direct hydrologic data, such as groundwater levels and drillers' logs obtained along the assumed fault trace, could help determine if the Dibblee Buttes Fault, or other faults and their southeast extensions, act as possible hydrologic barriers. 


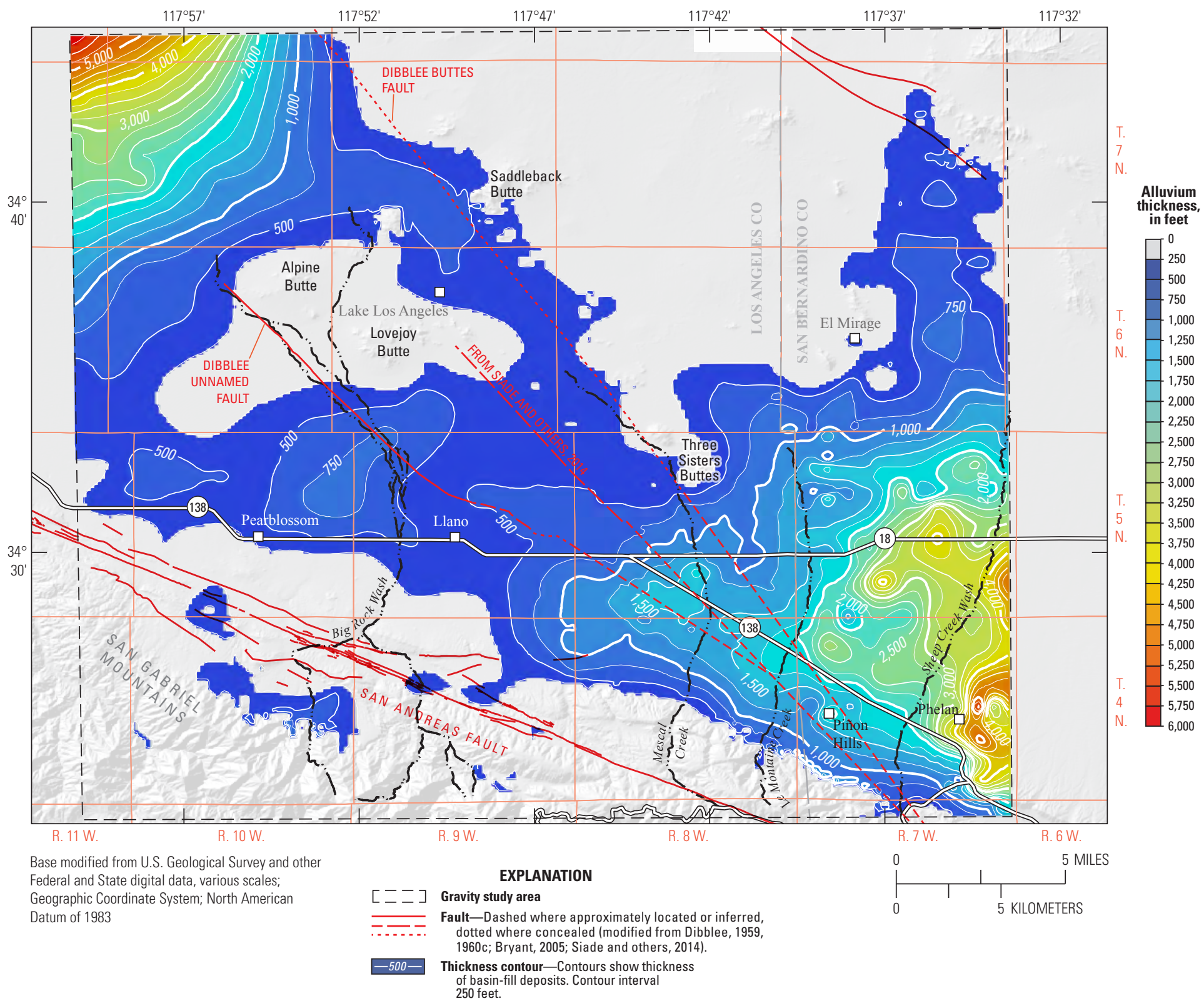

Figure 5. Estimated thickness of basin-fill deposits based on modeled gravity field, near Piñon Hills, California. 


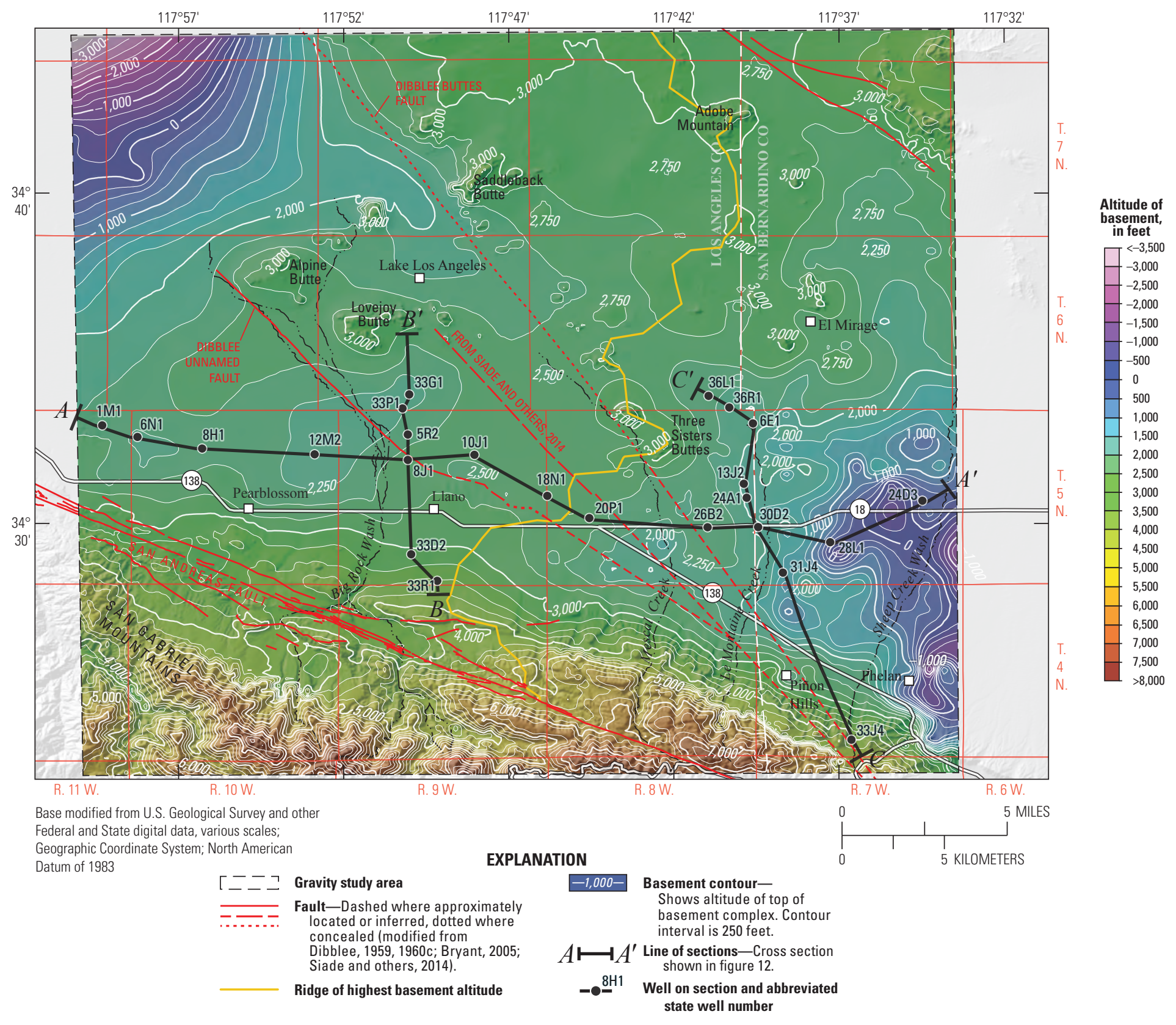

Figure 6. Approximate altitude of basement complex from modeled gravity data and location of hydrogeologic sections and wells on section shown in figure 12, near Piñon Hills, California. 


\section{Groundwater-Level Survey}

Wells and water works have been described near the earliest settlements in the Antelope Valley groundwater basin and Mojave Basin Area since the early 1890s (Johnson, 1911; Thompson, 1929; Dutcher and Worts, 1963). The first documented wells were artesian, or hand-dug, at sources of shallow groundwater near towns. The shallower parts of the younger alluvium, which initially were the principal source of groundwater supply, had groundwater-level declines by the 1920s near the Mojave River (fig. 1) following several years of drought (Thompson, 1929) and had been substantially dewatered in many parts of the Antelope Valley by 1960 (Dutcher and Worts, 1963). Although groundwater use and widespread development have increased near the more populated areas over time, few wells have been drilled and inventoried in rural areas.

\section{Assessment of Existing Groundwater-Level Data}

For this study, groundwater-level data were compiled and collected for an area of about $270 \mathrm{mi}^{2}$. An initial search of existing groundwater-level records for the area at the time of this study (2014) found groundwater-level data available for 87 wells (this number will be different with the subsequent increase in sites with available data since 2014; fig. 7).

Before deep municipal supply wells were installed near the Los Angeles-San Bernardino County line starting in the 1990s, groundwater levels from existing wells were measured and recorded sporadically since 1917 (fig. 8). Before 1940, groundwater levels were measured in only two wells in 1917, three in 1918, and one in 1928. Wells with multiple groundwater-level measurements are uncommon; about 80 percent of the wells have only one or two measurements total.

\section{Results of Field Reconnaissance Survey}

A field-reconnaissance survey was completed in September 2014 to compile existing well location information and to collect new groundwater-level data to determine, if possible, the depth to the water table and direction of groundwater flow. Field reconnaissance was concentrated mainly in the areas where the gravity data showed a potential basement high (fig. 6) and between El Mirage and Big Rock Washes, where recent and historical groundwater-level data were scarce (fig. 9). Because only limited historical well records exist in the area, all potential sites were investigated.

Groundwater-level data were collected from 19 wells during the initial field survey in September 2014 (table 4). To supplement the groundwater-level data collected in September 2014, measurements taken in wells from March 2014 through March 2015 were included in the dataset to construct a groundwater-level map (fig. 10). Reported groundwater-level data from the Phelan-Piñon Hills Community Services District (G. Cardenas, Phelan-Piñon Hills Community Services District, written commun., 2015) municipal supply wells (table 4), near the Los Angeles-San Bernardino County line, were also used.

In general, the direction of groundwater flow indicated from the well data was from areas of recharge along the southern mountain front, where the groundwater-level altitude was greater than $4,600 \mathrm{ft}$, to the northwestern part of the study area, where groundwater-level altitude was about $2,750 \mathrm{ft}$ (fig. 10). The direction of groundwater flow in the eastern part of the study area is south to north and subparallel to the boundary of the Antelope Valley and El Mirage Valley groundwater basins; in the western part of the study area, groundwater flow is northwesterly. This suggests that some groundwater flow could occur between the Antelope Valley and El Mirage Valley groundwater basins, but additional data, discussed in the "Limitations and Considerations for Future Studies" section, are needed to determine vertical and lateral hydraulic gradients.

Pumping from municipal wells along the Los AngelesSan Bernardino County line has caused a localized groundwater-level depression but does not appear to have changed the general northward direction of groundwater flow. Future patterns of groundwater flow likely will be influenced by changes in the number and distribution of pumping wells, the amount of pumpage withdrawn from the aquifer system, and the thickness of the saturated alluvium from which water is withdrawn.

Offsets in the groundwater-level contours on opposite sides of the Dibblee Unnamed Fault and the fault from Siade and others (2014) in the northwestern part of the study area suggest that the faults have some effect on groundwater flow (fig. 10). Generally, groundwater levels in the northwestern part of the study area indicate that groundwater flows out of the study area to the northwest, following the course of the washes.

\section{Long-Term Trends in Groundwater-Level Altitudes}

Groundwater levels from individual wells with multiple measurements were evaluated to determine long-term trends in the data (fig. 11). These long-term data are shown to help evaluate how groundwater-level altitudes have changed over time. Data from wells in close proximity were combined on some hydrographs to show trends over longer time periods. Ten long-term hydrographs are shown for wells with groundwater-level data ranging from 1982 to 2015 (unshaded, fig. 11A); eleven longer-term hydrographs are shown for wells with groundwater-level data ranging from the early 1950s to 2015 (shaded, fig. 11B); data for one well (5N/7W-6E1) ranged from 1918 to 2014 . 


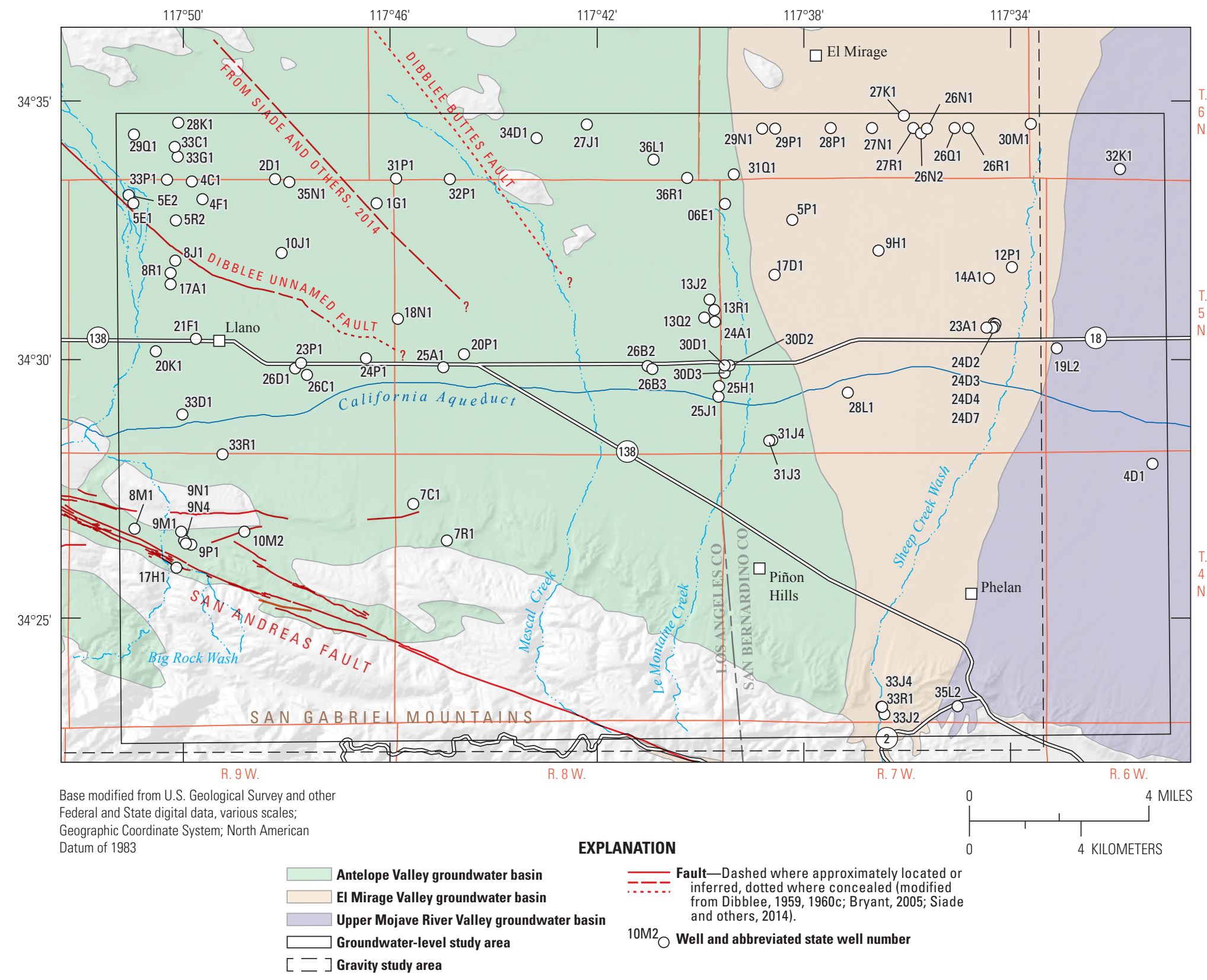

Figure 7. Location of wells with groundwater-level measurements available in 2014, for 1917-2014, near Piñon Hills, California. 


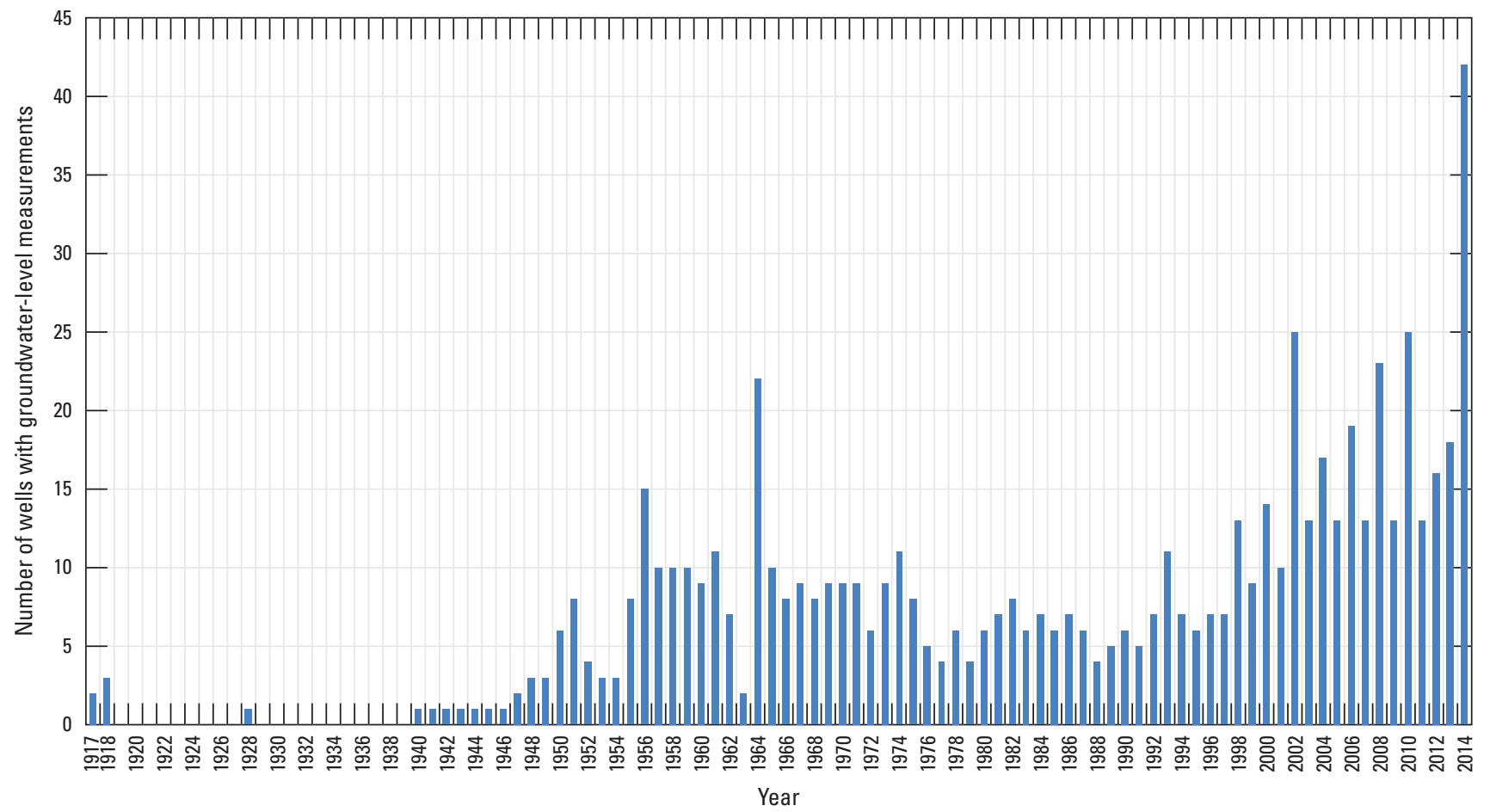

Figure 8. Number of wells with recorded groundwater-level measurements, 1917-2014, near Piñon Hills, California. 


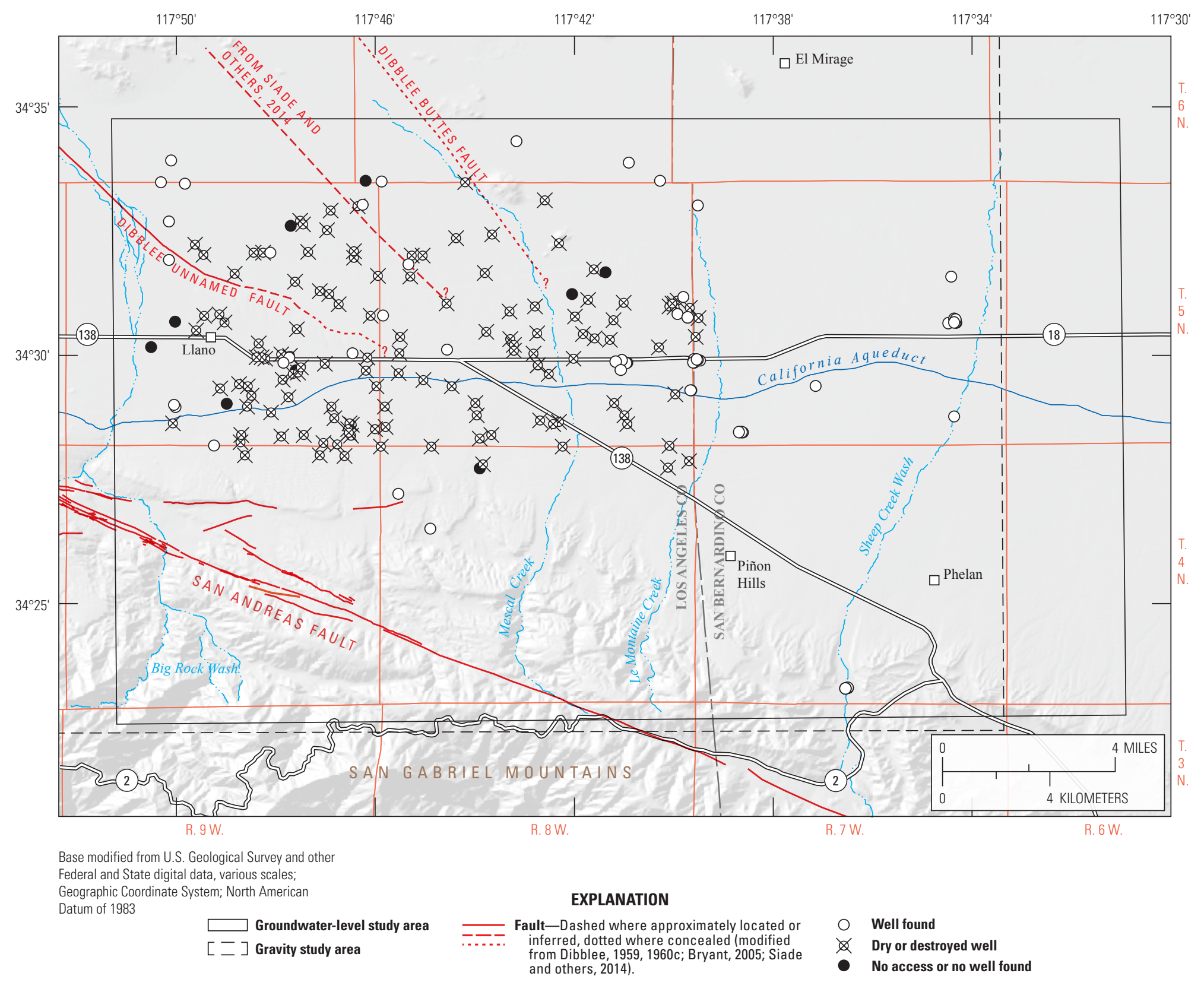

Figure 9. Potential water-level measurement well sites visited during September 2014, near Piñon Hills, California. 
Table 4. Selected wells with groundwater-level data, 1990-2015, near Piñon Hills, California.

[Depths are below land surface. Altitudes are referenced to the North American Vertical Datum of 1988. See figure 10 for groundwater-level map; see figure 11 for hydrographs; see figure 12 for cross sections. Abbreviations: ft, foot; mm/dd/yyyy, month/day/year; UK, unknown; —, not applicable]

\begin{tabular}{|c|c|c|c|c|c|c|c|}
\hline $\begin{array}{c}\text { Abbreviated } \\
\text { state well } \\
\text { number }\end{array}$ & $\begin{array}{c}\text { USGS } \\
\text { site number }\end{array}$ & $\begin{array}{l}\text { Approximate } \\
\text { land- } \\
\text { surface } \\
\text { altitude, } \\
\text { in feet }\end{array}$ & $\begin{array}{c}\text { Date of } \\
\text { static water } \\
\text { level } \\
\text { (mm/dd/yyyy) }\end{array}$ & $\begin{array}{c}\text { Water- } \\
\text { level } \\
\text { altitude, } \\
\text { in feet }\end{array}$ & $\begin{array}{c}\text { Depth to } \\
\text { water, } \\
\text { in feet }\end{array}$ & $\begin{array}{l}\text { Well } \\
\text { depth, } \\
\text { in feet }\end{array}$ & Use of water-level data \\
\hline $4 \mathrm{~N} / 6 \mathrm{~W}-4 \mathrm{D} 2^{1}$ & 342800117310001 & 3,583 & - & - & - & 1,130 & Hydrograph. \\
\hline $4 \mathrm{~N} / 7 \mathrm{~W}-33 \mathrm{~J} 2^{1}$ & 342318117362901 & 4,879 & - & - & - & UK & Hydrograph. \\
\hline 4N/8W-7C1 & 342713117453001 & 4,217 & $03 / 04 / 2015$ & 3,976 & 240.52 & 280 & Hydrograph, water-level map. \\
\hline 4N/8W-7R1 & 342631117445101 & 4,310 & $03 / 06 / 2015$ & 4,191 & 118.84 & 185 & Hydrograph, water-level map. \\
\hline $5 \mathrm{~N} / 7 \mathrm{~W}-6 \mathrm{E} 1$ & 343308117392801 & 3,106 & 09/08/2014 & ${ }^{2} 2,872$ & 234.03 & UK & Hydrograph, cross section, water-level map. \\
\hline $5 \mathrm{~N} / 7 \mathrm{~W}-9 \mathrm{H} 1$ & 343207117363001 & 3,214 & - & - & - & UK & Hydrograph. \\
\hline $5 \mathrm{~N} / 7 \mathrm{~W}-24 \mathrm{D} 3^{1}$ & 343038117341701 & 3,348 & $09 / 15 / 2014$ & 2,916 & 432 & 660 & Cross section, water-level map. \\
\hline $5 \mathrm{~N} / 7 \mathrm{~W}-24 \mathrm{D} 7^{1}$ & 343043117342001 & 3,340 & 03/06/2014 & 2,923 & 416.79 & UK & Water-level map. \\
\hline $5 \mathrm{~N} / 7 \mathrm{~W}-28 \mathrm{~L} 1$ & 342923117370601 & 3,504 & 09/03/2014 & 2,951 & 553.16 & 626 & Hydrograph, cross section, water-level map. \\
\hline $5 \mathrm{~N} / 7 \mathrm{~W}-30 \mathrm{D} 1^{1}$ & 342954117392601 & 3,388 & $12 / 03 / 2014$ & 2,908 & 480 & 825 & Water-level map, cross section. \\
\hline $5 \mathrm{~N} / 7 \mathrm{~W}-30 \mathrm{D} 2^{1}$ & 343002117393301 & 3,385 & $12 / 01 / 2014$ & 2,899 & 485.7 & 795 & Hydrograph, water-level map. \\
\hline $5 \mathrm{~N} / 7 \mathrm{~W}-30 \mathrm{D} 3^{1}$ & 342951117393201 & 3,393 & $12 / 01 / 2014$ & 2,894 & 499.4 & UK & Water-level map. \\
\hline $5 \mathrm{~N} / 7 \mathrm{~W}-31 \mathrm{~J}^{1}$ & 342827117384001 & 3,585 & $09 / 24 / 2014$ & 2,947 & 638.5 & 975 & Hydrograph, water-level map. \\
\hline $5 \mathrm{~N} / 7 \mathrm{~W}-31 \mathrm{~J} 4^{1}$ & 342827117383701 & 3,587 & $09 / 24 / 2014$ & 2,956 & 630.7 & 1,120 & Cross section, water-level map. \\
\hline $5 \mathrm{~N} / 8 \mathrm{~W}-7 \mathrm{Q} 1$ & 343150117452101 & 3,093 & $01 / 12 / 2015$ & 2,803 & 290.27 & 400 & Water-level map. \\
\hline $5 \mathrm{~N} / 8 \mathrm{~W}-24 \mathrm{~A} 1^{1}$ & 343045117394301 & 3,296 & $12 / 11 / 2014$ & 2,910 & 386.1 & UK & Cross section, water-level map. \\
\hline $5 \mathrm{~N} / 8 \mathrm{~W}-25 \mathrm{H} 1$ & 342930117393501 & 3,448 & - & - & - & UK & Hydrograph. \\
\hline $5 \mathrm{~N} / 8 \mathrm{~W}-25 \mathrm{~J} 1^{1}$ & 342918117393901 & 3,472 & $09 / 24 / 2014$ & 2,904 & 568 & 1,110 & Hydrograph, water-level map. \\
\hline $5 \mathrm{~N} / 8 \mathrm{~W}-26 \mathrm{~B} 2$ & 342950117405601 & 3,411 & $10 / 16 / 2014$ & 2,910 & 500.67 & 1,000 & Cross section, water-level map. \\
\hline $5 \mathrm{~N} / 8 \mathrm{~W}-26 \mathrm{~B} 3$ & 342953117410201 & 3,404 & $10 / 16 / 2014$ & 2,913 & 491.28 & 980 & Hydrograph, water-level map. \\
\hline $5 \mathrm{~N} / 9 \mathrm{~W}-1 \mathrm{G} 1$ & 343302117461601 & 2,953 & 09/08/2014 & 2,786 & 167.2 & UK & Water-level map. \\
\hline $5 \mathrm{~N} / 9 \mathrm{~W}-4 \mathrm{C} 1$ & 343328117495001 & 2,863 & 03/02/2015 & 2,747 & 115.4 & UK & Hydrograph, water-level map. \\
\hline $5 \mathrm{~N} / 9 \mathrm{~W}-4 \mathrm{~F} 1$ & 343307117493501 & 2,885 & - & - & - & ${ }^{4} 197$ & Hydrograph. \\
\hline $5 \mathrm{~N} / 9 \mathrm{~W}-5 \mathrm{R} 2$ & 343242117500601 & 2,911 & 03/02/2015 & 2,798 & 113.18 & 137.3 & Hydrograph, cross section, water-level map. \\
\hline
\end{tabular}


Table 4. Selected wells with groundwater-level data, 1990-2015, near Piñon Hills, California._Continued

[Depths are below land surface. Altitudes are referenced to the North American Vertical Datum of 1988. See figure 10 for groundwater-level map; see figure 11 for hydrographs; see figure 12 for cross sections. Abbreviations: ft, foot; mm/dd/yyyy, month/day/year; UK, unknown; —, not applicable]

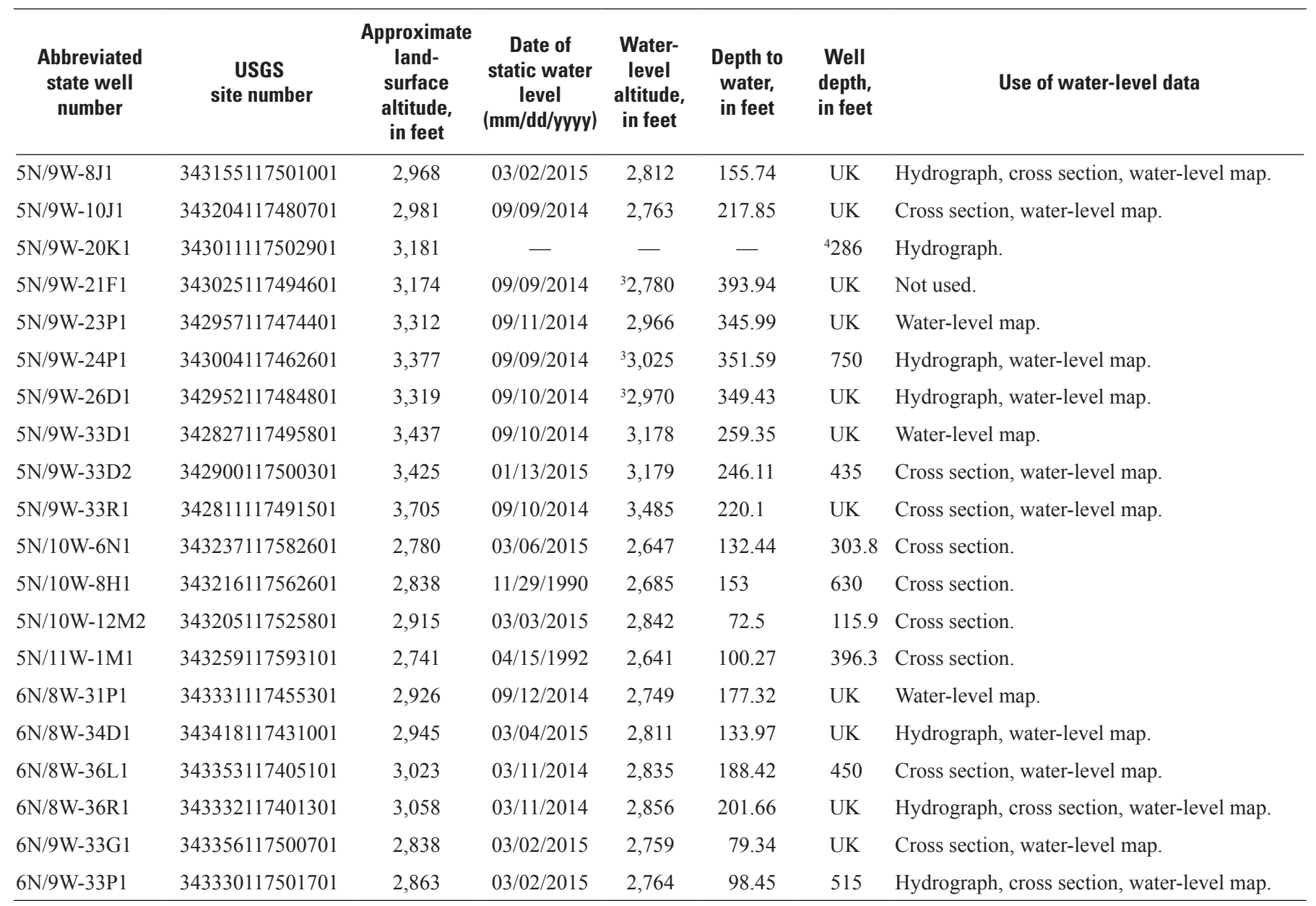

${ }^{1}$ Data reported by Phelan-Piñon Hills Community Services District.

${ }^{2}$ Oil observed in well.

${ }^{3}$ Well recently pumped prior to measurement.

${ }^{4}$ Hole depth. 


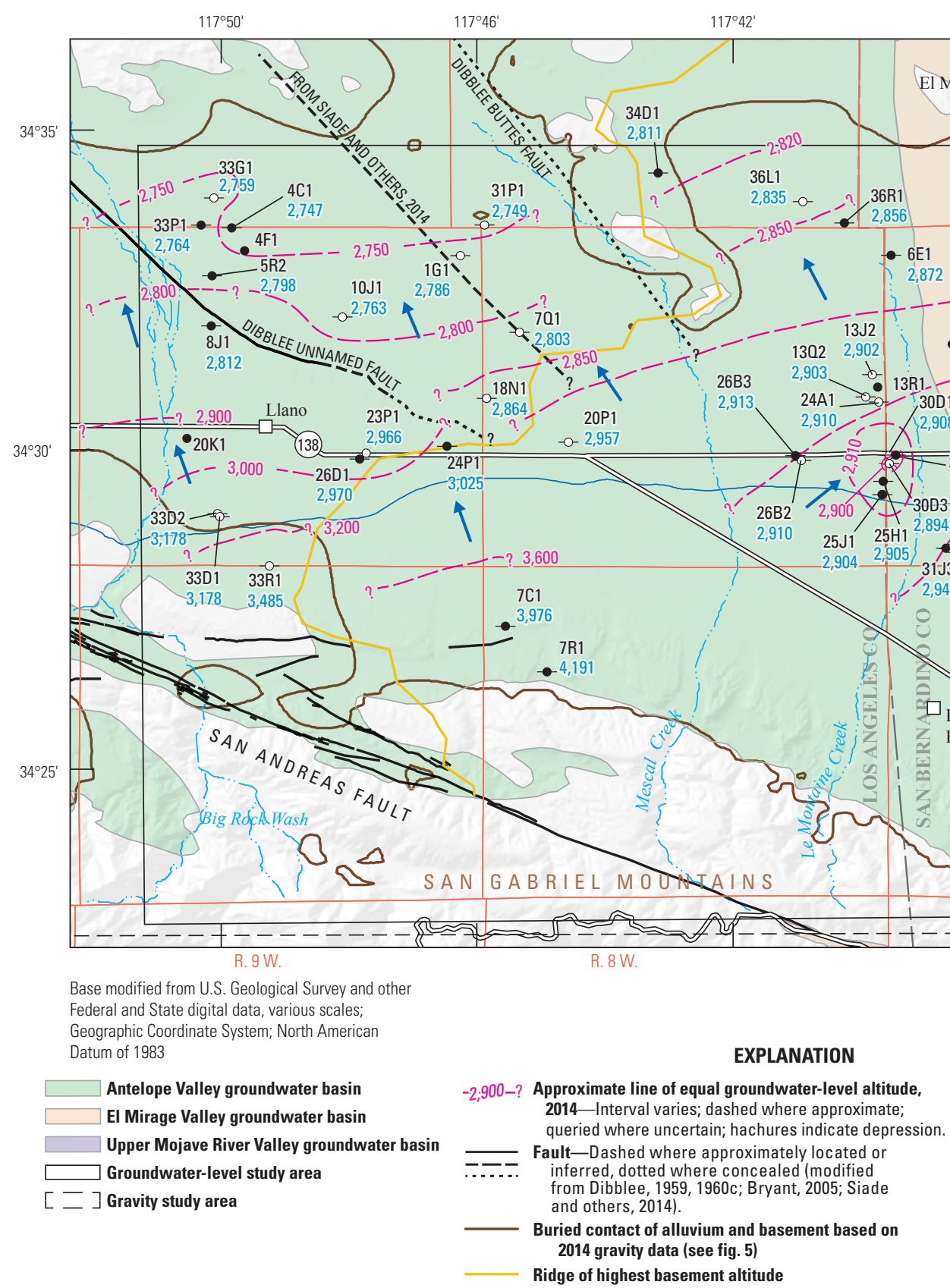

$117^{\circ} 38^{\circ}$

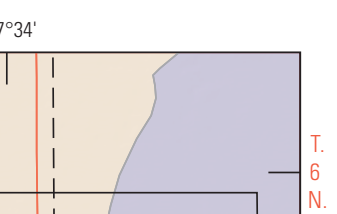


$\boldsymbol{A}$

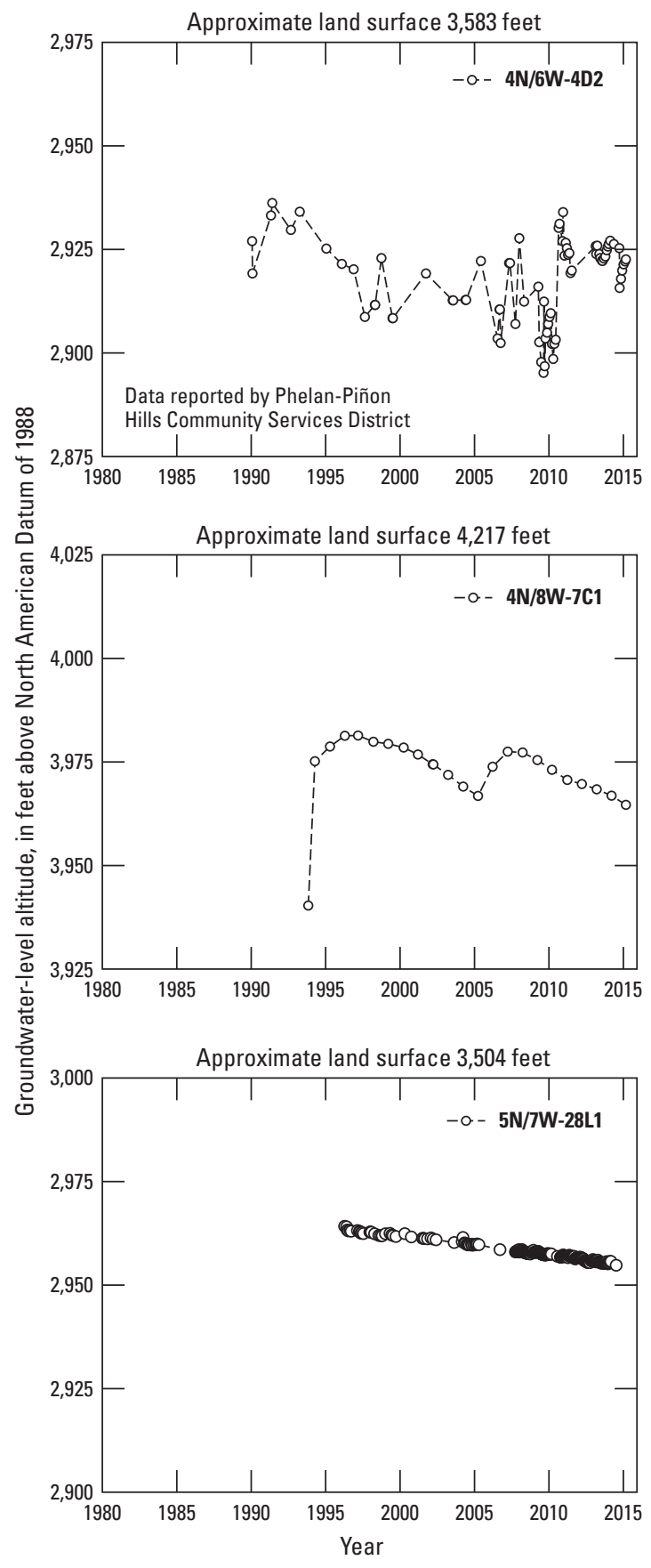

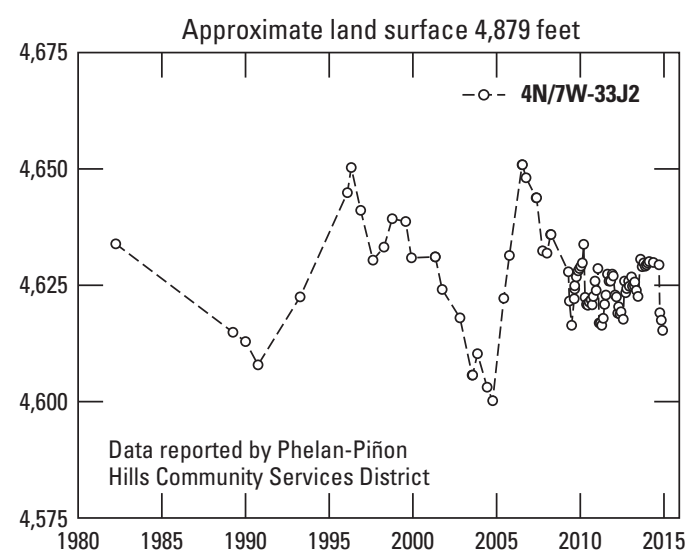

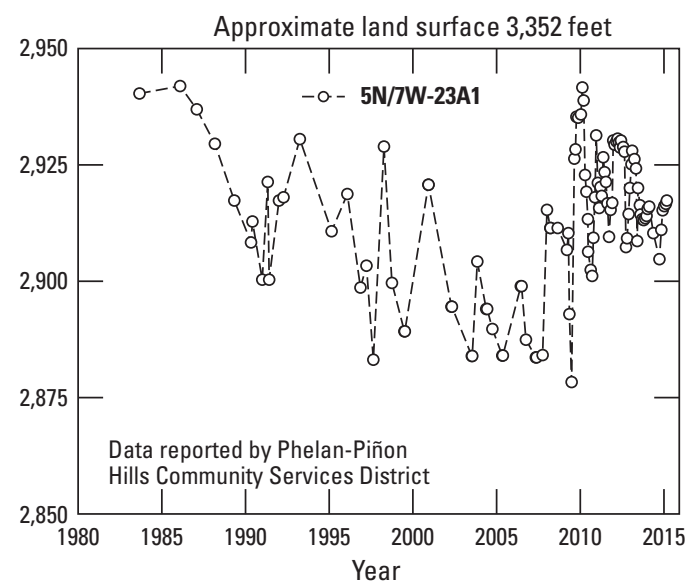

Figure 11. Groundwater level in wells. A, 1980-2015, and B, 1950-2015 and 1915-2015, near Piñon Hills, California. (Location of wells shown in figure 10.) 
A
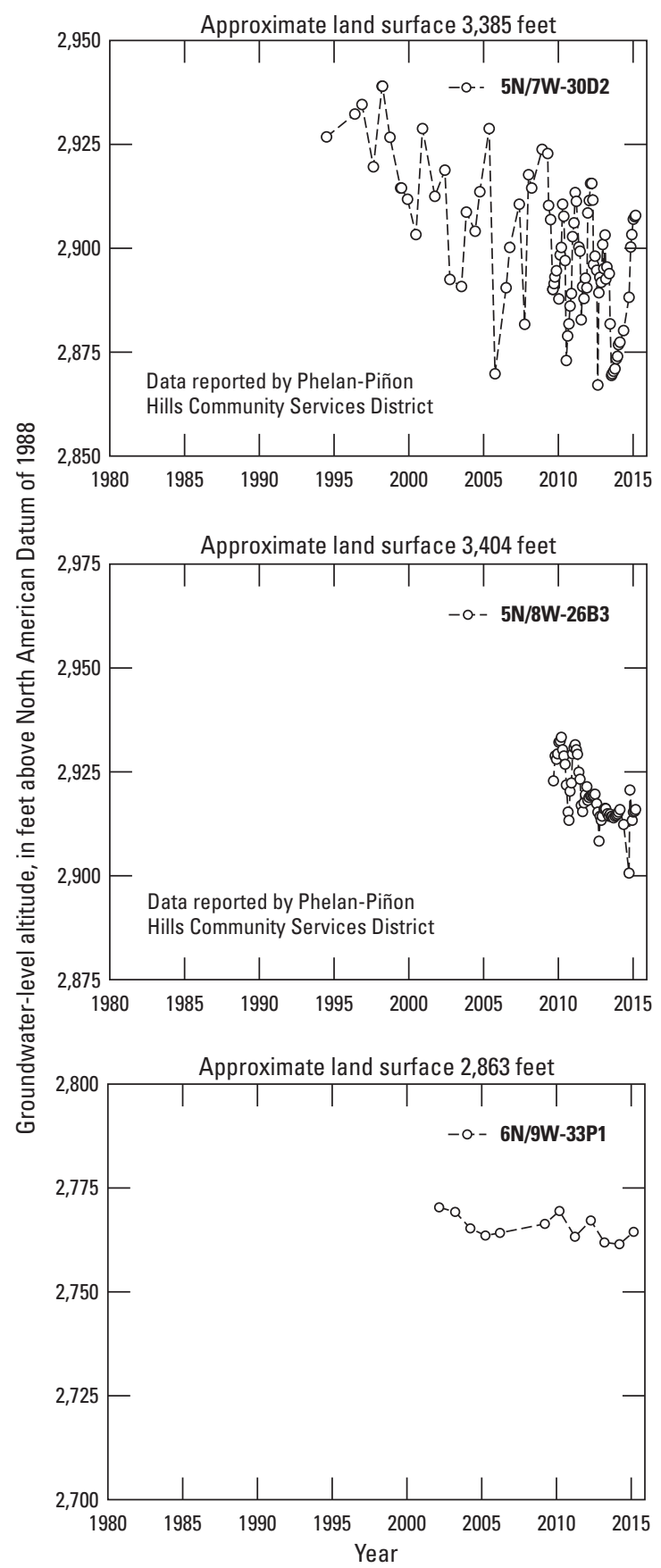
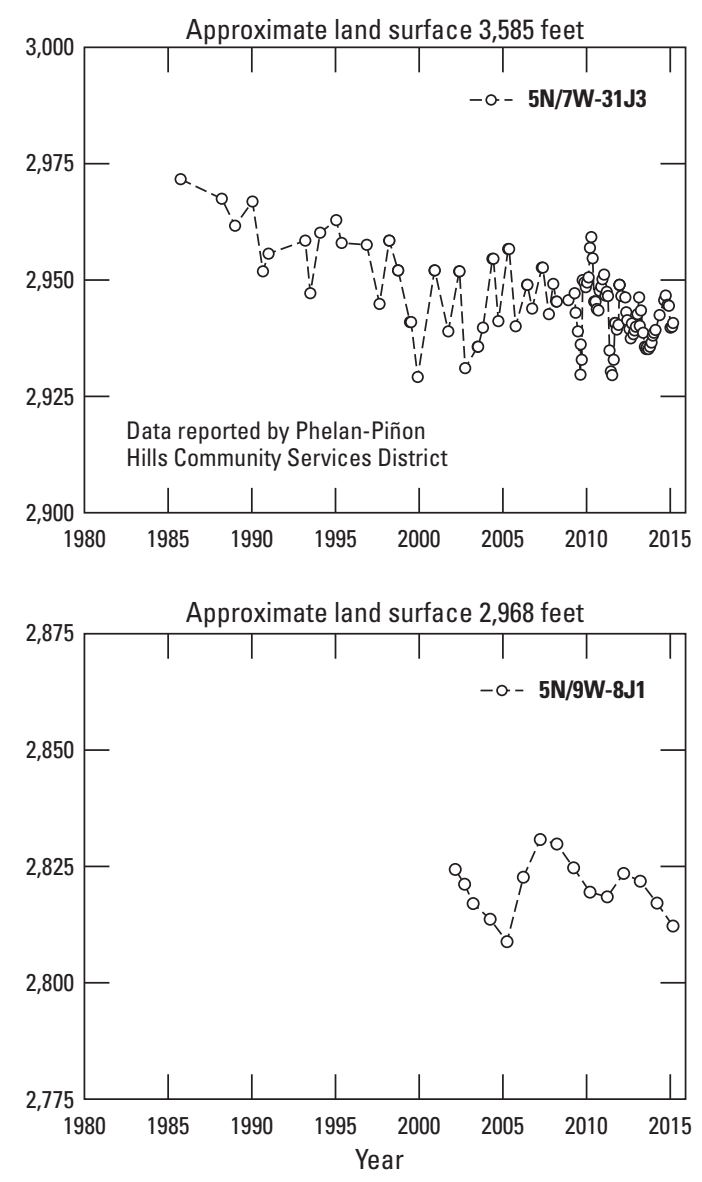

Figure 11. - Continued 
$B$

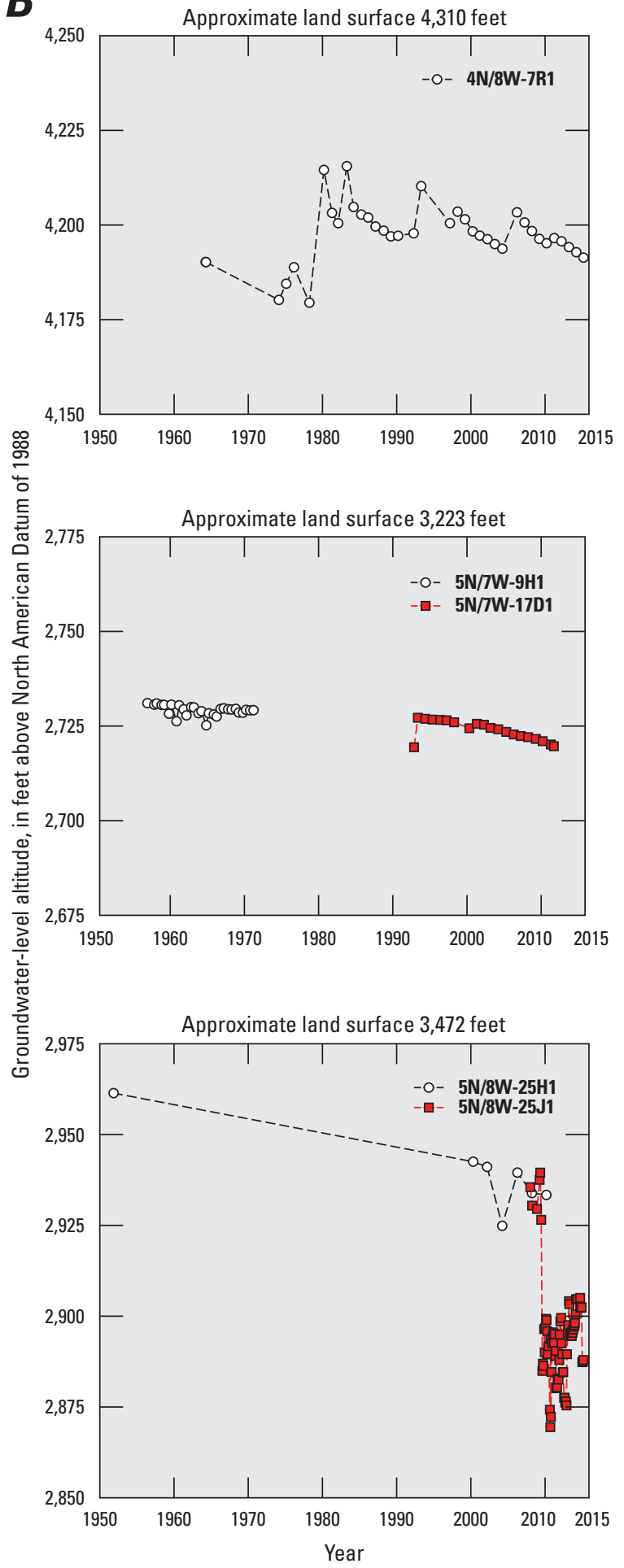

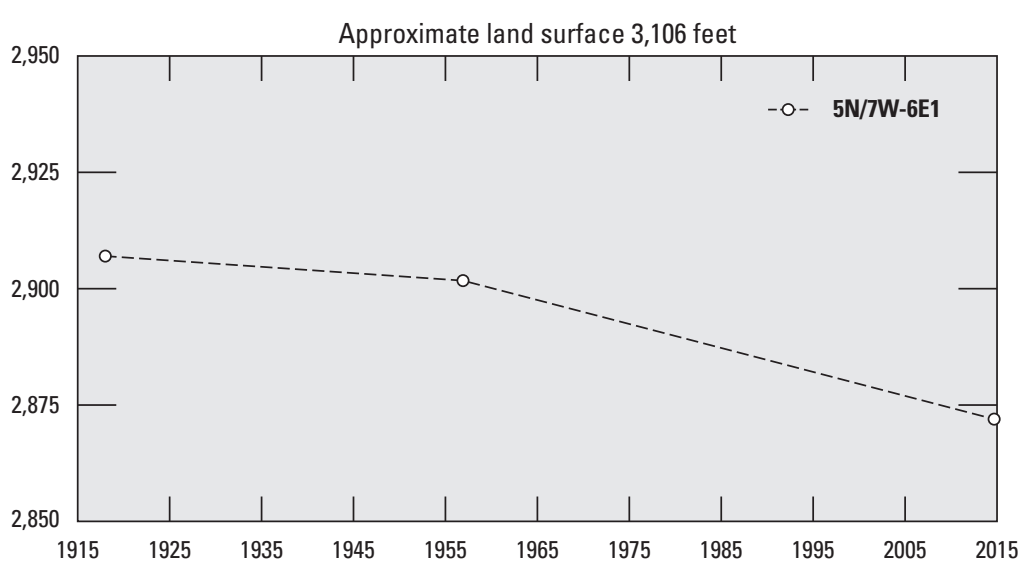
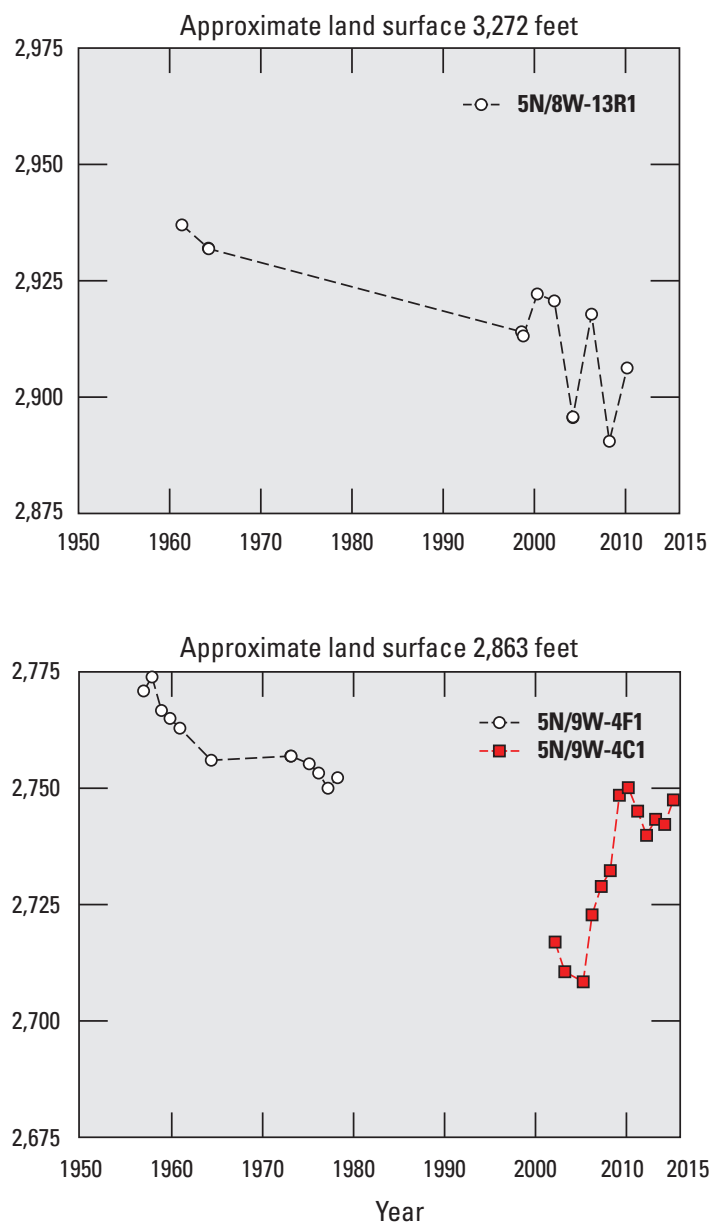

Figure 11. - Continued 
B

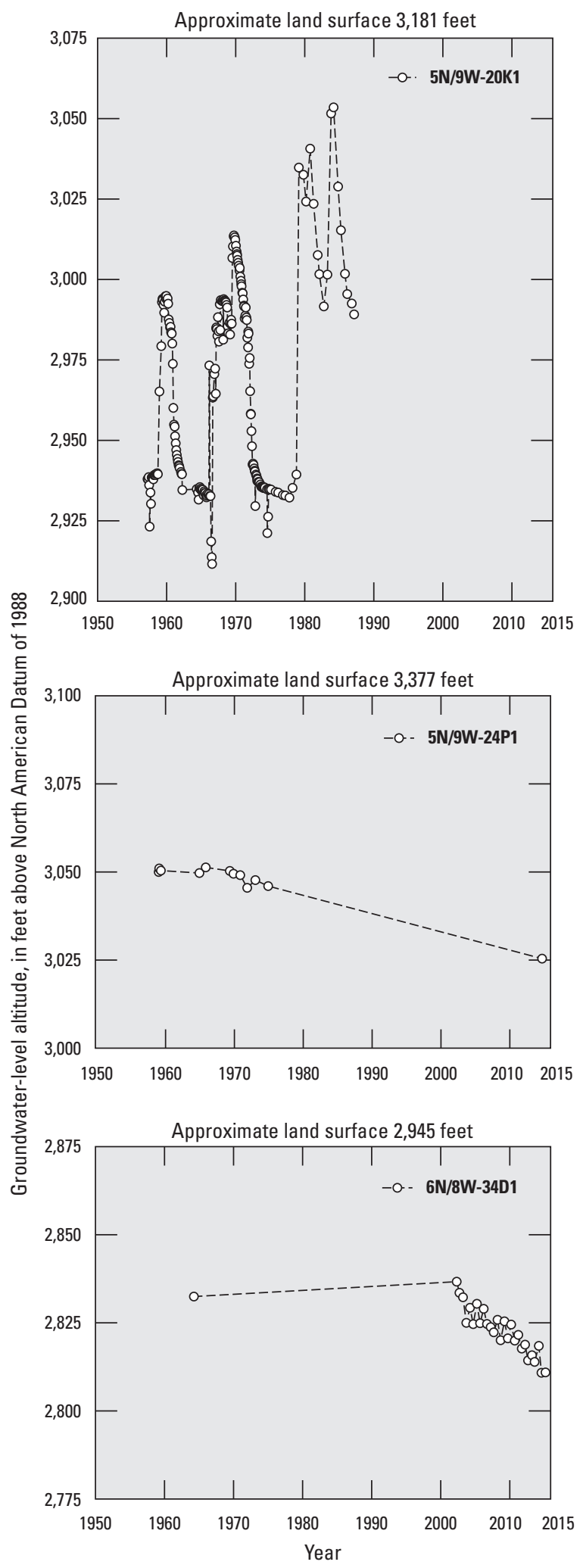

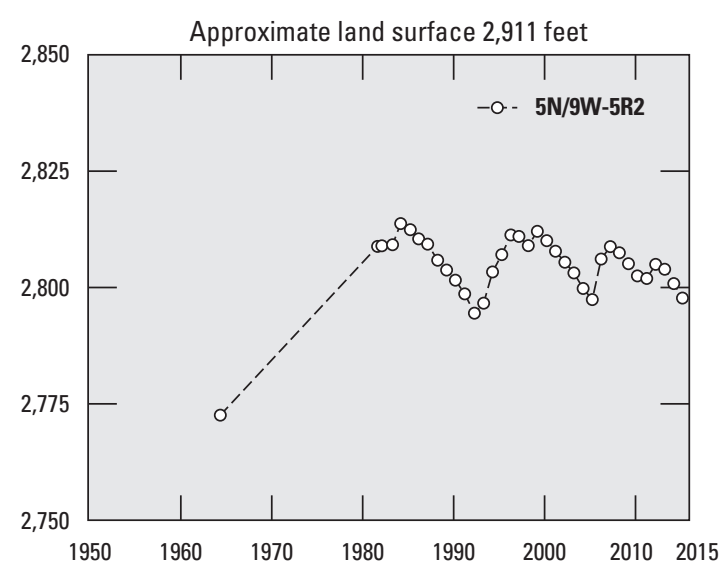
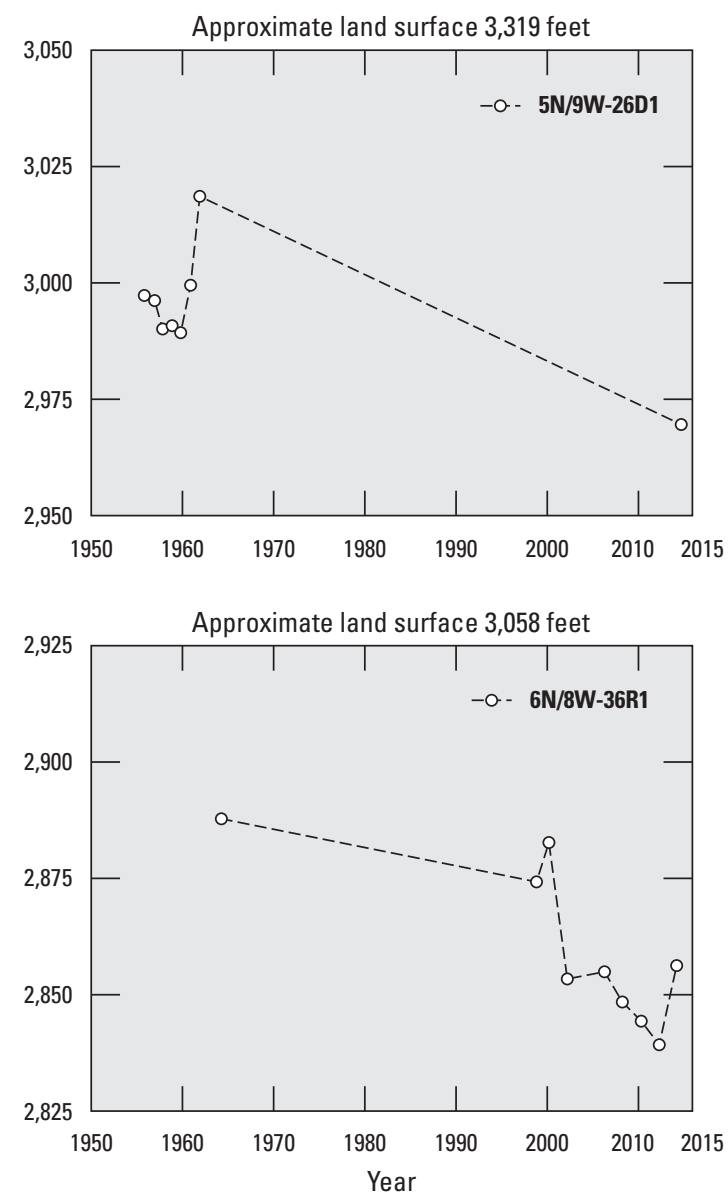

Figure 11. - Continued 
Most of the hydrographs show groundwater-level fluctuations caused by seasonal variation in pumping from the well, or pumping from nearby wells, and also show an overall trend of groundwater-level decline. Groundwater-level data from well 5N/8W-13R1, near and downgradient from most of the pumping (fig. 9, 11B), show a decline of about $30 \mathrm{ft}$ between 1961 and 2010. Data from well 5N/8W-26B3, about $1.5 \mathrm{mi}$ west of the Los Angeles-San Bernardino County line in an area with high pumping rates, show a decline of about $18 \mathrm{ft}$ between 2010 and 2015 (figs. 10, 11A). Data from observation well 5N/7W-28L1, in Sheep Creek Wash about 2.3 mi east of the Los Angeles-San Bernardino County line in an area with minor groundwater-level fluctuations from pumping wells, show a steady decline in groundwater levels of more than 0.5 foot per year (ft/yr), or about $9 \mathrm{ft}$ between 1996 and 2014 (fig. 11A).

Wells near the mountain front $(4 \mathrm{~N} / 8 \mathrm{~W}-7 \mathrm{C} 1$ and 4N/8W-7R1) and along Big Rock Wash (5N/9W-5R2, $5 \mathrm{~N} / 9 \mathrm{~W}-8 \mathrm{~J} 1$, and $6 \mathrm{~N} / 9 \mathrm{~W}-33 \mathrm{P} 1$; figs. 10,11 ) show smaller overall declines in groundwater levels over time, likely in response to occasional recharge from runoff from the San Gabriel Mountains. The increases in groundwater levels in these wells correlate to periods of runoff estimated from the regional-scale Basin Characterization Model (BCM) for the Antelope Valley (Siade and others, 2014). Shallow wells along Big Rock Wash where the alluvium is thin $(5 \mathrm{~N} / 9 \mathrm{~W}-4 \mathrm{C} 1$ and $5 \mathrm{~N} / 9 \mathrm{~W}-20 \mathrm{~K} 1$; figs. $10,11 B$ ) show large fluctuations in groundwater levels in response to pumping and natural recharge. Groundwater likely is affected by the presence of shallow basement rocks and correspondingly thin alluvium in the area of Big Rock Wash (fig. 5), resulting in little or no saturated thickness.

\section{Accessing Groundwater-Level Data}

The groundwater-level data presented in this report can be accessed through the USGS National Water Information System Web service (NWISWeb) at http://waterdata.usgs. gov/nwis/ and can be accessed by interactive map with NWIS Mapper at http://maps.waterdata.usgs.gov/. The NWISWeb serves as an interface to a database of site information and real-time groundwater, surface-water, and water-quality data collected from locations throughout the United States and elsewhere. Data can be retrieved by State, category, and geographic area and can be selectively refined by specific location or parameter field. The NWISWeb can output groundwaterlevel and water-quality graphs, site maps, and data tables (in HTML and ASCII format).

Available groundwater-level data for the study area can be accessed from the NWISWeb http://nwis.waterdata.usgs. gov/ca/nwis/gwlevels.

\section{Relation of Groundwater-Basin Thickness to Groundwater Availability}

Combining the results of the gravity and groundwaterlevel surveys shows the relation of the geologic structure of the basement complex with the groundwater system. Three generalized hydrogeologic sections through the study area (fig. 12) were constructed using the depth to basement estimated from the gravity data (fig. 6) and the groundwater-level altitudes measured from wells in 2014-15 (fig. 10; table 4). The west-east section (figs. 6, 12A) shows that the saturated alluvium is thinner to the west and is thickest to the east and that the crest of the ridge of highest basement altitude is between wells $5 \mathrm{~N} / 8 \mathrm{~W}-18 \mathrm{~N} 1$ and $5 \mathrm{~N} / 8 \mathrm{~W}-20 \mathrm{P} 1$. Based on the groundwater-level data from September 2014, the saturated thickness of the alluvial deposits near well $5 \mathrm{~N} / 8 \mathrm{~W}-18 \mathrm{~N} 1$ was estimated to be about $130 \mathrm{ft}$. Although it is uncertain whether or how much this basement ridge affects the groundwater system, lowering the water table below the elevation of the basement ridge crest could effectively create a boundary to groundwater flow and potentially could cause the area to the west to become hydrologically isolated from the area to the east.

The westernmost south-north section (figs. 6, 12B) shows that the saturated alluvium is thinnest in this part of the study area and that the maximum depth to basement along the section is near well $6 \mathrm{~N} / 9 \mathrm{~W}-33 \mathrm{G} 1$. The altitude of the basement surface is highest at the north and south ends of the basin where it is exposed, and the groundwater level measured in well 5N/9W-33R1 indicates that the water table is probably below or near the basement surface. The drillers' log from well 5N/9W-33D2 encountered "hard rocks" at about 140 feet below land surface (ft bls), confirming that the basement at this location is shallow and could be highly fractured because of the proximity to the San Andreas Fault Zone. Note that the basement surface based on the gravity data inversion is averaged over 1,000 -ft by $1,000-\mathrm{ft}$ grid cells; therefore, basement elevations averaged over a large area may not reflect the exact depth to basement at this well.

The easternmost south-north section (figs. 6, 12C) shows that the saturated alluvial deposits are more than $2,000 \mathrm{ft}$ thick south of well $5 \mathrm{~N} / 7 \mathrm{~W}-31 \mathrm{~J} 4$ but gradually thin to the north to a thickness of about $500 \mathrm{ft}$; basement outcrops are evidence of the thinning of the alluvial deposits at the north edge of the study area. Because the locations of the sections were determined by the limited availability of wells with groundwaterlevel data, it is uncertain how the basement highs indicated by the gravity data southeast of well 5N/7W-31J4, and any potential changes in the water-table altitude, could affect groundwater flow near this area (figs. 6, 12C) . 

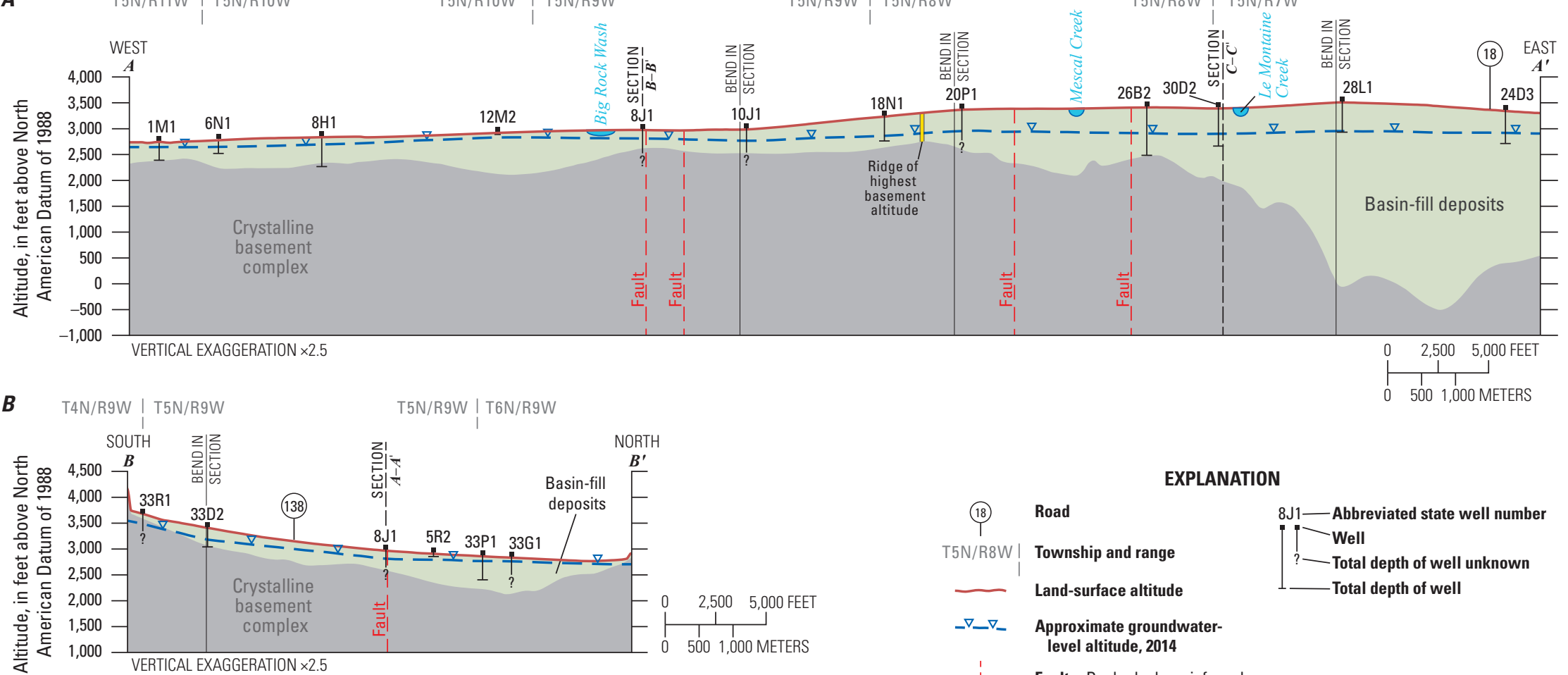

EXPLANATION
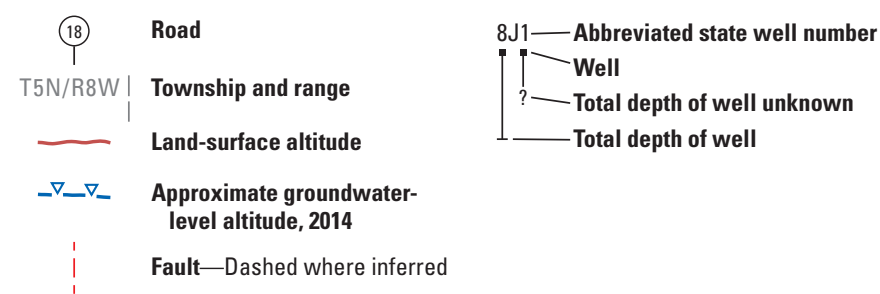

Figure 12. Generalized hydrogeologic cross sections showing groundwater-level altitude in 2014-15 and estimated altitude of the basement complex from the modeled gravity data: $A$, west to east $\left(A-A^{\prime}\right)$; $B$, south to north $\left(B-B^{\prime}\right)$; and $C$, south to north $\left(C-C^{\prime}\right)$, near Piñon Hills, California. (Lines of section shown in figure 6 .) 
To help further visualize the relation between the topography of the basement surface and the groundwater system in the study area, two 3-D animations of the altitude of the basement and groundwater table were prepared. The animations allow the reader to see this relation from many angles and perspectives. The vertical features are greatly exaggerated in the animation to better illustrate the basin structure and the saturated alluvial thickness. To construct the animations, the altitude of the top of the basement was calculated by subtracting the modeled thickness of the basin-fill deposits from the average land-surface altitude of each gravity grid cell. The surface of the top of the water table was created by subtracting the depth to water measured at each well in 2014-15 from the respective land-surface datum.

The first animation is a flyover that initially shows land surface and other landmarks to orient the viewer and then shows the altitude of the top of the basement (grey shaded relief) and the saturated thickness of the groundwater system (fig. 13). The altitude of the top of the basement rocks is shown in graduated color (purple) and by contours of $250-\mathrm{ft}$ intervals. The blue dots indicate where the water table was encountered in a well during 2014-15, and the vertical red lines indicate the saturated thickness of the alluvium based on the gravity calculations and groundwater-level data collected for this study.
The second animation demonstrates the potential effects of dewatering the saturated alluvial deposits and indicates areas of possible groundwater divides if the altitude of the groundwater table declines below the ridge of the highest basement altitude (fig. 14). As discussed earlier, the saturated alluvium is thinnest in the middle of the study area (fig. 12); the ridge of the highest basement altitude is indicated by a yellow line on figures $6,10,12$, and 14. After showing landmarks to orient the viewer looking towards the southwest, the animation shows the water-table altitude estimated from the depth-to-water measurements from 2014-15, then shows a simulated incremental decline of the water table (fig. 14). The animation was prepared with the following assumptions: (1) the groundwater-table decline was simulated as simple gravity drainage, incrementally declining at a rate of $16.4 \mathrm{ft}$ (5 meters) per frame; (2) the gradient of the 2014-15 estimated watertable surface was maintained; (3) the incremental drawdown of the water-table surface was applied equally everywhere; and (4) no recharge or discharge occurred. In addition, the animation does not predict any timeframe for dewatering nor address hydrologic factors that could affect groundwater flow such as vertical and horizontal conductivity or heterogeneity within the alluvium, the presence or absence of vertical structures (such as faults), or alterations in groundwater flow from pumping.

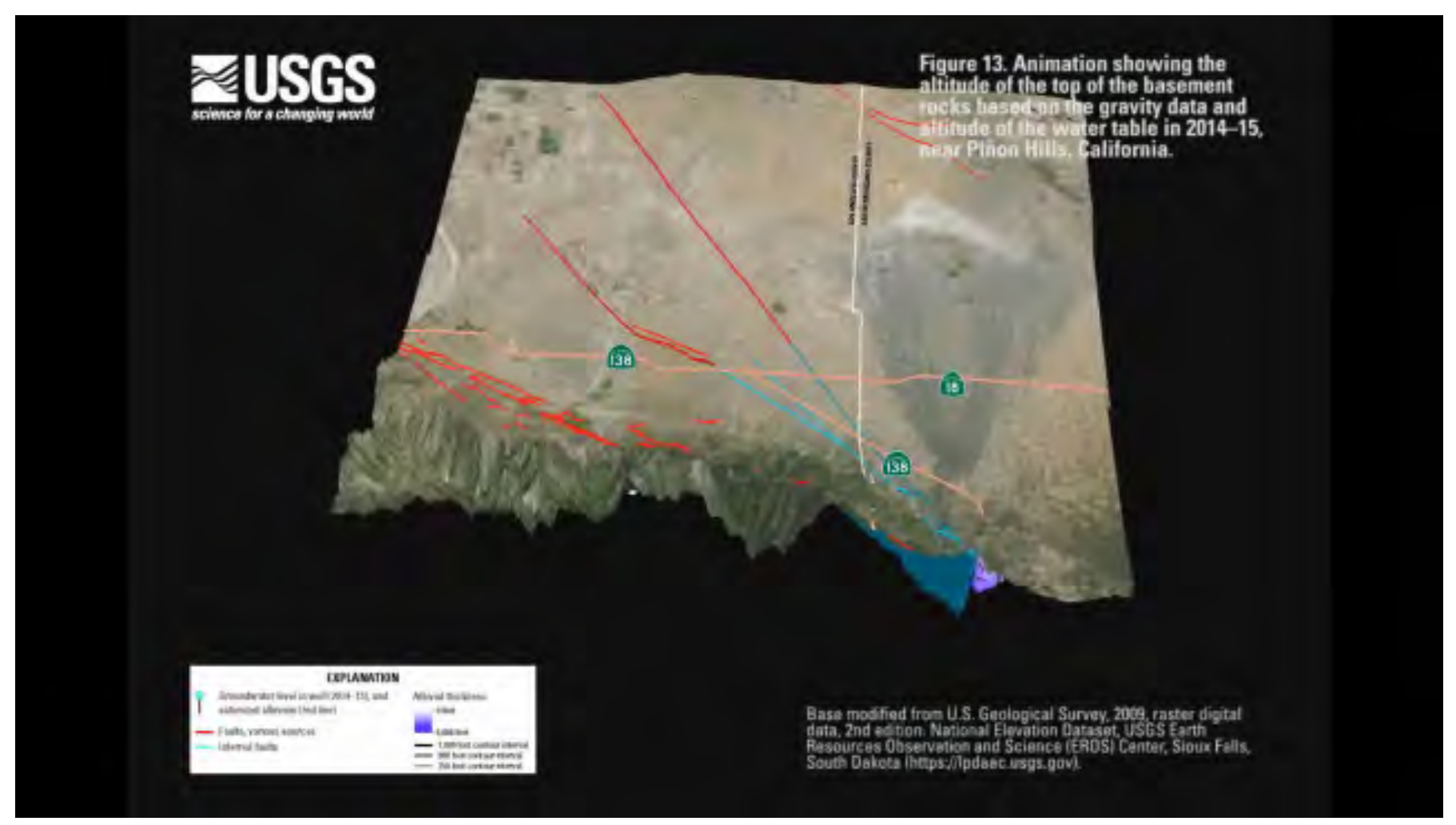

Figure 13. Animation showing the altitude of the top of the basement rocks based on the gravity data and altitude of the water table in 2014-15, near Piñon Hills, California. Visit https://doi.org/10.3133/sir20175065 for animation file. 


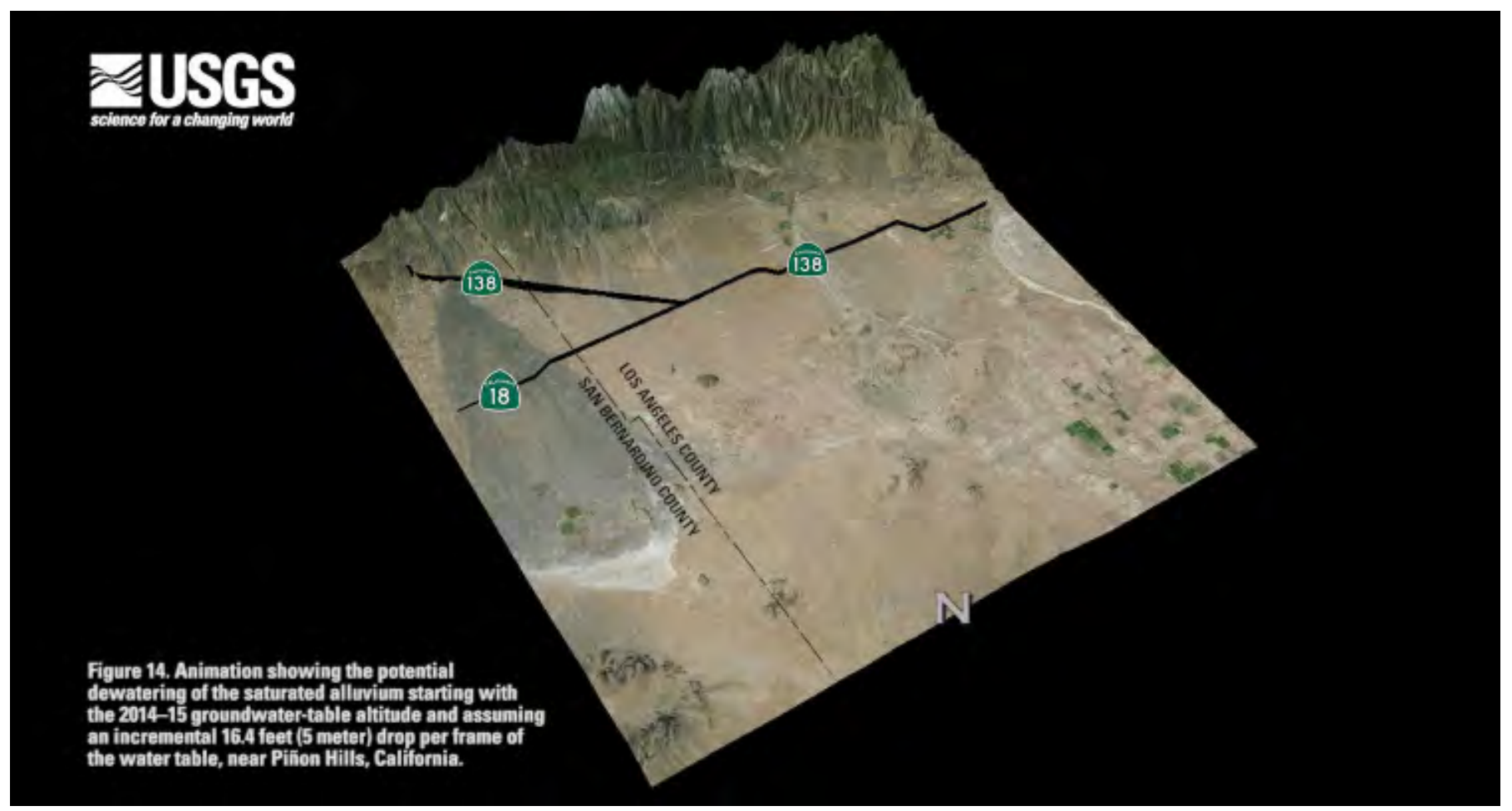

Figure 14. Animation showing the potential dewatering of the saturated alluvium starting with the 2014-15 water-table altitude and assuming an incremental 16.4 feet (5 meter) drop per frame of the water table, near Piñon Hills, California. Visit https://doi.org/10.3133/ sir20175065 for animation file.

\section{Limitations and Considerations for Future Studies}

The work done for this study approximates the basement morphology, thickness of the basin fill, and suggests possible structures, such as basement ridges and extension of known faults, that could affect groundwater flow. The results cannot be used on their own to estimate quantities of groundwater flow between areas, but this information can help guide future investigations. Limitations of the interpretation of the gravity data include the averaging of the shape of the basin-fill deposits, which is more pronounced where those deposits are the thickest. Also, the calculations of depth to basement assumed that there were no lateral variations in density of the basinfill deposits, which may not be representative if the deposits coarsen toward the mountain front. Because of the few existing wells that have encountered or penetrated the basement, the calculated depth to basement from the gravity data may be better refined by the results of further geophysical and exploratory methods. Surface geophysical methods such as seismic, resistivity, or time-domain electromagnetic (TEM) could help to constrain the altitude of the basement surface and provide additional details of the stratigraphy, grain size, and structure (including the location of faults) within the basin-fill deposits.
Data from exploratory drilling and the installation and sampling of multi-depth monitoring wells also can be used to help (1) refine the subsurface geology and location of potential barriers to groundwater flow; (2) determine vertical and lateral hydraulic gradients; (3) determine depth-dependent groundwater quality and age; and (4) suggest potential sources of groundwater recharge to the area, such as water derived from higher-altitude drainages like Sheep Creek Wash and small streams draining lower-altitude areas of the San Gabriel Mountains. The basin-fill deposits thickness map (fig. 5) prepared for this study could be used to help identify the location of potential boreholes to investigate the water-bearing properties of the groundwater basin.

Another consideration could be to develop a local-scale model of the study area or to link together the existing, but separate, groundwater-flow models of the Antelope Valley groundwater basin (Siade and others, 2014) and Mojave Basin Area (Stamos and others, 2001) into a single calibrated groundwater-flow model. Such a model could be used to better characterize or help quantify the uncertainty of the boundary characteristics and flow between the basins. This model could help quantify the flow and its uncertainty across the jurisdictional boundary under a variety of future hydrologic conditions and groundwater-management scenarios. 


\section{Summary}

The increasing demands on limited groundwater resources for water supply in desert areas and recent legislation enacted in California have resulted in the need to better understand groundwater sources, supply, and sustainability. The Antelope Valley, El Mirage Valley, and Upper Mojave River Valley groundwater basins in the western part of the Mojave Desert have been investigated previously in separate studies and have been adjudicated in separate judgments, but no widely documented hydrogeologic study has been completed to define the basin geometry or to characterize the groundwater system near the boundary between these adjacent groundwater basins. In an effort to assess if the boundary between the Antelope Valley and El Mirage Valley groundwater basins could be better defined or determined, the U.S. Geological Survey began a cooperative study in 2014 with the Mojave Water Agency to evaluate the hydrogeology of the area. Geophysical, geological, and hydrological data and interpretations are sparse in the study area, which covers about 650 square miles $\left(\mathrm{mi}^{2}\right)$ and straddles the southern parts of the Los Angeles and San Bernardino Counties. This study included completion of a gravity survey, the compilation of existing information on wells in the study area, a field reconnaissance to search for new existing wells, and the gathering of groundwater-level data from wells. The gravity-derived model results enabled estimates of the thickness of the basinfill deposits (or depth to the top of basement in the area) and helped determine the location of basement structures that could influence groundwater flow now or in the future. Gravity results were combined with the groundwater-level data to determine the thickness of the saturated alluvial deposits and highlight areas where groundwater flow could be controlled or influenced by the topography of the basement.

The area of the Mojave Desert comprising the Antelope Valley, El Mirage Valley, and Upper Mojave River Valley groundwater basins contains large, mostly alluvial-filled structural depressions downfaulted between the Garlock and San Andreas Fault Zones. The underlying basement complex, not considered water bearing, forms the base and margins of the groundwater basins and crops out in highlands within the study area. A regional gravity survey by previous investigators indicated the presence of faults within the basin-fill deposits and many west-northwest striking faults that are parallel to the main strands of the San Andreas Fault Zone. Three faults have been mapped in the alluvial deposits, but their southeastern extent and relation to the San Andreas Fault are unknown; also unknown is whether they affect groundwater flow.

A previously published regional-scale gravity study of the western Mojave Desert covered an area of about 7,500 $\mathrm{mi}^{2}$ with coarsely spaced data points. Preliminary analysis of the regional gravity data suggested a subsurface northeasttrending basement ridge and saddle approximately 3.5 miles west of the boundary between the Antelope Valley and El Mirage Valley groundwater basins. To augment the regional gravity study and refine the modeled basement surface, a more detailed gravity survey covering an area of about $200 \mathrm{mi}^{2}$ was done as part of this study. Drillers' logs from 57 boreholes were used to constrain the gravity-interpreted thickness of the basin-fill deposits. The interpretation of the gravity data resulted in an estimated thickness of the basin-fill deposits, or depth to basement complex, which ranged from 0 feet $(\mathrm{ft})$ in the San Gabriel Mountains along the San Andreas Fault Zone to about 5,775 $\mathrm{ft}$ in the northwestern and southwestern parts of the study area. The gravity survey results also confirmed that two basement depressions in the northwestern and southeastern parts of the study area separated by a ridge of highest basement altitude trending generally north to south. The mapped surface expressions of the faults in the study area generally are parallel to the trend of the basement gravity field contours and coincide with the alignment of a basement ridge to the southwest of the Three Sister Buttes. Based on the basement gravity field and estimates of basement thickness, it may be reasonable to extend the Dibblee Buttes Fault to the southeast toward the San Andreas Fault Zone.

A comprehensive field-reconnaissance survey was done in September 2014 to describe the location of existing wells, to collect groundwater-level data and determine the depth to the water table, and to possibly determine the direction of groundwater flow; additional water-level data collected from March 2015 through March 2015 were used to augment the field data. The direction of groundwater flow in the eastern part of the study area is south to north and subparallel to the shared boundary of the Antelope Valley and El Mirage Valley groundwater basins; in the western part of the study area, groundwater flow is northwesterly. This suggests that some groundwater flow could occur from the El Mirage Valley groundwater basin to the Antelope Valley groundwater basin, but insufficient groundwater-level data were available to confirm this. Future patterns of regional groundwater flow likely will be influenced by changes in the number and distribution of pumping wells, the amount of pumpage withdrawn from the aquifer system, and the thickness of the saturated alluvium. The groundwater-level data from wells in the northwestern part of the study area suggested that the faults could have some effect on groundwater flow and that groundwater flows out of the study area to the northwest.

Combining the results of the gravity and groundwaterlevel surveys shows the relation of the geologic structure in the study area with the groundwater system. The gravity and groundwater-level data showed that the saturated alluvium was thickest in the northwestern and southeastern parts of the study area, was thinner to the west and middle of the study area, and that the crest of a ridge of the highest basement altitude was east of well $5 \mathrm{~N} / 8 \mathrm{~W}-18 \mathrm{~N} 1$, where the alluvium was estimated to be about $130 \mathrm{ft}$ thick. Although it was uncertain whether the ridge of the highest basement altitude affects the groundwater system, a potential barrier to groundwater flow could be created if the water table fell below the altitude of the basement ridge, effectively causing the area to the west of the basement ridge to become hydraulically isolated from the area to the east. To help further visualize the relation between topography of the basement surface and the groundwater system in the area, two three-dimensional (3-D) animations showing the characteristics of the basement and groundwater table were prepared. 


\section{References Cited}

Bloyd, R.M., 1967, Water resources of the Antelope ValleyEast Kern Water Agency area, California: U.S. Geological Survey Open-File Report 67-21, 73 p.

Bryant, W.A. (compiler), 2005, Digital database of Quaternary and younger faults from the fault activity map of California, version 2.0: California Geological Survey web page, accessed September 9, 2015, at http://www.conservation. ca.gov/cgs/information/publications/Pages/quaternaryfaults_ver2.aspx.

California Department of Conservation, 2016, Division of Oil, Gas, and Geothermal Resources Well Finder: DOGGR Well Finder web page, accessed September 22, 2016, at http:// www.conservation.ca.gov/dog/Pages/WellFinder.aspx.

California Department of Water Resources, 1966, Data on water wells in the eastern part of the Antelope Valley area, Los Angeles County, California: California Department of Water Resources, Bulletin 9-12, 448 p.

California Department of Water Resources, 1967, Mojave River ground water basins investigation: California Department of Water Resources, Bulletin 84, 151 p.

California Department of Water Resources, 2003, California's groundwater: California Department of Water Resources, Bulletin 118, Update 2003, accessed March 30, 2016, at http://www.water.ca.gov/groundwater/bulletin118/ report2003.cfm.

California Department of Water Resources, 2015a, California State Water Project Overview: California Department of Water Resources, accessed November 30, 2015, at http://www.water.ca.gov/swp/.

California Department of Water Resources, 2015b, California Sustainable Groundwater Management Act: California Department of Water Resources, accessed December 29, 2015, at http://www.water.ca.gov/cagroundwater/moresgma.cfm.

California Department of Water Resources, 2016, List of adjudicated basins and subbasins: California Department of Water Resources, accessed February 1, 2016, at http:// www.water.ca.gov/groundwater/docs/List $\% 20$ of $\% 20$ adjudicated $\% 20$ basins $\% 20$ and $\% 20$ subbasins_01012014.pdf.

California Irrigation Management Information System, 2015, California ETo zones map: California Department of Water Resources, accessed July 22, 2015, at http://wwwcimis. water.ca.gov/App_Themes/images/etozonemap.jpg.

Carlson, C.S., Leighton, D.A., Phillips, S.P., and Metzger, L.F., 1998, Regional water table (1996) and water-table changes in the Antelope Valley ground-water basin, California: U.S. Geological Survey Water-Resources Investigations Report 98-4022, 2 plates.
Christensen, A.H., 2005, Generalized groundwater-level contours, September-October 2000 and March-April 2001, and long-term groundwater-level changes, at the U.S. Air Force Plant 42 and vicinity, Palmdale, California: U.S. Geological Survey Scientific Investigations Report 2005-5074, 130 p.

Dibblee, T.W., Jr., 1959, Geologic map of the quadrangle, California: Mineral Investigations Field Studies Map MF-222, accessed at https://ngmdb.usgs.gov/Prodesc/proddesc_2641. htm, scale 1:62:500.

Dibblee, T.W., Jr., 1960a, Geologic map of the Lancaster quadrangle, Los Angeles County, California: U.S. Geological Survey Miscellaneous Field Studies Map 76, scale 1:62:500.

Dibblee, T.W., Jr., 1960b, Geologic map of the Shadow Mountains quadrangle, Los Angeles and San Bernardino Counties, California: U.S. Geological Survey Miscellaneous Field Studies Map 76, scale 1:62:500.

Dibblee, T.W., Jr., 1960c, Preliminary geologic map of the Shadow Mountains quadrangle, Los Angeles and San Bernardino Counties, California: Mineral Investigations Field Studies Map MF-227, accessed at https://ngmdb.usgs.gov/ Prodesc/proddesc_94.htm, scale 1:62:500.

Dibblee, T.W., Jr., 1963, Geology of the Willow Springs and Rosamond quadrangles, California: U.S. Geological Survey Bulletin 1089-C, p. 73-139. [Also available at https://pubs. er.usgs.gov/publication/b1089C.]

Dibblee, T.W., Jr., 1967, Areal geology of the Western Mojave Desert, California: U.S. Geological Survey Professional Paper 552, $161 \mathrm{p}$.

Dibblee, T.W., Jr., 2002a, Geologic Map of the Mescal Creek Quadrangle, Los Angeles and San Bernardino Counties, California: Dibblee Geological Foundation Map DF81, scale $1: 24,000$.

Dibblee, T. W. Jr., 2002b, Geologic Map of the Juniper Hills Quadrangle, Los Angeles County, California: Dibblee Geological Foundation Map DF79, scale 1:62,500.

Dibblee, T.W., Jr., 2002c, Geologic Map of the Valyermo quadrangle, Los Angeles County, California: Dibblee Geological Foundation Map DF80, scale 1:24,000.

Dibblee, T.W., Jr., 2008, Geologic Map of the Shadow Mountains and Victorville quadrangles, Los Angeles and San Bernardino Counties, California: Dibblee Geological Foundation Map DF387, scale 1:62,500.

Dokka, R.K, and Travis, C.J., 1990, Late Cenozoic strike-slip faulting in the Mojave Desert, California: Tectonics, v. 9, no. 2, p. 311-340.

Durbin, T.J., 1978, Calibration of a mathematical model of the Antelope Valley ground-water basin, California: U.S. Geological Survey Water-Supply Paper 2046, 51 p., 13 plates. 
Dutcher, L.C., and Worts, G.F., 1963, Geology, hydrology, and water supply of Edwards Air Force Base, Kern County, California: U.S. Geological Survey Open-File Report 63-146, $225 \mathrm{p}$.

Faunt, C.C., 1997, Effect of faulting on ground-water movement in the Death Valley Region, Nevada and California: U.S. Geological Survey Water-Resources Investigations Report 95-4132, 42 p.

Flint, L.E., and Martin, Peter, eds., with contributions by Brandt, Justin, Christensen, A.H., Flint, A.L., Flint, L.E., Hevesi, J.A., Jachens, Robert, Kulongoski, J.T., Martin, Peter, and Sneed, Michelle, 2012, Geohydrology of Big Bear Valley, California; Phase 1-Geologic framework, recharge, and preliminary assessment of the source and age of groundwater: U.S. Geological Survey Scientific Investigations Report 2012-5100, $112 \mathrm{p}$.

Fuis, G.S., Ryberg, T., Godfrey, N.J., Okaya, D.A., and Murphy, J.M., 2001, Crustal structure and tectonics from the Los Angeles basin to the Mojave Desert, southern California: Geology, v. 29, no. 1, p. 15-18.

Hardt, W.F., 1971, Hydrologic analysis of Mojave River basin, California, using electric analog model: U.S. Geological Survey Open-File Report 72-157, 84 p.

Hewett, D.F., 1954, General geology of the Mojave Desert region California, chap. 2 in Jahns, R.H., Baily T.L., eds., Geology of southern California: California Department of Mines and Geology Bulletin 170, chap. 2, p. 5.

Hinze, W.J., Aiken, C., Brozena, J., Coakley, B., Dater, D., Flanagan, G., Forsberg, R., Hildenbrand, T., Keller, G.R., Kellogg, J., Kucks, R., Lee, X., Mainville, A., Morin, R., Pilkington, M., Plouff, D., Ravat, D., Roman, D., UrrutiaFucugauchi, J., Véronneau, M., Webring, M., and Winester, D., 2005, New standards for reducing gravity data-The North American gravity database: Geophysics, v. 70, no. 4, p. J25-J32.

Izbicki, J.A., Martin, Peter, and Michel, R.L., 1995, Source, movement and age of groundwater in the upper part of the Mojave River basin, California, U.S.A., in Adar, E.M., and Leibundgut, Christian, eds., Application of tracers in arid zone hydrology: International Association of Hydrological Sciences, no. 232, p. 43-56.

International Union of Geodesy and Geophysics, 1971, Geodetic Reference System 1967: International Association of Geodesy Special Publication, no. 3, 116 p.
Jachens, R.C., Langenheim, V.E., and Christensen, A.H., 2014, Gravity analysis for groundwater basin definition-Western Mojave Desert, California, appendix 1 in Groundwater-flow and land-subsidence model of Antelope Valley, California: U.S Geological Survey Scientific Investigations Report 2014-5166, 136 p., http://dx.doi.org/10.3133/sir20145166.

Jachens, R.C., and Moring, B.C., 1990, Maps of the thickness of Cenozoic deposits and the isostatic residual gravity over basement for Nevada: U.S. Geological Survey Open-File Report 90-404, 15 p., 2 plates.

Jennings, C.W., Strand, R.G., and Rogers, T.H., 1977, Geologic map of California: California Division of Mines and Geology, scale 1:750,000.

Johnson, H.R., 1911, Water resources of Antelope Valley, California: U.S. Geological Survey Water-Supply Paper 278, $92 \mathrm{p}$.

Karki, P., Kivioja, L., and Heiskanen, W.A., 1961, Topographic isostatic reduction maps for the world for the Hayford zones 18-1, Airy-Heiskanen System, T=30 $\mathrm{km}$ : Isostatic Institute of the International Association of Geodesy, no. 35, 5 p., 20 plates.

LaCoste and Romberg, 2003, Aliod100 upgrade user's manual (revision 2.1): Austin, Tex., Exploration Instruments, 16 p., accessed at http://www.expins.com/p/pdf/104010.pdf.

Langenheim, V.E., Jachens, R.C., Matti, J.C., Hauksson, Egill, Morton, D.M., and Christensen, A., 2005, Geophysical evidence for wedging in the San Gorgonio Pass structural knot, southern San Andreas fault zone, southern California: Geological Society of America Bulletin, v. 117, no. 11, p. 1554-1572.

Leighton, D.A., and Phillips, S.P., 2003, Simulation of groundwater flow and land subsidence in the Antelope Valley Ground-Water Basin, California: U.S. Geological Survey Water-Resources Investigations Report 03-4016, 107 p.

Londquist, C.J., and Martin, Peter, 1991, Geohydrology and ground-water-flow simulation of the Surprise Spring Basin aquifer system, San Bernardino County, California: U.S. Geological Survey Water-Resources Investigations Report 89-4099, $41 \mathrm{p}$.

Mabey, D.R., 1960, Gravity survey of the western Mojave Desert, California: U.S. Geological Survey Professional Paper 316-D, p. 51-73. 
Marston, R.A., and Marston, B.K., 2017, Mountain hydrology, in Richardson, Douglas, Castree, N., Goodchild, M.F., Kobayashi, Audrey, Liu, Weidong, and Marston, R.A., eds., The international encyclopedia of geography: John Wiley \& Sons, 9 p., doi:10.1002/9781118786352.wbieg1137.

Martin, Peter, ed., with contributions by Brandt, Justin, Catchings, R.D., Christensen, A.H., Flint, A.L., Gandhok, Gini, Goldman, M.R., Halford, K.J., Langenheim, V.E., Martin, Peter, Rymer, M.J., Schroeder, R.A., Smith, G.A., and Sneed, Michelle, 2011, The source, discharge, and chemical characteristics of water from Agua Caliente Spring, Palm Springs, California: U.S. Geological Survey Scientific Investigations Report 2011-5156, 106 p.

Matti, J.C., and Morton, D.M., 1993, Paleogeographic evolution of the San Andreas Fault in southern California-A reconstruction based on a new cross-fault correlation, in Powell, R.E., Weldon, R.J., II, and Matti, J.C., eds., The San Andreas Fault System-Displacement, palinspastic reconstruction, and geologic evolution: Geological Society of America Memoir 178, p. 107-159.

Meisling, K.E., and Weldon, R.J., 1989, Late Cenozoic tectonics of the northwestern San Bernardino Mountains, southern California: Geological Society of America Bulletin, v. 101, p. $106-128$.

Mojave Basin Area Watermaster, 2015, Twenty-first annual report of the Mojave Basin Area Watermaster water year 2013-14: Mojave Water Agency web page, 189 p., accessed July 23, 2015, at http://www.mojavewater.org/downloads. html\#AnnualReports.

Morelli, C., 1974, The International Gravity Standardization Net, 1971: International Association of Geodesy Special Publication no. 4, 194 p.

National Geodetic Survey, 2011, Online Positioning User Service (OPUS): National Oceanic and Atmospheric Administration, accessed March 17, 2011, at http://www.ngs.noaa.gov/OPUS/.

Plouff, D., 1977, Preliminary documentation for a FORTRAN program to compute gravity terrain corrections based on topography digitized on a geographic grid: U.S. Geological Survey Open-File Report 77-535, 45 p.

Riverside County Superior Court, 1996, Judgment after trial, Mojave Basin Area adjudication: City of Barstow, et al. v. City of Adelanto, et al., case no. 208568, 140 p.

Robbins, S.L., and Oliver, H.W., 1970, On making inner-zone terrain corrections to gravity data: U.S. Geological Survey Internal Report, 13 p., 2 plates.
Roberts, C.W., and Jachens, R.C., 1986, High-precision gravity stations for monitoring vertical crustal motion in Southern California: U.S. Geological Survey Open-File Report $86-44,78 \mathrm{p}$.

Roberts, C.W., and Jachens, R.C., 1999, Preliminary aeromagnetic anomaly map of California: U.S. Geological Survey Open-File Report 99-440, 14 p.

Roberts, C.W., Jachens, R.C., and Oliver, H.W., 1990, Isostatic residual gravity map of California and offshore southern California: California Division of Mines and Geology California Geologic Data Map Series Map 7, scale 1:750,000.

Rydlund, P.H., Jr., and Densmore, B.K., 2012, Methods of practice and guidelines for using survey-grade global navigation satellite systems (GNSS) to establish vertical datum in the United States Geological Survey: U.S. Geological Survey Techniques and Methods, book 11, chap. D1, 102 p. with appendixes.

Saltus, R.W., and Jachens, R.C., 1995, Gravity and basin depth maps for the Basin and Range Province, western United States: U.S. Geological Survey Geophysical Map GP-1012, scale 1:2,500,000.

Siade, A.J., Nishikawa, Tracy, Rewis, D.L., and Martin, Peter, 2014, Groundwater-flow and land-subsidence model of Antelope Valley, California: U.S Geological Survey Scientific Investigation Report 2014-5166, 136 p., http://dx.doi.org/10.3133/sir20145166.

Stamos, C.L, Martin, Peter, Nishikawa, Tracy, and Cox, B.F., 2001, Simulation of ground-water flow in the Mojave River Basin, California: U. S. Geological Survey WaterResources Investigations Report 01-4002, 129 p., accessed at http://pubs.usgs.gov/wri/wri014002.

Stamos, C.L., and Predmore, S.K., 1995, Data and water-table map of the Mojave River ground-water basin, San Bernardino County, California, November 1992: U.S. Geological Survey Water-Resources Investigations Report 95-4148, 1 plate.

Subsurface Surveys, Inc., 1990, Inventory of groundwater stored in the Mojave River: Consultant's report prepared for and in the files of Mojave Water Agency, $47 \mathrm{p}$.

Superior Court of the State of California, County of Los Angeles-Central District, 2015, Judicial Council Coordination Proceeding no. 4408, Santa Clara case no. 1-05-CV-049053: Antelope Valley-East Kern Water Agency, 4 p., accessed June 5, 2017, at http://www.avek.org/fileLibrary/file_658.pdf. 
Teague, N.F., Stamos, C.L., House, S.F., and Clark, D.A., 2014, Regional water table (2012) in the Mojave River and Morongo groundwater basin, Southwestern Mojave desert, California: U.S. Geological Survey web page, accessed at http://dx.doi.org/10.5066/F7CJ8BHF.

Telford, W.M., Geldart, L.P., and Sheriff, R.E., 1990, Applied Geophysics (2nd ed.): New York, Cambridge University Press, $770 \mathrm{p}$.

Thompson, D.G., 1929, The Mohave Desert region, California-A geographic, geologic, and hydrologic reconnaissance: U.S. Geological Survey Water-Supply Paper 578, $759 \mathrm{p}$.

U.S. Census Bureau, 2017, State and county QuickFacts, population data: U.S. Census Bureau Web service, accessed April 22, 2017, at http://www.census.gov/quickfacts/table/PST045215/00.

U.S. Geological Survey, 2009, National Elevation Dataset ( $2 \mathrm{~d}$ ed.): The National Map, accessed June 8, 2017, at http://nationalmap.gov.

Ward, A.W., Dixon, G.L., and Jachens, R.C., 1993, Geologic setting of the East Antelope Basin, with emphasis on fissuring on Rogers Lake, Edwards AFB, Mojave Desert, California: U.S. Geological Survey Open-File Report 93-263, 9 p.

Western Regional Climate Center, 2015a, Period of record climate summary, Palmdale AP, California (046627), 1934-2015 Western Regional Climate Center web page, accessed July 21, 2015, at http://www.wrcc.dri.edu/cgi-bin/cliMAIN.pl?ca6627.

Western Regional Climate Center, 2015b, Period of record climate summary, El Mirage, California (042771), 1981-2010: Western Regional Climate Center web page, accessed August 2015, at http://www.wrcc.dri.edu/cgi-bin/cliMAIN.pl?ca2771. 
Prepared by the Sacramento Publishing Service Center.

For more information concerning this report, contact:

Director

U.S. Geological Survey

California Water Science Center

6000 J Street, Placer Hall

Sacramento, CA 95829

or visit our Web site at:

http://ca.water.usgs.gov 


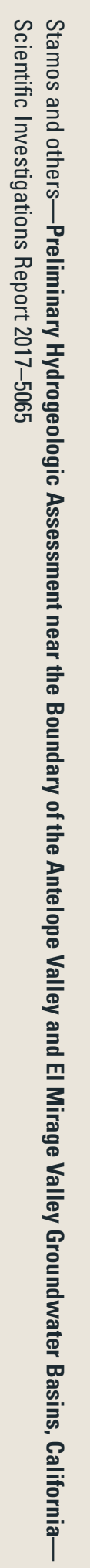

